\title{
Types and Amounts of Complementary Foods and Beverages Consumed and Developmental Milestones: A Systematic review
}

The Pregnancy and Birth to 24 Months Project

Published date: April 15 2019

Nutrition Evidence Systematic Review Center for Nutrition Policy and Promotion

Food and Nutrition Service

U.S. Department of Agriculture

3101 Park Center Drive

Alexandria, Virginia 
This systematic review was conducted by the Nutrition Evidence Systematic Review (NESR) team at the Center for Nutrition Policy and Promotion, Food and Nutrition Service, USDA. This systematic review was completed for the Pregnancy and Birth to 24 Months Project (P/B-24 Project). All systematic reviews from the P/B-24 Project are available on the NESR website: https://nesr.usda.gov

Conclusion statements drawn as part of this systematic review describes the state of science related to the specific question examined. Conclusion statements do not draw implications, nor should they be interpreted to be dietary guidance.

The contents of this document may be used and reprinted without permission. Endorsement by NESR, the Center for Nutrition Policy and Promotion, the Food and Nutrition Service, or the U.S. Department of Agriculture of derivative products developed from this work may not be stated or implied.

In accordance with Federal civil rights law and U.S. Department of Agriculture (USDA) civil rights regulations and policies, the USDA, its Agencies, offices, and employees, and institutions participating in or administering USDA programs are prohibited from discriminating based on race, color, national origin, religion, sex, gender identity (including gender expression), sexual orientation, disability, age, marital status, family/parental status, income derived from a public assistance program, political beliefs, or reprisal or retaliation for prior civil rights activity, in any program or activity conducted or funded by USDA (not all bases apply to all programs). Remedies and complaint filing deadlines vary by program or incident.

Persons with disabilities who require alternative means of communication for program information (e.g., Braille, large print, audiotape, American Sign Language, etc.) should contact the responsible Agency or USDA's TARGET Center at (202) 720-2600 (voice and TTY) or contact USDA through the Federal Relay Service at (800) 877-8339. Additionally, program information may be made available in languages other than English.

To file a program discrimination complaint, complete the USDA Program Discrimination Complaint Form, AD3027, found online at How to File a Program Discrimination Complaint and at any USDA office or write a letter addressed to USDA and provide in the letter all of the information requested in the form. To request a copy of the complaint form, call (866) 632-9992. Submit your completed form or letter to USDA by: (1) mail: U.S. Department of Agriculture, Office of the Assistant Secretary for Civil Rights, 1400 Independence Avenue, SW, Washington, D.C. 20250-9410; (2) fax: (202) 690-7442; or (3) email: program.intake@usda.gov.

USDA is an equal opportunity provider, employer, and lender.

Suggested citation for this systematic review: Nutrition Evidence Systematic Review Team and Complementary Feeding Technical Expert Collaborative. Types and Amounts of Complementary Foods and Beverages Consumed and Developmental Milestones: A Systematic Review. Pregnancy and Birth to 24 Months Project. Alexandria, VA: U.S. Department of Agriculture, Food and Nutrition Service, Center for Nutrition Policy and Promotion, February 2019. Available at: https://nesr.usda.gov/project-specific-overviewpb-24-0

This systematic review has also been published in the American Journal of Clinical Nutrition: English LK, Obbagy JO, Wong YP, Butte NF, Dewey KG, Fox MK, Greer FR, Krebs NK, Scanlon KS, Stoody EE. Complementary feeding and developmental milestones: a systematic review. Am J Clin Nutr 2019, 109(7): 879S-889S. doi.org/10.1093/ajcn/nqy321

\section{Related citations:}

- P/B-24 Project overview: Stoody EE, Spahn JM, Casavale KO. The Pregnancy and Birth to 24 Months Project: a series of systematic reviews on diet and health. Am J Clin Nutr. 2019;109(7):685S-697S. doi: 10.1093/ajcn/nay372

- P/B-24 systematic review methodology: Obbagy JE, Spahn JS, Psota TL, Spill MK, Dreibelbis C, Gungor DE, Nadaud PN, Raghavan R, Callahan EH, English LK, Kingshipp BJ, Lapergola CC, Shapiro MJ, Stoody EE. Systematic review methodology used in the Pregnancy and Birth to 24 Months Project. Am J Clin Nutr 2019;109(7):698S-704S. doi: 10.1093/ajcn/nqy226 


\section{ACKNOWLEDGEMENTS}

\section{Technical Expert Collaborative (TEC) members:}

- Nancy F. Butte, PhD, RD, USDA/ARS Children's Nutrition Research Center, Baylor College of Medicine, Department of Pediatrics, Emeritus

- Kathryn G. Dewey, PhD, University of California, Davis, Department of Nutrition

- David M. Fleischer, MD, Children's Hospital Colorado, University of Colorado School of Medicine, Department of Pediatrics, Section of Allergy and Immunology

- Mary Kay Fox, Med, Mathematic Policy Research

- Frank R. Greer, MD, University of Wisconsin School of Medicine and Public Health, Department of Pediatrics, Emeritus

- Nancy F. Krebs, MD, MS, University of Colorado School of Medicine, Department of Pediatrics

- Kelley S. Scanlon, PhD, RD, USDA Food and Nutrition Service; formerly of the Centers for Disease Control and Prevention, Division of Nutrition, Physical Activity, and Obesity

\section{Nutrition Evidence Systematic Review (NESR) team:}

- Laural K. Englishi, PhD, Panum Group, Lead Analyst (11/2016-project completion)

- Julie E. Obbagy, PhD, RD, USDA, Analyst (05/2016-project completion)

- Yat Ping Wong, MLS, MPH, USDA, Librarian

- Tricia Psota, PhD, USDA, Analyst (07/2015-06/2016)

- Perrine Naudadi, PhD, Panum Group, Analyst (07/2015-05/2016)

- Kirsten Johnsi, MS, Panum Group, Analyst (07/2015-05/2016)

\section{Project Lead:}

- Eve Essery Stoody, PhD, USDA

\section{Federal Expert Group (FEG)-Technical Expert Collaborative (TEC) Liaisons:}

- Kelley Scanlon, PhD, RD, USDA Food and Nutrition Service; formerly of the Centers for Disease Control and Prevention, Division of Nutrition, Physical Activity, and Obesity

All TEC and NESR team members, Project leads, and FEG-TEC liaisons participated in establishing the research questions, analytic framework, and study inclusion and exclusion criteria. LKE, JEO, YPW, TLP, PN, KJ, and NT developed and conducted the literature search, screened search results, and identified studies for inclusion. LKE and JEO extracted data and assessed risk of bias for included studies. NFC, KGD, DMF, MKF, FRG, NFK, and KSS reviewed and provided substantive feedback on all systematic review materials, including the synthesis of the body of evidence, conclusion statement, and grade of the strength of the evidence. LKE prepared this report and

i Under contract with the Food and Nutrition Service, United States Department of Agriculture. 
EES provided oversight. All authors critically reviewed and approved the final report. The authors declare no conflicts of interest.

FUNDING SOURCE: United States Department of Agriculture, Food and Nutrition Service, Center for Nutrition Policy and Promotion, Alexandria, VA 


\section{TABLE OF CONTENTS}

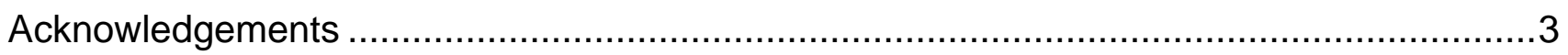

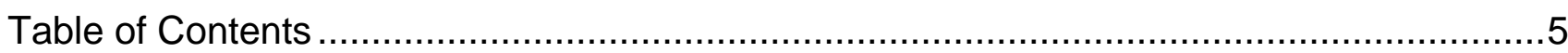

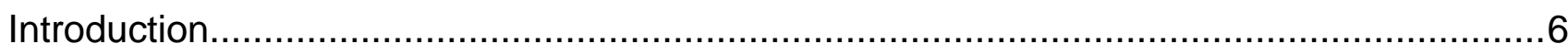

What is the relationship between the types and amounts of complementary foods and

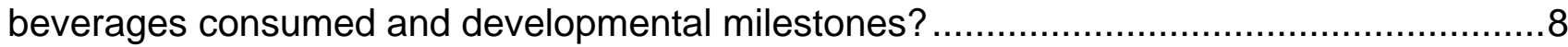

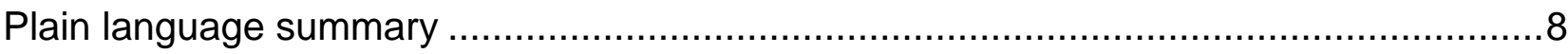

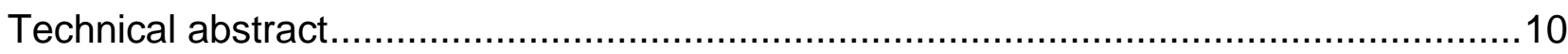

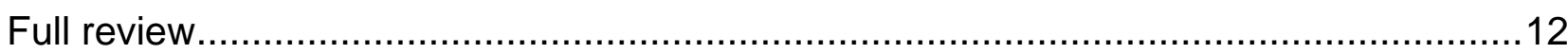

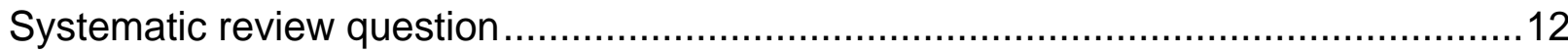

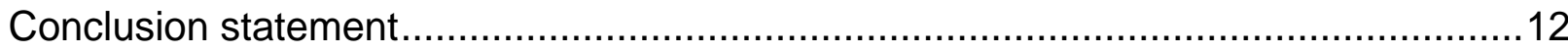

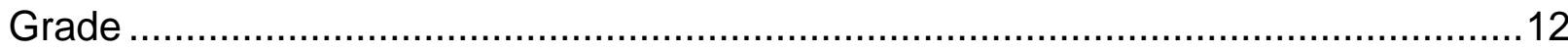

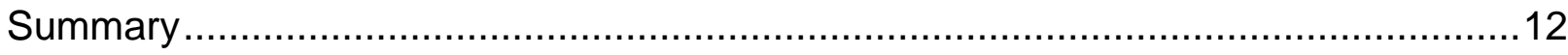

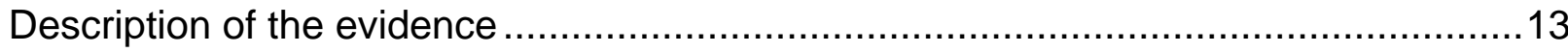

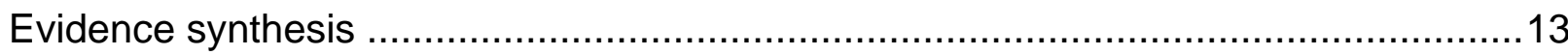

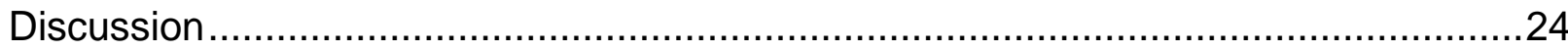

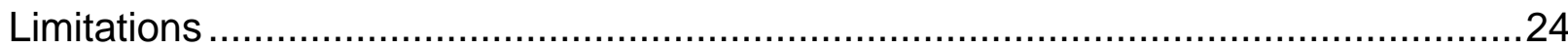

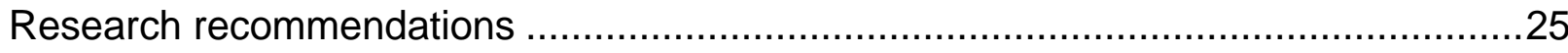

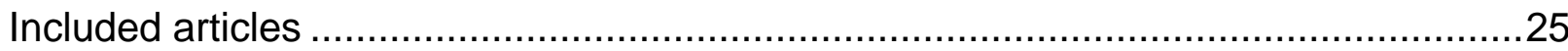

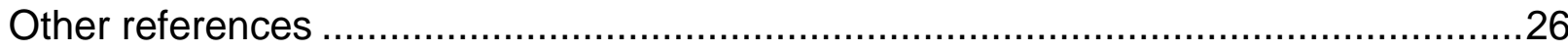

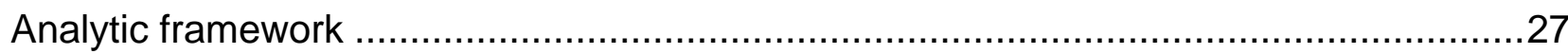

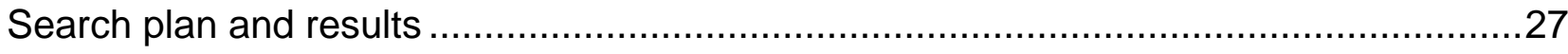

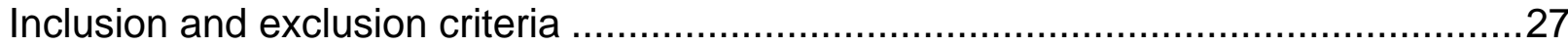

Search terms and electronic databases used ............................................................

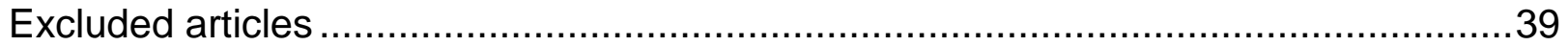

Table 1. Studies that examined dietary patterns during the complementary feeding period and developmental milestones.

Table 2. Studies that examined types and amounts of CFB consumed and developmental

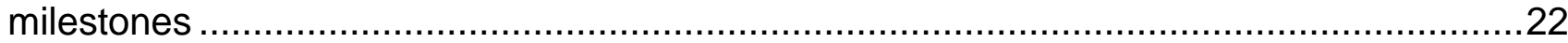

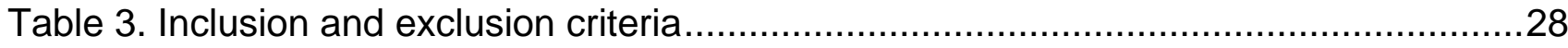

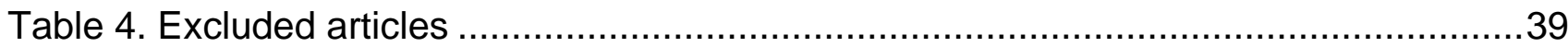

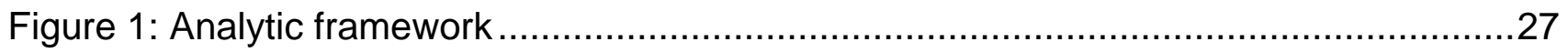

Figure 2: Flow chart of literature search and screening results ......................................38 
This document describes a systematic review conducted to answer the following question: What is the relationship between types and amounts of complementary foods and beverages consumed and developmental milestones?. This systematic review was conducted as part of the Pregnancy and Birth to 24 Months Project (P/B-24 Project) by USDA's Nutrition Evidence Systematic Review (NESR).

The purpose of the P/B-24 Project was to conduct a series of systematic reviews on diet and health for women who are pregnant and for infants and toddlers from birth to 24 months of age. This project was a joint initiative led by USDA and HHS, and USDA's NESR carried out all of the systematic reviews. A Federal Expert Group (FEG), a broadly representative group of Federal researchers and program leaders, also provided input throughout the P/B-24 Project. More information about the P/B-24 Project has been publishedii and is available on the NESR website: https://nesr.usda.gov/project-specificoverview-pb-24-0.

NESR, formerly the Nutrition Evidence Library (NEL), specializes in conducting food- and nutrition-related systematic reviews using a rigorous, protocol-driven methodology. To conduct each P/B-24 systematic review, NESR's staff worked with a Technical Expert Collaborative (TEC), which is a group of 7-8 leading subject matter experts.

NESR's systematic review methodology involves developing and prioritizing systematic review questions, searching for and selecting studies, extracting and assessing the risk of bias of data from each included study, synthesizing the evidence, developing a conclusion statement, grading the evidence underlying the conclusion statement, and recommending future research. A detailed description of the methodology used in conducting systematic reviews for the P/B-24 Project has been publishediii and is available on the NESR website: https://nesr.usda.gov/pb-24-project-methodology-0. In addition, starting on page 27, this document includes details about the methodology as it was applied to the systematic review described herein. An analytic framework that illustrates the overall scope of the question, including the population, the interventions and/or exposures, comparators, and outcomes of interest, is found on page 27 . In addition, the literature search plan, that was used to identify studies included in this systematic review is found on page 27.

\footnotetext{
ii Stoody EE, Spahn JM, Cassavale KO. The Pregnancy and Birth to 24 Months Project: a series of systematic reviews on diet and health. Am J Clin Nutr. 2019;109(7):685S-697S. doi:10.1093/ajcn/ngy372

iii Obbagy JE, Spahn JS, Psota TL, Spill MK, Dreibelbis C, Gungor DE, Nadaud PN, Raghavan R, Callahan EH, English LK, Kingshipp BJ, Lapergola CC, Shapiro MJ, Stoody EE. Systematic review methodology used in the Pregnancy and Birth to 24 Months Project. Am J Clin Nutr. 2019;109(7):698S704S. doi: 10.1093/ajcn/nqy226
} 


\section{List of abbreviations}

\begin{tabular}{ll}
\hline Abbreviation & Full description \\
\hline BF & Breast fed \\
\hline BSID & Bayley Scale of Infant Development \\
\hline CFB & Complementary Food and Beverage \\
\hline CFUI & Complementary feeding utility index \\
\hline EBF & Exclusively breast-fed \\
\hline FEG & Federal Expert Group \\
\hline HHS & Department of Health and Human Services \\
\hline IQ & Intelligence quotient \\
\hline MDI & Mental development index \\
\hline NEL & Nutrition Evidence Library \\
\hline NESR & Nutrition Evidence Systematic Review \\
\hline NIH & National Institutes of Health \\
\hline PDI & Psychomotor development index \\
\hline P/B-24 & Pregnancy and Birth to 24 Months Project \\
\hline TEC & Technical Expert Collaborative \\
\hline U.K. & United Kingdom \\
\hline U.S. & United States \\
\hline USDA & United States Department of Agriculture \\
\hline WISC & Wechsler Intelligence Scale for Children \\
\hline
\end{tabular}




\section{WHAT IS THE RELATIONSHIP BETWEEN THE TYPES AND AMOUNTS OF COMPLEMENTARY FOODS AND BEVERAGES CONSUMED AND DEVELOPMENTAL MILESTONES?}

\section{PLAIN LANGUAGE SUMMARY}

What is the question?

- What is the relationship between the types and amounts of complementary foods and beverages consumed and developmental milestones?

What is the answer to the question?

- There is insufficent evidence to draw a conclusion about the relationships between types and amounts of complementary foods and beverages consumed and developmental milestones.

Why was this question asked?

- This important public question was identified as part of the U.S. Department of Agriculture and Department of Health and Human Services Pregnancy and Birth to 24 Months Project

\section{How was this question answered?}

- A team of staff from the Nutrition Evidence Systematic Review conducted a systematic review in collaboration with a group of experts called a Technical Expert Collaborative.

What is the population of interest?

- Generally healthy infants and toddlers who were fed complementary foods and beverages from ages 0-24 months and had developmental milestone outcomes examined through 18 years of age.

\section{What evidence was found?}

- This review includes eight studies published since 1980

- The studies varied in terms of the types and/or amounts of complementary foods and beverages examined, which included dietary patterns consumed during the complementary feeding period, meat and/or fortified-cereal intake, and foods with differing levels of DHA or phytate

- Different types of developmental milestone outcomes were measured between 4 months and 8.5 years of age, including:

- communication development such as sentence repetition

- cognitive development such as mental development index scores

- motor development such as psychomotor development index scores

- neurological development such as cortical processing 
- There are limitations in the evidence as follows: an inadequate number of studies that were comparable in terms of design, and variation in the types of complementary foods and beverages examined, how and when developmental milestones outcomes were assessed, and reported results

\section{How up-to-date is this review?}

- This review includes literature from 01/1980 to 07/2016 


\section{TECHNICAL ABSTRACT}

\section{Background}

- Systematic reviews were conducted as part of the U.S. Department of Agriculture and Department of Health and Human Services Pregnancy and Birth to 24 Months Project.

- The goal of this systematic review was to examine the following question: What is the relationship between types and amounts of complementary foods and beverages (CFB) consumed and developmental milestones?

- Complementary feeding is the process that starts when human milk or infant formula is complemented by other foods and beverages, beginning during infancy and typically continuing to 24 months of age. CFB were defined as foods and/or beverages other than human milk or infant formula (liquids, semisolids, and solids) provided to an infant or young child to provide nutrients and energy.

\section{Conclusion Statement}

- There was insufficient evidence to draw a conclusion about the relationships between types and amounts of CFB consumed and developmental milestones.

- Grade: Grade Not Assignable

\section{Methods}

- This systematic review was conducted by a team of staff from the Nutrition Evidence Systematic Review in collaboration with a Technical Expert Collaborative.

- Literature search was conducted using 4 databases (PubMed, Cochrane, Embase, and $\mathrm{CINAHL}$ ) to identify articles that evaluated the intervention or exposure of timing of CFB introduction and the outcomes of developmental milestones. A manual search was conducted to identify articles that may not have been included in the electronic databases searched. Articles were screened by two analysts independently for inclusion based on pre-determined criteria.

- Data extraction and risk of bias assessment were conducted for each included study, and both were checked for accuracy. The body of evidence was qualitatively synthesized to inform development of a conclusion statement(s), and the strength of evidence was graded using pre-established criteria evaluating the body of evidence on risk of bias, adequacy, consistency, impact, and generalizability.

\section{Summary of the Evidence}

- This review includes eight studies published since 1980 that examined the relationship between consuming different types and/or amounts of CFB and developmental milestones during childhood through 18 years of age, including three randomized controlled trials and five prospective cohort studies.

- The studies varied in terms of the types and/or amounts of CFB examined, which included dietary patterns consumed during the complementary feeding period, meat and/or fortified-cereal intake, and foods with differing levels of DHA or phytate 
- Different types of developmental milestone outcomes were measured between 4 months and 8.5 years of age, including:

- Communication (e.g., sentence repetition)

- Cognitive (e.g., mental development index)

- Motor (e.g., psychomotor development index)

- Neurological (e.g., cortical processing)

- Three articles from two observational studies identified positive associations between dietary patterns emphasizing vegetables and meats during the complementary feeding period, and intelligence quotient (IQ) between ages 48.5y. However, a conclusion could not be drawn due to low generalizability and heterogeneity in exposures, observed effects, and potential confounding

- Because there was substantial variation in how studies were designed, it is difficult to compare and contrast the reported results.

- No conclusion regarding the relationship between types and/or amounts of CFB and developmental milestones could be drawn due to an inadequate number of studies that were comparable in terms of design, the types of CFB examined, how and when developmental milestones outcomes were assessed, and reported results. 


\section{FULL REVIEW}

\section{Systematic review question}

What is the relationship between the types and amounts of complementary foods and beverages consumed and developmental milestones?

\section{Conclusion statement}

There is insufficient evidence to draw a conclusion about the relationship between the types or amounts of complementary foods and beverages (CFB) consumed and developmental milestones.

\section{Grade}

Grade Not Assignable

\section{Summary}

- This review includes eight studies published since 1980 that examined the relationship between consuming different types and/or amounts of complementary foods or beverages (CFB) and developmental milestones during childhood through 18 years of age, including three randomized controlled trials and five prospective cohort studies.

- CFB were defined as foods and/or beverages other than human milk or infant formula (liquids, semisolids, and solids) provided to an infant or young child to provide nutrients and energy.

- The studies varied in terms of the types and/or amounts of CFB examined, which included dietary patterns consumed during the complementary feeding period, meat and/or fortified-cereal intake, and foods with differing levels of DHA or phytate

- Different types of developmental milestone outcomes were measured between 4 months and 8.5 years of age, including:

- Communication (e.g., sentence repetition)

- Cognitive (e.g., mental development index)

- Motor (e.g., psychomotor development index)

- Neurological (e.g., cortical processing)

- Three articles from two observational studies identified positive associations between dietary patterns emphasizing vegetables and meats during the complementary feeding period, and intelligence quotient (IQ) between ages 4$8.5 y$. However, a conclusion could not be drawn due to low generalizability and heterogeneity in exposures, observed effects, and potential confounding

- Because there was substantial variation in how studies were designed, it is difficult to compare and contrast the reported results.

- No conclusion regarding the relationship between types and/or amounts of CFB and developmental milestones could be drawn due to an inadequate number of studies that were comparable in terms of design, the types of CFB examined, how and when developmental milestones outcomes were assessed, and reported results 


\section{Description of the evidence}

This systematic review includes eight studies that examined the association between types or amounts of CFB and developmental milestones, including three RCTs (1-3) and five prospective cohort studies (4-8).

According to the Human Development Index (9), all of the included studies were conducted in countries that were identified as "very high" (U.K., U.S., Sweden, and Netherlands). Most of the studies included participants who were full term (gestational age $>37$ wk) and excluded participants with illnesses or conditions that could impact complementary feeding or development. All of the prospective cohort studies included subjects who had a normal birth weight $(>2.5 \mathrm{~kg})$. Two studies specified that their samples were majority white $(4,8)$, and the remaining studies did not specify the racial/ethnic make-up of subjects.

The studies in this review examined the following interventions/exposures: dietary patterns $(4-6,8)$ meat compared to cereal $(2)$; amount of meat consumed $(7)$; phytate-reduced milkcereal-drink and porridge (3); and DHA-enriched compared to control baby-food (1). A variety of validated, developmental indices/scales were used to examine outcomes ranging in age from birth to 11.5y, including the Bayley Scales of Infant Development (BSID), the Wechsler Intelligence Scale for Children (WISC), The Denver Developmental Screening Test, and the Developmental Neuropsychological Assessment (NEPSY). Developmental milestone outcomes that were reported by these studies fit within one or more of the following domains (10): communication $(4,5)$, cognitive $(2,3,5-8)$, motor $(2-5,7)$, or other neurological (1), or behavioral $(\underline{15})$ development. No studies were identified that reported the association between types or amounts of CFB introduced and social/emotional development outcomes, executive function, or incidence and prevalence of anxiety or depression. Table 1 describes the studies and reported results from included studies that examined dietary patterns and developmental milestone outcomes. Table 2 describes the studies and reported results from included studies that examined specific types or amounts of CFB and developmental milestone outcomes.

\section{Evidence synthesis}

\section{Dietary patterns and developmental milestones}

\section{Dietary patterns and communication development}

Dagnelie and van Staveren (4) reported that infants who consumed a 'Macrobiotic' dietary pattern from 4-18 mo of age had significantly slower language development, though age of assessment was not reported. Gale et al. (5) reported no significant associations between dietary pattern scores at either 6 or 12 mo of age and language development at 4 $\mathrm{y}$ in adjusted models, though analyses did not adjust for education or race/ethnicity of participants. Gale et al. (5) adjusted for several baseline characteristics but Dagnelie and van Staveren (4) did not account for differences between groups in birth weight and BF duration. Notably in that study (4), there were also several concurrent factors, such as relatively high rates of wasting and rickets in the 'Macrobiotic' relative to 'Omnivorous' groups and may have impacted development. The magnitude of differences in that study was notable and macrobiotic diets tend to be deficient in several nutrients that could affect development. However, prenatal differences and human milk nutrient content cannot be ruled out as factors. Therefore, differences in outcome assessment, lack of adjustment for confounding factors, limited generalizability, and inconsistency in findings limit the ability to 
draw a conclusion about the relationship between dietary patterns during the complementary feeding period and communication development.

\section{Dietary patterns and motor development}

Dagnelie and van Staveren (4) found slower gross-motor development in infants with a 'Macrobiotic' compared to 'Omnivorous' diet pattern (see Table 1), but no differences in terms of fine-motor development. Gale et al. (5) reported no significant associations between dietary pattern scores at either 6 or $12 \mathrm{mo}$ and visuomotor precision at $4 \mathrm{y}$. These studies were difficult to summarize because of the variation in dietary patterns and types of motor development outcomes examined.

\section{Dietary patterns and cognitive development}

Three prospective cohort studies examined the relationship between dietary patterns and cognitive milestones $(5,6,8)$. Table 2 describes information regarding CFB included in each dietary pattern and study results in more detail. All three studies reported that dietary patterns emphasizing vegetables and meat at $6 \mathrm{mo}$ of age were associated with better verbal IQ scores in childhood at $~ 4-8.5 \mathrm{y}$, compared to other dietary patterns (Table 2). Gale et al. (5) reported that scores on the U.K. 'Infant guidelines' pattern scores at age 6 mo (i.e., higher scores reflect higher adherence to complementary feeding guidelines in the U.K.) were positively associated with full-scale and verbal IQ scores at $4 \mathrm{y}$, and at age 12 mo were positively associated with full-scale IQ scores at $4 \mathrm{y}$. No significant associations were reported between 'Adult Foods' pattern scores at age 6 or 12 mo and IQ at 4 y. In terms of cognitive outcomes aside from IQ, Gale et al. (5) reported no significant associations between either dietary pattern and visual form constancy or visual attention at 4 y. Golley et al. (6) reported that scores on the complementary feeding utility index (CFUI) at age 6 mo (i.e., higher scores reflect healthier patterns based on complementary feeding recommendations in the U.K.) were positively associated with full-scale, verbal, and performance IQ scores at age $8.5 \mathrm{y}$.

Smithers et al. (8) reported significant positive associations between the following dietary pattern scores and IQ scores at 8 y (see Table 1 for full results):

- 'Homemade traditional' (e.g., potatoes/vegetables, meat) at age 6 mo and full-scale, verbal, and performance IQ;

- 'Contemporary foods' (e.g., legumes, fruits, and/or vegetables, nuts, egg) at age 15 and $24 \mathrm{mo}$ and full-scale and verbal IQ;

- 'Ready-to-eat foods' (e.g., biscuits, bread/toast, breakfast cereal, yogurt, milk pudding, cola) at age $24 \mathrm{mo}$ and full-scale and verbal IQ.

Significant negative associations were identified between the following dietary pattern scores and IQ scores at 8y (see Table 1 for full results):

- 'Discretionary foods' (e.g., biscuits, crisps, sweets, chocolate, cola) at ages 6 mo and full-scale, verbal, and performance IQ, 'Discretionary foods' at $15 \mathrm{mo}$ and fullscale and verbal IQ, and 'Discretionary Foods' at 24 mo and full-scale IQ.

- 'Ready-made' (e.g., commercial manufactured foods including rice/baby cereal, meat from a tin/jar, vegetables, fruit puddings, milk puddings, and fish) at $6 \mathrm{mo}$ and full-scale and verbal IQ.

- 'Ready-made' (e.g., commercial manufactured foods) at 15 mo and full-scale, verbal, and performance IQ.

Smithers et al. (8) acknowledged that all observed, significant differences were less than 
one IQ point and results were primarily driven by the verbal IQ subscale.

Although all three studies analyzed scores on dietary patterns that were related to higher intake of vegetables and meat/meat products at $6 \mathrm{mo}$, dietary patterns were analyzed heterogeneously across studies and may be less generalizable to U.S. patterns. For example, Golley et al. (6) analyzed CFUI scores, which were reflective of better adherence to broad complementary feeding recommendations in Australia, New Zealand, North America, and the U.K. from 2003-2010. Gale et al. (5) analyzed scores on the Infant Guidelines pattern, which was reflective of better adherence to the infant feeding manual from the U.K. Department of Health in 2006. However, the patterns analyzed in Smithers et al. (8) have previously shown both similarity and discordance with U.S. patterns, see (11). Some of the patterns identified in Smithers et al. (8) included foods such as gravy/soy sauce, were only observed at 15 or 24 mo, or were not well described. In addition, the dietary patterns from Smithers et al. (8) did not clearly align with the three dietary patterns that were used to obtain CFUI scores in Golley et al. (6), despite data being from the same cohort. Among the three studies, dietary patterns characterized by foods and/or food groups divergent from complementary feeding recommendations, such as bread, biscuits, and fizzy drinks/cola, had either negative or null associations with IQ in early-mid childhood. All three cohort studies controlled for the majority, if not all, potential key confounders. However, the interval between assessment of dietary patterns during the complementary feeding period and outcome assessment of IQ varied across studies, with dietary patterns as early as 6 mo of age and outcome assessments from 4-8.5 y of age. It is plausible that uncontrolled factors during this interval may confound the relationship between dietary patterns consumed during the complementary feeding period and developmental milestones. 
Table 1. Studies that examined dietary patterns during the complementary feeding period and developmental milestones.

\begin{tabular}{|c|c|c|c|c|c|}
\hline $\begin{array}{l}\text { Reference, } \\
\text { design, } \\
\text { country, } \\
\text { sample size }\end{array}$ & $\begin{array}{l}\text { Outcome } \\
\text { assessment }{ }^{1}\end{array}$ & Independent variable or Exposure ${ }^{2}$ & Cognitive results & Motor results & $\begin{array}{l}\text { Communication } \\
\text { results }\end{array}$ \\
\hline $\begin{array}{l}\text { Dagnelie and } \\
\text { van Staveren, } \\
1994(4) \\
\text { Prospective } \\
\text { Cohort Study, } \\
\text { Netherlands } \\
\text { N: } 106\end{array}$ & $\begin{array}{l}\text { Observation and } \\
\text { maternal-report } \\
\text { via Denver } \\
\text { Developmental } \\
\text { Screening Test at } \\
\text { ages 14-18mo }\end{array}$ & $\begin{array}{l}\text { 'Macrobiotic' pattern (at } \sim 4-10 \mathrm{mo:} \\
\text { unpolished rice, pulses, vegetables, } \\
\text { seaweeds, fermented foods, nuts, } \\
\text { seeds, and seasoned foods; no } \\
\text { vitamin D supplements, animal } \\
\text { products, fats/oils, and fish) vs. } \\
\text { 'Omnivorous' pattern (not described) }\end{array}$ & & $\begin{array}{l}\text { Sitting/head balance: } \\
\text { SDS=-0.48, 95\% Cl: - } \\
\text { 0.94, -0.02, } P=0.04 ; \\
\text { Locomotion: SDS=-0.60, } \\
95 \% \mathrm{Cl}:-0.97,-0.24 ; \\
\mathrm{P}=0.001 \text {; } \\
\text { Gross motor: SDS=-0.63, } \\
95 \% \mathrm{Cl}:-1.00,-0.26 ; \\
\mathrm{P}<0.001 \\
\text { Fine motor (NSA) }\end{array}$ & $\begin{array}{l}\text { Speech: SDS=- } \\
0.42,95 \% \mathrm{Cl}:-0.84 \\
-0.05 ; P=0.03\end{array}$ \\
\hline \multirow[t]{4}{*}{$\begin{array}{l}\text { Gale et al., } \\
2009 \text { (5) } \\
\text { Prospective } \\
\text { Cohort Study, } \\
\text { U.K. } \\
\text { N: } 241\end{array}$} & $\begin{array}{l}\text { IQ via WISC; } \\
\text { Developmental } \\
\text { Neuropsychologic } \\
\text { al Assessment } \\
\text { and Test of } \\
\text { Visual-Perceptual } \\
\text { Skills at age } 4 y\end{array}$ & $\begin{array}{l}\text { 'Infant guidelines' pattern at } 6 \mathrm{mo} \text { : } \\
\text { high fruit, vegetables, meat and fish, } \\
\text { home-prepared foods, breast milk; } \\
\text { low commercial baby foods, and } \\
\text { formula }\end{array}$ & $\begin{array}{l}\text { Full-scale } \beta=0.18 \\
95 \% \mathrm{Cl}: 0.04,0.31 \\
\mathrm{P}<0.01 ; \text { Verbal } \\
\beta=0.14 ; 95 \% \mathrm{Cl}: 0.01 \\
0.27, \mathrm{P}<0.05 \\
\text { Performance (NSA) } \\
\text { IQ at } 4 \mathrm{y}\end{array}$ & $\begin{array}{l}\text { Visuomotor precision at } \\
4 y(N S A)\end{array}$ & $\begin{array}{l}\text { Sentence repetition } \\
\text { or verbal fluency at } \\
4 y(N S A)\end{array}$ \\
\hline & & & $\begin{array}{l}\text { Visual form constancy } \\
\text { or visual attention at } \\
4 y(N S A)\end{array}$ & & \\
\hline & & $\begin{array}{l}\text { 'Infant guidelines' pattern at } 12 \mathrm{mo} \text { : } \\
\text { high vegetables, fruit, and home- } \\
\text { prepared foods }\end{array}$ & $\begin{array}{l}\text { Full-scale: } \beta=0.18 ; \\
95 \% \mathrm{Cl}: 0.04,0.31 \\
\mathrm{P}<0.01 ; \text { Verbal (NSA); } \\
\text { Performance (NSA) } \\
\text { IQ at } 4 \mathrm{y}\end{array}$ & $\begin{array}{l}\text { Visuomotor precision at } \\
4 y(N S A)\end{array}$ & $\begin{array}{l}\text { Sentence repetition } \\
\text { or verbal fluency at } \\
4 y(N S A)\end{array}$ \\
\hline & & & Visual form constancy & & \\
\hline
\end{tabular}




\begin{tabular}{|c|c|c|c|}
\hline & $\begin{array}{l}\text { or visual attention at } \\
4 y(N S A)\end{array}$ & & \\
\hline \multirow{2}{*}{$\begin{array}{l}\text { 'Adult foods' pattern at } 6 \mathrm{mo} \text { : high } \\
\text { bread, savory snacks, biscuits, chips, } \\
\text { squash, cereals; low breast milk and } \\
\text { baby rice }\end{array}$} & $\begin{array}{l}\text { Full-scale, Verbal, } \\
\text { Performance IQ at } 4 y \\
\text { (NSA) }\end{array}$ & $\begin{array}{l}\text { Visuomotor precision at } \\
4 y(N S A)\end{array}$ & $\begin{array}{l}\text { Sentence repetition } \\
\text { or verbal fluency at } \\
4 y(N S A)\end{array}$ \\
\hline & $\begin{array}{l}\text { Visual form constancy } \\
\text { or visual attention at } \\
4 y(N S A)\end{array}$ & & \\
\hline \multirow[t]{2}{*}{$\begin{array}{l}\text { 'Adult foods' pattern at } 12 \mathrm{mo} \text { : high } \\
\text { crisps, savory snacks, processed } \\
\text { meat, squash and chips }\end{array}$} & $\begin{array}{l}\text { Full-scale, Verbal, } \\
\text { Performance IQ at 4y } \\
\text { (NSA) }\end{array}$ & $\begin{array}{l}\text { Visuomotor precision at } \\
4 y(N S A)\end{array}$ & $\begin{array}{l}\text { Sentence repetition } \\
\text { or verbal fluency at } \\
4 y(N S A)\end{array}$ \\
\hline & $\begin{array}{l}\text { Visual form constancy } \\
\text { or visual attention at } \\
4 y \text { (NSA) }\end{array}$ & & \\
\hline
\end{tabular}

\begin{tabular}{lll}
\hline Golley et al., & IQ via WISC-III at & CFUI scores at age 6mo: derived and \\
$2013(6)$ & age $\sim 8.5 y$ & analyzed as a continuous variable \\
Prospective & & from 14 components: BF duration, \\
Cohort Study, & age of introduction to solids, textured \\
U.K. & foods, and minimizing ready-made \\
N: 7170 & infant foods
\end{tabular}

Full-scale $\beta=1.92$;

95\% Cl: 1.38, 2.47,

$P<0.001$; Verbal

$\beta=1.92 ; 95 \% \mathrm{Cl}: 1.37$,

2.48, $P<0.001$;

Performance IQ

$\beta=1.33 ; 95 \% \mathrm{Cl}: 0.74$,

1.92, $P<0.001$ at

$\sim 8.5 \mathrm{y}$

\begin{tabular}{|c|c|c|c|}
\hline \multirow[t]{2}{*}{$\begin{array}{l}\text { Smithers et al., } \\
2012 \text { (8) } \\
\text { Prospective } \\
\text { Cohort Study, } \\
\text { U.K. } \\
\text { N: } 7097\end{array}$} & $\begin{array}{l}\text { IQ via WISC-III at } \\
\text { age } \sim 8.5 y\end{array}$ & $\begin{array}{l}\text { 'Homemade traditional' pattern at } \\
6 \text { mo: home-prepared meats, } \\
\text { vegetables, potato, fish, fruit } \\
\text { puddings, and milk puddings }\end{array}$ & $\begin{array}{l}\text { Full-Scale } \beta=0.69 ; 95 \% \\
\text { Cl:0.18,1.21, } P=0.009 \\
\text { Verbal } \beta=0.59 ; 95 \% \mathrm{Cl} \\
\text { 0.04,1.15, } P=0.037 \\
\text { Performance IQ } \beta=0.63 \\
95 \% \mathrm{Cl}: 0.13,1.13, P \\
=0.014 ; \text { at } ~ 8.5 \mathrm{y}\end{array}$ \\
\hline & & $\begin{array}{l}\text { 'Homemade traditional' pattern at } \\
\text { 15mo: home-prepared meats, }\end{array}$ & $\begin{array}{l}\text { Full-Scale, Verbal, or } \\
\text { Performance IQ at }\end{array}$ \\
\hline
\end{tabular}


'Homemade traditional' pattern at 24mo: potatoes, other vegetables,

Full-Scale, Verbal, or meat products, gravy/soy sauce,

Performance IQ at

green beans

'Discretionary foods' pattern at $6 \mathrm{mo}$ : crisps, sweets, chocolate, cola, biscuits

Full-Scale $\beta=-1.15$,

95\% Cl: $-1.80,-0.50$,

$P=0.001$; Verbal $\beta=-$

1.23, 95\% Cl: -1.93 , -

$0.54, P=0.001$

Performance IQ $\beta=-$

$0.77,95 \% \mathrm{Cl}:-1.42$, -

$0.12, P=0.021$ at $\sim 8.5 \mathrm{y}$

'Discretionary foods' pattern at $15 \mathrm{mo}$ : crisps, sweets, chocolate, cola,

Full-Scale $\beta=-0.86$;

95\% Cl:-1.52, -0.20

$P=0.012$; Verbal $\beta=-$

$1.20 ; 95 \% \mathrm{Cl}:-1.90$, -

$0.50, P=0.001$;

Performance IQ (NSA)

at $\sim 8.5 y$

'Discretionary foods' pattern at $24 \mathrm{mo}$ : crisps, sweets, fizzy drink, chocolate, cola, tomato ketchup, savory snacks, baked beans, biscuits

Full-Scale $\beta=-0.68$;

$95 \%$ Cl:-1.36, -0.01 ,

$P=0.046$; Verbal and

Performance IQ (NSA)

at $~ 8.5 y$

'Ready-made' pattern at $6 \mathrm{mo}$ : not

Full-Scale $\beta=-0.63$;

described; commercial manufactured

foods including rice/baby cereal,

95\% Cl:-1.06,-0.19,

$P=0.005$; Verbal $\beta=-$

tinned/jar meat, fruit puddings,

$0.84 ; 95 \% \mathrm{Cl}:-1.28$,

vegetable, milk puddings, and fish

$0.39, P<0.001$

Performance IQ (NSA)

at $\sim 8.5 \mathrm{y}$ 
'Ready-made' pattern at $15 \mathrm{mo}$ : not described; commercial baby foods that required little cooking or preparation
Full-Scale $\beta=-1.11$

95\% Cl:-1.71,-0.50;

$P<0.01$; Verbal $\beta=-$

$1.18 ; 95 \% \mathrm{Cl}:-1.78,-$

$0.59, P<0.001$;

Performance IQ at

$\sim 8.5 \mathrm{y} \beta=-0.71 ; 95 \% \mathrm{Cl}$ :

$-1.34,-0.09, P=0.026$

'Ready-to-eat foods' pattern at 24mo: Full-Scale $\beta=0.76$;

biscuits, bread/toast, breakfast $\quad 95 \% \mathrm{Cl}: 0.23,1.29$,

cereal, yogurt, milk pudding, cola $\quad P=0.005$; Verbal

$\beta=0.90 ; 95 \% \mathrm{Cl}$ :

$\mathrm{Cl}=0.36,1.44, P$

$=0.001 ;$ Performance

IQ (NSA) at $~ 8.5 y$

'Contemporary foods' pattern at 15mo: herbs, legumes, nuts, raw fruits and vegetables

Full-Scale $\beta=0.67$;

95\% Cl:0.07,1.26,

$P=0.029$; Verbal

$\beta=0.63 ; 95 \% \mathrm{Cl}$ :

$0.004,1.25, P=0.049$;

Performance IQ (NSA)

at $\sim 8.5 \mathrm{y}$

'Contemporary foods' pattern at

24mo: legumes, raw apple, other raw

fruit, herbs, cheese, apple juice,

other fruit juice, egg

Full-Scale $\beta=0.09$

95\%Cl: 0.13,1.66,

$P=0.023$; Verbal

$\beta=0.80 ; 95 \% \mathrm{Cl}$ :

$0.08,1.52, P=0.029$;

Performance IQ (NSA)

at $\sim 8.5 \mathrm{y}$

'BF' pattern at 6mo: not described; strong correlation with breastfeeding

and negative loadings for infant

Full-Scale $\beta=0.97$;

95\% Cl: 0.49, 1.45,

$P<0.001$; Verbal

$\beta=1.13,95 \% \mathrm{Cl}: 0.65$,

1.61, $P<0.001$; 
1. Developmental milestone outcomes were categorized in accordance with the analytic framework, and for these studies included the following domains: cognitive, motor, and communication development. No studies examined social/emotional development or incidence of anxiety/depression in relation to complementary feeding (also see Figure 1).

2. The independent variables/exposures included in this systematic review were types and amounts of CFB, which included studies that examined dietary patterns during the complementary feeding period described in this table.

Abbreviations: BF, breast-fed; CFB, complementary food/beverage; CFUI: Complementary feeding utility index; FF, formula-fed; acid; IQ, intelligence quotient; PCA, principal component analysis; NSA, non-significant association; NSGD, no significant group differences; RCT, randomized controlled trial; WISC, Wechsler Intelligence Scale for Children 


\section{Specific types and amounts of CFB consumed and developmental milestones}

Meat with or without cereal and cognitive and/or motor development

Two studies $(2,7)$ reported no significant association between meat consumption, starting at either 4 or $5 \mathrm{mo}$ of age, and BSID scores for cognitive and motor development (i.e., MDI or PDI scores on the BSID) (Table 2). Krebs et al. (2) additionally reported no difference between the meat relative to cereal groups in terms of behavior developmental index (BDI) scores at 7 or 12 mo. Estimated iron content of meat intake was not reported in Morgan (7). The meat (beef) in Krebs et al. (2) contained $15 \mu \mathrm{g}$ of iron per gram relative to iron-fortified, dry cereal, which contained $740 \mu \mathrm{g}$ of iron per gram. Although the cereal group had higher intake of iron, both groups in Krebs (2) had similar iron status and similar energy intakes from CFB. Morgan et al. (7) found significant positive associations, such that greater meat intake from age 4-12 mo or 4-16 mo was related to higher PDI scores at 22 mo. However, whether groups in that study were similar in baseline characteristics could not be determined and analyses were not adjusted for potential key confounding factors.

Phytate-reduced CFB and cognitive and motor development

Lind et al. (3) reported no significant differences between groups receiving reducedphytate compared to regular-phytate CFB with regard to MDI or PDI scores at ages 7, 13, or $18 \mathrm{mo}$ (Table 2).

\section{DHA-enriched CFB and neurological development}

Infants who received DHA-enriched baby food compared to control CFB from ages 6-12 mo had significantly better visual acuity at 9 and 12 mo of age, suggesting improved visual cortex development that corresponds to $\sim 1.5$ lines on an eye chart (1) (Table 2). Notably, the infants fed DHA-enriched baby food vs. control baby food had an $83 \%$ increase in DHA blood-levels, which was associated with a 2-fold greater intake of DHA from both CFB and human milk combined. 
Table 2. Studies that examined types and amounts of CFB consumed and developmental milestones

\begin{tabular}{|c|c|c|c|c|c|}
\hline $\begin{array}{l}\text { Reference, } \\
\text { study design, } \\
\text { country, } \\
\text { sample size }\end{array}$ & Outcome assessment ${ }^{1}$ & Independent variable or Exposure ${ }^{2}$ & $\begin{array}{l}\text { Cognitive } \\
\text { results }\end{array}$ & $\begin{array}{l}\text { Motor } \\
\text { results }\end{array}$ & Other results \\
\hline $\begin{array}{l}\text { Hoffman et al., } \\
2004 \text { (1) } \\
\text { RCT, U.S. } \\
\text { N: } 51\end{array}$ & $\begin{array}{l}\text { VEP swept parameter protocol } \\
\text { (visual acuity) and Infant Randot } \\
\text { Stereocards (stereoacuity) at age } 9 \\
\text { and } 12 \mathrm{mo}\end{array}$ & $\begin{array}{l}\text { DHA group (CFB enriched with DHA } \\
\text { from egg-yolk for } 6 \mathrm{mo} \text {, age } 6-12 \mathrm{mo} \text { ) } \\
\text { vs. Control group (Unenriched CFB } \\
\text { for } 6 \mathrm{mo} \text {, age } 6-12 \mathrm{mo} \text { ) }\end{array}$ & & & $\begin{array}{l}\text { Increase in VEP } \\
\text { acuity at } 9 \text { mo by } \\
0.14 \text { logMAR and } \\
\text { at } 12 \text { mo by } 0.16 \\
\text { logMAR, } P<0.002 ; \\
\text { Stereoacuity at } \\
12 \text { mo (NSGD) }\end{array}$ \\
\hline $\begin{array}{l}\text { Krebs et al., } \\
2006(2) \\
\text { RCT, U.S. } \\
\text { N: } 72\end{array}$ & BSID-II at ages 7 and $12 \mathrm{mo}$ & $\begin{array}{l}\text { Meat group (pureed beef ad libitum } \\
\text { for } 2 \mathrm{mo} \text {, age } 5-7 \mathrm{mo} \text { ) vs. Cereal } \\
\text { group (Fe-fortified infant rice cereal } \\
\text { ad libitum for } 2 \mathrm{mo} \text {, age } 5-7 \mathrm{mo} \text { ) }\end{array}$ & $\begin{array}{l}\text { MDI at } 7 \text { or } \\
12 \mathrm{mo} \text { (NSGD) }\end{array}$ & $\begin{array}{l}\text { PDI at } 7 \text { or } \\
12 \text { mo } \\
\text { (NSGD) }\end{array}$ & $\begin{array}{l}\text { BDI at } 7 \text { or } 12 \mathrm{mo} \\
\text { (NSGD) }\end{array}$ \\
\hline $\begin{array}{l}\text { Lind et al., } \\
2004(3) \\
\text { RCT, Sweden } \\
\text { N: } 194\end{array}$ & BSID at ages 7,13 , and $18 \mathrm{mo}$ & $\begin{array}{l}\text { Commercial phytate group (MCD and } \\
\text { porridge with regular phytate levels } \\
\text { for } 6 \text { mo, age } 6-12 \mathrm{mo} \text { ) vs. Reduced } \\
\text { phytate group (MCD and porridge } \\
\text { with reduced phytate levels for } 6 \mathrm{mo} \text {, } \\
\text { age } 6-12 \mathrm{mo} \text { ) vs, Infant formula group } \\
\text { (milk-based infant formula and } \\
\text { porridge for } 6 \mathrm{mo} \text {, age } 6-12 \mathrm{mo} \text { ) }\end{array}$ & $\begin{array}{l}\text { MDI at } 7,13 \text {, or } \\
18 m o(N S G D)\end{array}$ & $\begin{array}{l}\text { PDI at } 7 \\
13 \text {, or } \\
18 \mathrm{mo} \\
\text { (NSGD) }\end{array}$ & \\
\hline \multirow[t]{2}{*}{$\begin{array}{l}\text { Morgan et al., } \\
2004(7) \\
\text { Prospective } \\
\text { Cohort Study, } \\
\text { U.K. } \\
\text { N: } 144\end{array}$} & BSID-II at ages $22 \mathrm{mo}$ & $\begin{array}{l}\text { Amount of meat at } 4-12 \mathrm{mo} \text { (total } \\
\text { grams of red [beef, pork and lamb] } \\
\text { and white [chicken, turkey, fish] meat } \\
\text { consumed) }\end{array}$ & $\begin{array}{l}\text { MDI at 22mo } \\
\text { (NSA) }\end{array}$ & $\begin{array}{l}\text { PDI at } \\
22 \mathrm{mo}, \\
\beta=6.6, \\
95 \% \mathrm{Cl}: \\
2.0,11.1, \\
P=0.005\end{array}$ & \\
\hline & & $\begin{array}{l}\text { Amount of meat at } 4-16 \mathrm{mo} \text { (total } \\
\text { grams of red [beef, pork and lamb] } \\
\text { and white [chicken, turkey, fish] meat }\end{array}$ & $\begin{array}{l}\text { MDI at } 22 \mathrm{mo} \\
(\mathrm{NSA})\end{array}$ & $\begin{array}{l}\text { PDI at } \\
22 \mathrm{mo} \\
\beta=0.73\end{array}$ & \\
\hline
\end{tabular}




\begin{tabular}{ll}
\hline consumed) & $95 \% \mathrm{Cl}:$ \\
& $0.07,1.4$, \\
$P=0.03$
\end{tabular}

1. Developmental milestone outcomes were categorized in accordance with the analytic framework, and for these studies included the following domains: cognitive, motor, and/or other development (neurological or behavioral). No studies examined social/emotional development or incidence of anxiety/depression in relation to complementary feeding (also see Figure 1).

2. The independent variables/exposures of interest were types and amounts of CFB at the age noted.

Abbreviations: BDI, behavioral development index; BF, breast-fed; BSID, Bayley Scale of Infant Development; CFB, complementary food/beverage; CFUI:

Complementary feeding utility index; DHA, docosahexaenoic acid; FF, formula-fed; IQ, intelligence quotient; MCD, milk cereal drink; MDI, mental development index; NSA, non-significant association; NSGD, no significant group differences; PDI, psychomotor development index; VEP, visual-evoked potential 


\section{Discussion}

There was insufficient evidence to draw a conclusion about the relationship between types and/or amounts of CFB consumed and developmental milestones. A grade could not be assigned due to an inadequate number of studies that were comparable in terms of design, the types of CFB that were examined, how and when developmental milestones outcomes were assessed, and the results that were reported. In addition, no conclusion could be drawn due to low generalizability, heterogeneity in exposures, observed effects, and potential confounding.

\section{Limitations}

Eight articles published since 1980 were included from three RCTs and five prospective cohort studies that examined the relationship between consuming different types and/or amounts of CFB and developmental milestones during childhood through $18 \mathrm{y}$ of age. The studies varied in terms of the types and/or amounts of CFB examined, which included dietary patterns during the complementary feeding period, meat and/or fortified-cereal intake, and foods with differing levels of DHA or phytate. Different types of outcomes were measured between 4 mo and $8.5 \mathrm{y}$ of age, which involved communication (e.g., sentence repetition, reading skills), cognitive (e.g., IQ, $\mathrm{MDI}$ ), motor (e.g., age of walking, PDI), and neurological (e.g., cortical processing) development. Two of the three RCTs noted they were slightly underpowered $(1,3)$. The direction of findings varied across the evidence. The directness with which studies examined the link between different types of CFB and developmental milestones also varied across the evidence.

Three articles $(5,6,8)$ from two observational studies identified positive associations between dietary patterns emphasizing vegetables and meats during the complementary feeding period, and IQ between ages 4-8.5 y. However, the observational nature of the studies, potential confounding, low generalizability of dietary patterns to the U.S. population, and the relatively small magnitude of the observed effects in two of the studies restrict the ability to draw a conclusion regarding the relationship between dietary patterns during the complementary feeding period and cognitive development.

Three studies (two RCTs; one observational) examined the relationship between types of CFB that may influence micronutrient absorption and developmental indices but varied in how exposures were analyzed, what types of CFB were examined, and the directness with which the studies examined the relationship between different CFB consumed and developmental milestones. Due to inconsistency in study design and results, the relationship between types and/or amounts of CFB that may influence micronutrient absorption and developmental indices remains unclear.

One high-quality study examined DHA-enriched baby food in relation to neurological development, showing positive effects of DHA-enriched baby food on visual acuity (i.e., cortical processing) (1). With only one study, however, it is not possible to draw a conclusion regarding the relationship between DHA-enriched CFB and neurological development 


\section{Research recommendations}

In order to better assess the relationship between the types and/or amounts of CFB introduced and developmental milestones, additional research may be warranted. Should research in this area be conducted, the following recommendations should be considered:

- Conduct RCTs examining how dietary patterns that are more generalizable to the U.S. population and comparable across studies are related to cognitive development

- Examine DHA-enriched CFB in relation to neurological development and other developmental milestones

- Determine whether assessment tests have predictability for longer term neurodevelopmental outcomes; it remains unclear whether achievement of developmental milestones predicts enduring effects on developmental outcomes later in childhood.

- Account for the following factors that could impact the relationship between types and/or amounts of CFB introduced and developmental milestones: the amount of human milk or formula consumed, birth weight of the infant, weight status of the infant at the time CFB and outcome assessment, age of children when assessed, and the developmental readiness of the infant.

- Explore mechanisms that could account for the relationship between types or amounts of CFB introduced and developmental outcomes

\section{Included articles}

1. Hoffman, D R, Theuer, R C, Castaneda, Y S, et al. Maturation of visual acuity is accelerated in breast-fed term infants fed baby food containing DHA-enriched egg yolk. The Journal of nutrition. 2004;134(9):2307-13.

2. Krebs, N F, Westcott, J E, Butler, N, et al. Meat as a first complementary food for breastfed infants: feasibility and impact on zinc intake and status. Journal of pediatric gastroenterology and nutrition. 2006;42(2):207-14.

3. Lind T, Persson L, Lonnerdal B, Stenlund H, Hernell O. Effects of weaning cereals with different phytate content on growth, development and morbidity: a randomized intervention trial in infants from 6 to 12 months of age. Acta paediatrica (Oslo, Norway : 1992). 2004;93(12):1575-82.

4. Dagnelie, P C, van S, W A. Macrobiotic nutrition and child health: results of a population-based, mixed-longitudinal cohort study in The Netherlands. The American journal of clinical nutrition. 1994;59(5 Suppl):1187s-96s.

5. Gale, C R, Martyn, C N, Marriott, L D, et al. Dietary patterns in infancy and cognitive and neuropsychological function in childhood. Journal of child psychology and psychiatry, and allied disciplines. 2009;50(7):816-23.

6. Golley, R K, Smithers, L G, Mittinty, M N, et al. Diet quality of U.K. infants is associated with dietary, adiposity, cardiovascular, and cognitive outcomes measured at 7-8 years of age. The Journal of nutrition. 2013;143(10):1611-7.

7. Morgan, J, Taylor, A, Fewtrell, M. Meat consumption is positively associated with psychomotor outcome in children up to 24 months of age. Journal of pediatric gastroenterology and nutrition. 2004;39(5):493-8. 
8. Smithers LG, Golley RK, Mittinty MN, Brazionis L, Northstone K, Emmett P, et al. Dietary patterns at 6,15 and 24 months of age are associated with IQ at 8 years of age. European journal of epidemiology. 2012;27(7):525-35.

\section{Other references}

9. United Nations Development Programme. Human Development Report 2014. Sustaining Human Progress: Reducing Vulnerabilities and Building Resilience. New York; 2014.

10. Centers for Disease Control and Prevention. Developmental Milestones [16 October 2017:[Available from: https://www.cdc.gov/ncbddd/actearly/milestones/index.html.

11. Briefel RR, Reidy K, Karwe V, Jankowski L, Hendricks K. Toddlers' transition to table foods: Impact on nutrient intakes and food patterns. Journal of the American Dietetic Association. 2004;104(1 Suppl 1):s38-44.

12. World Bank. The Little Data Book 2016 Washington, DC2016 [Available from: https://openknowledge.worldbank.org/handle/10986/23968. 


\section{ANALYTIC FRAMEWORK}

The analytic framework illustrates the overall scope of the review, including the population, the interventions and/or exposures, comparators, and outcomes of interest. It also includes definitions of key terms and identifies key confounders considered in the systematic review. This is the analytic framework for the systematic reviews conducted to examine the relationship between types and amounts of complementary foods and beverages consumed and developmental milestones.

Figure 1: Analytic framework

Analytic framework

- What is the relationship between types and amounts of complementary foods and beverages consumed and developmental milestones?

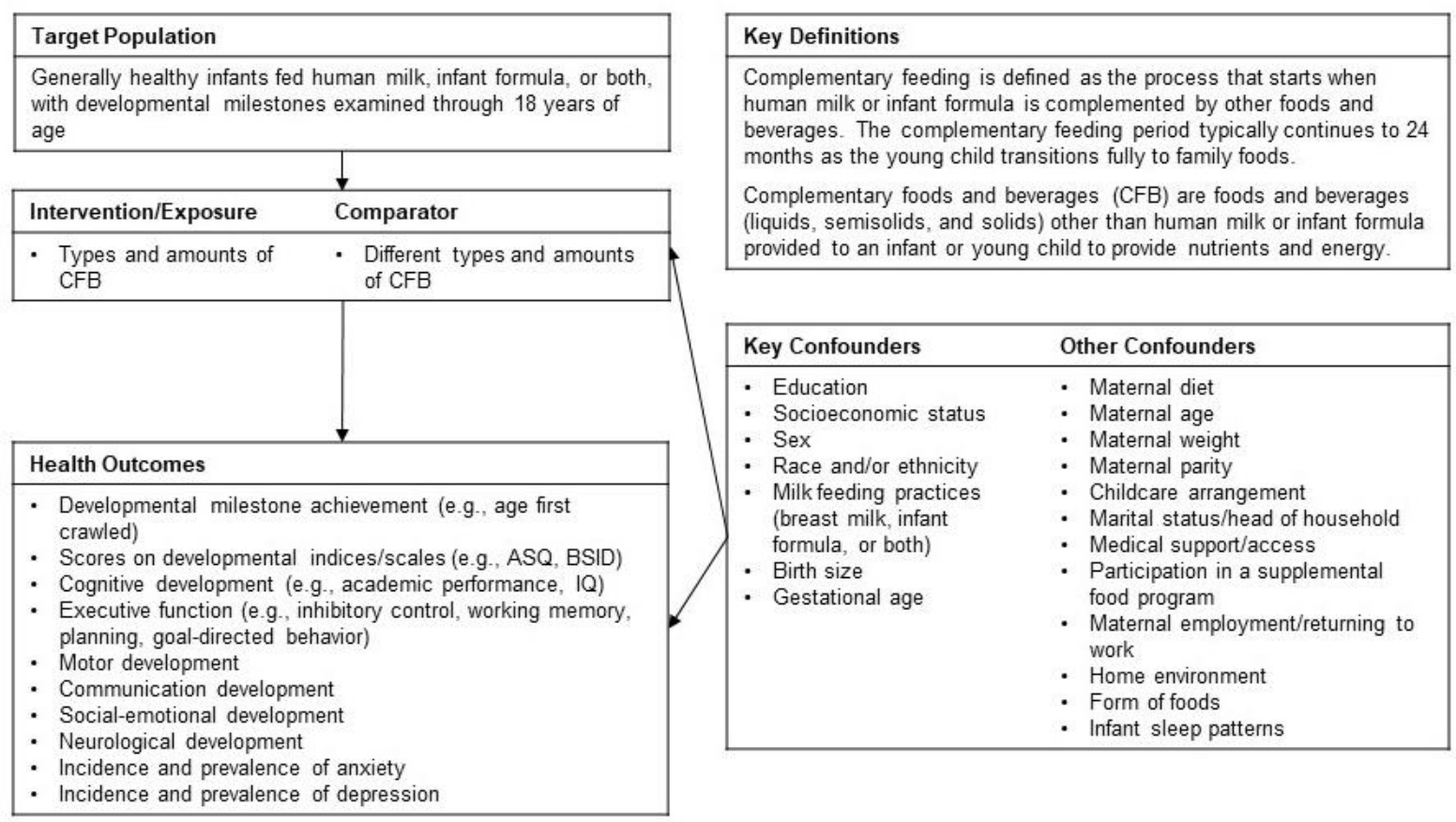

\section{SEARCH PLAN AND RESULTS}

\section{Inclusion and exclusion criteria}

This table provides the inclusion and exclusion criteria for the systematic review question(s) on the types and amounts of CFB consumed and developmental milestones. The inclusion and exclusion criteria are a set of characteristics to determine which studies will be included or excluded in the systematic review. 
Table 3. Inclusion and exclusion criteria

\begin{tabular}{|c|c|c|}
\hline Category & Inclusion Criteria & Exclusion Criteria \\
\hline Study design & $\begin{array}{ll}\text { - } & \text { RCTs } \\
\text { - } & \text { Non-RCTs } \\
\text { - } & \text { Prospective cohort studies } \\
\text { - } & \text { Retrospective cohort studies } \\
\text { - } & \text { Case-control studies } \\
\text { - } & \text { Pre/post studies with a control }\end{array}$ & $\begin{array}{ll}\text { - } & \text { Cross-sectional studies } \\
\text { - } & \text { Uncontrolled studies } \\
\text { - } & \text { Pre/post studies without a control } \\
\text { - } & \text { Narrative reviews } \\
\text { - } & \text { Systematic reviews } \\
\text { - } & \text { Meta-analyses }\end{array}$ \\
\hline $\begin{array}{l}\text { Independent } \\
\text { variable } \\
\text { (intervention or } \\
\text { exposure) }\end{array}$ & $\begin{array}{l}\text { 1. Timing of introduction to complementary foods and } \\
\text { beverages (i.e., foods and beverages other than human } \\
\text { milk or infant formula (liquids, semisolids, and solids) } \\
\text { provided to an infant or young child to provide nutrients } \\
\text { and energy) } \\
\text { 2. Types or amounts of complementary foods and } \\
\text { beverages }\end{array}$ & $\begin{array}{l}\text { Isolated consumption of: human milk; infant formulas (e.g., milk- } \\
\text { based, soy, partially-hydrolyzed, extensive-hydrolyzed, amino } \\
\text { acid-based); fluid cow's milk before } 12 \text { months of age; vitamin } \\
\text { and mineral supplements (e.g., iron drops) }\end{array}$ \\
\hline Comparator & $\begin{array}{l}\text { 1. Different timing of introduction of CFB } \\
\text { 2. Different types and amounts of CFB }\end{array}$ & $\mathrm{N} / \mathrm{A}$ \\
\hline $\begin{array}{l}\text { Dependent } \\
\text { variables } \\
\text { (outcomes) }\end{array}$ & $\begin{array}{l}\text { - } \quad \text { Developmental milestone achievement (e.g., age first } \\
\text { crawled) } \\
\text { - Scores on developmental indices/scales (e.g., ASQ, } \\
\text { BSID) } \\
\text { - Cognitive development (e.g., academic performance, } \\
\text { IQ) } \\
\text { - Executive function (e.g., inhibitory control, working } \\
\text { - } \quad \text { Social-emotional development } \\
\text { - Neurological development } \\
\text { - } \quad \text { Motor development } \\
\text { - Communication development } \\
\text { - Incidence and prevalence of anxiety }\end{array}$ & $\mathrm{N} / \mathrm{A}$ \\
\hline
\end{tabular}




\begin{tabular}{|c|c|c|}
\hline & - Incidence and prevalence of depression & \\
\hline Date range & - January 1980 - July 2016 & \\
\hline Language & - $\quad$ Studies published in English & - Studies published in languages other than English \\
\hline Publication status & - $\quad$ Studies published in peer-reviewed journals & $\begin{array}{l}\text { - Grey literature, including unpublished data, manuscripts, } \\
\text { reports, abstracts, conference proceedings }\end{array}$ \\
\hline Country $^{1}$ & $\begin{array}{l}\text { - Studies conducted in Very High or High Human } \\
\text { Development Countries }\end{array}$ & $\begin{array}{l}\text { - Studies conducted in Medium or Low Human Development } \\
\text { Countries }\end{array}$ \\
\hline Study participants & $\begin{array}{l}\text { - } \text { Human subjects } \\
\text { - } \text { Males } \\
\text { - } \text { Females }\end{array}$ & $\begin{array}{l}\text { - Hospitalized patients, not including birth and immediate } \\
\text { post-partum hospitalization of healthy babies }\end{array}$ \\
\hline $\begin{array}{l}\text { Age of study } \\
\text { participants }\end{array}$ & $\begin{array}{l}\text { - Age at intervention or exposure: } \\
\circ \text { Infants (0-12 mo) } \\
\circ \text { Toddlers (12-24 mo) } \\
\text { - Age at outcome: } \\
\circ \quad \text { Infants (0-12 mo) } \\
\circ \text { Toddlers (12-24 mo) } \\
\circ \text { Child (2-5 y) } \\
\circ \text { Child (6-12 y) } \\
\circ \text { Adolescents (13-18 y) }\end{array}$ & $\begin{array}{l}\text { - Age at intervention or exposure: } \\
\circ \text { Child (2-5 y) } \\
\circ \text { Child (6-12 y) } \\
\circ \text { Adolescents (13-18 y) } \\
\circ \text { Adults (19 and older) } \\
\circ \text { Older adults (65 to } 79 \mathrm{y}) \\
\circ \text { Older adults ( } 80+\mathrm{y}) \\
\text { Age at outcome: } \\
\circ \text { Adults (19 and older) } \\
\circ \text { Older adults (65 to } 79 \mathrm{y}) \\
\circ \text { Older adults ( } 80+\mathrm{y})\end{array}$ \\
\hline $\begin{array}{l}\text { Health status of } \\
\text { study participants }\end{array}$ & $\begin{array}{l}\text { - } \quad \text { Studies done in generally healthy populations } \\
\text { - } \quad \text { Studies done in populations where infants were full } \\
\text { term ( } \geq 37 \text { weeks gestational age) } \\
\text { - } \quad \text { Studies done in populations with elevated chronic } \\
\text { disease risk, or that enroll some participants with a }\end{array}$ & $\begin{array}{l}\text { - Studies that exclusively enroll subjects with a disease or } \\
\text { with the health outcome of interest } \\
\text { - Studies done in hospitalized or malnourished subjects }\end{array}$ \\
\hline
\end{tabular}




\begin{tabular}{l|l|l}
\hline disease or with the health outcome of interest & $\begin{array}{l}\bullet \begin{array}{l}\text { Studies of exclusively pre-term babies (gestational age }<37 \\
\text { weeks) or babies that are small for gestational age }(<2500 \mathrm{~g})\end{array} \\
\begin{array}{l}\text { Studies of subjects with infectious diseases (e.g. HIV/AIDS) } \\
\text { (or with mothers diagnosed with an infectious disease) }\end{array}\end{array}$ \\
\hline
\end{tabular}

1 The ratings of country development (e.g., high, medium, low, very low) were based on the Human Development Report 2014 (9). When a country was not included in the Human Development Report 2014 ranking, country classification from the World Bank was used instead (12). Medium Development countries were originally included, but due to concerns about generalizability to the U.S. of study participants (i.e., baseline health status) and complementary foods and beverages typically consumed, a decision was made to exclude "Medium" countries in October 2017 


\section{Search terms and electronic databases used}

\section{PubMed:}

Date(s) Searched: 7/19/2016

Search Terms:

Final:

Complementary OR supplementary OR wean* OR transition* OR introduc* OR "Infant Nutritional Physiological Phenomena"[Mesh:noexp] OR weaning[mesh] OR ((bottle*) NOT (milk OR formula))

AND (feeding* OR food OR beverage*[tiab] OR beverages[mh] OR eating OR diet[tiab] OR diet[mh] OR meal*[tiab] OR meals[mh] OR "Food and Beverages"[Mesh] OR diets[tiab] OR cereal*[tiab] OR "Edible Grain"[Mesh] OR bread*[tiab] OR whole grain* OR juice*[tiab] OR milk[tiab] OR "Milk"[Mesh] OR dairy[tiab] OR "Dairy Products"[Mesh] OR meat[tiab] OR cheese[tiab] OR yogurt[tiab] OR yoghurt* ${ }^{\star}[$ tiab] OR fruit*[tiab] OR "Fruit"[Mesh] OR vegetable*[tiab] OR "Vegetables"[Mesh] OR egg*[tiab] OR "Eggs"[Mesh] OR nut[tiab] OR nuts[tiab] OR peas[tiab] OR beans[tiab] OR legume*[tiab] OR snack*[tiab] OR bread[mh] OR honey[mh] OR vegetable*[tiab] OR "Vegetables"[Mesh] OR egg"[tiab] OR "Eggs"[Mesh:noexp] OR "egg white"[mh] OR "egg yolk"[mh] OR snack*[tiab] OR candy[mh] OR "Fast Foods"[Mesh] OR meat[mh] OR molasses[mh] OR nuts[mh] OR "Raw Foods"[Mesh] OR seeds[mh])

OR "infant food"[mesh]

OR infant feed*

OR

(breast feeding[mh] OR breastfeeding[tiab] OR breast feeding*[tiab] OR breastfeeding*[tiab] OR breastfed[tiab] OR breast-fed[tiab] OR breast-feed OR breast-feeds)

OR

(Bottle feeding[mh] OR bottle feeding ${ }^{*}[$ tiab]s OR bottle feeding OR bottle-feeding*[tiab] OR bottle-feedings OR bottle-fed[tiab] OR "bottle fed"[tiab])

NOT (editorial[ptyp] OR comment[ptyp] OR news[ptyp] OR letter[ptyp] OR review[ptyp] OR systematic[sb])

OR ((Solid food $\left.{ }^{*}\right)$ OR solids));

AND

Growth[mh:noexp] OR "Child Development"[Mesh] OR "Growth Charts"[Mesh] OR "growth and development" [Subheading] OR "growth and development"[tiab] OR "Growth and Development"[Mesh:noexp] OR "Growth"[tiab] OR development*[tiab] OR "Child Development"[Mesh] OR child develop*[tiab] OR tooth[mh] OR tooth[tiab] OR teeth[tiab] OR movement[mh] OR "Overnutrition"[Mesh] OR under-nutrition[tiab] OR undernutrition[tiab] OR "developmental delay"[tiab] OR "Motor Skills"[Mesh] OR 
"Nonverbal Communication"[Mesh]

Standing[tiab] OR sitting[tiab] OR walking[tiab] OR crawling[tiab] OR "Motor Skills"[Mesh] OR Ages and Stages Questionnaire* OR ASQ[tiab]

OR Cognitive[tiab] OR cognition[mh] OR cognition OR learning OR "Learning Disorders"[Mesh] OR "Intellectual Disability"[Mesh] OR intelligence[tiab] OR intelligence[mh] OR "Achievement"[Mesh] OR "Aptitude"[Mesh] OR "Executive Function"[Mesh] OR memory OR inhibitory control*[tiab] OR "problem solving"[tiab] OR"Social-emotional development"[tiab] OR "Neurological development"[tiab] OR "mental development"[tiab] OR "Motor development"[tiab] OR anxiety[tiab] OR anxiety[mh:noexp] OR "Anxiety, Separation"[Mesh] OR depression[tiab] OR depression[mh] OR "Depression, Postpartum"[Mesh] OR "Depressive Disorder"[Mesh] OR

"Visual Acuity"[Mesh] OR "Auditory Perception"[Mesh] OR "Psychological Tests"[Mesh]

OR

("Bone Density"[Mesh] OR "bone density"[tiab] OR "Bone Development"[Mesh] OR "Bone Development"[tiab] OR "Fractures, Bone"[Mesh] OR "Bone Diseases"[Mesh] OR osteoporosis[tiab] OR (bone[tiab] AND fracture*[tiab]) OR "Rickets"[Mesh] OR ricket $^{*}[$ tiab] OR bone mineral*[tiab] OR "bone mass"[tiab] OR bone health*[tiab] OR "Bone Demineralization, Pathologic"[Mesh] OR bone demineral*[tiab])

OR

("body size"[tiab] OR body size[mh] OR obesity[tiab] OR obese[tiab] OR overweight[mh] OR obesity[mh] OR overweight [tiab] OR adipos*[tiab] OR adiposity[mh] OR "body composition"[mh] OR body fat distribution[mh] OR "body fat"[tiab] OR "body weight"[tiab] OR body weight[mh] OR birth weight*[tiab] OR weight gain[mh] OR weight loss[mh] OR "body-weight"[tiab] OR "weight gain"[tiab] OR weightgain[tiab] OR weight loss[tiab] OR weight-loss[tiab] OR "Body Weights and Measures"[mh] OR weight[ti] OR "Anthropometry"[Mesh:noexp] OR body mass index[mh] OR "body mass index"[tiab] OR BMI[tiab] OR "weight status"[tiab] OR "adipose tissue"[mh] OR "healthy weight"[tiab] OR waist circumference[mh] OR "body mass"[ti] OR "fat mass"[tiab] OR body weight changes[mh] OR "waist circumference"[tiab] OR ideal body weight[mh] OR waist-hip ratio[mh] OR Waist Hip*[tiab] OR body height*[tiab] OR Crown-Rump Length*[tiab] OR head circumference* ${ }^{*}$ tiab] OR arm circumference ${ }^{*}[$ tiab] OR thigh circumference* OR limb circumference* OR fat free mass*[tiab] OR skinfold[tiab] OR skin fold*[tiab])

AND

infant* OR baby OR babies OR toddler* OR newborn*[tiab] OR "Child, Preschool"[Mesh] OR preschool*[tiab] OR pre-school*ttiab] OR "early childhood"[tiab] OR "early years"[tiab] OR pre-k[tiab] OR pre-primary[tiab] OR under five*[ti] OR young child*[ti] OR "head start"[tiab] OR prekindergarten[tiab] OR pre-kindergarten[tiab] OR weanling* OR limit to child, preschool 
for child 0-18 all develop outcomes

for all; body wgt/comp/bone

NOT

nutritional status[mh] OR nutritional status*[tiab] OR Nutrition Status*[tiab] OR Iron[mh] OR iron[tiab] OR "Anemia"[Mesh] OR "Anemia"[tiab] OR iron deficien*[tiab] OR ferritin*[tiab] OR ferrous[tiab] OR "Transferrin"[Mesh] OR "Transferrin"[tiab] OR zinc OR "Vitamin D"[Mesh] OR "Vitamin D"[tiab] OR "Vitamin D Deficiency"[Mesh] OR "Vitamin B 12"[Mesh] OR "Vitamin B 12"[tiab] OR "Vitamin B12"[tiab] OR "Vitamin B 12 Deficiency"[Mesh] OR Cobamide*[tiab] OR Cobalamin*[tiab] OR Cyanocobalamin[tiab] OR Folate[tiab] OR "Folic Acid"[Mesh] OR folacin[tiab] OR vitamin b9*[tiab] OR Fatty acid*[tiab] OR "Fatty Acids"[Mesh:noexp] OR fatty acid*[tiab] OR "Fatty Acids, Unsaturated"[Mesh:noexp] OR Arachidonic acid*[tiab] OR linolenic acid*[tiab] OR linoleic acid*[tiab] OR Docosahexaenoic Acid*[tiab] OR Eicosapentaenoic Acid*[tiab] OR gamma-Linolenic Acid*[tiab] OR "Arachidonic Acids"[Mesh] OR "Fatty Acids, Essential"[Mesh] OR "Fatty Acids, Omega-3"[Mesh] OR "Fatty Acids, Omega-6"[Mesh] OR alpha-Linolenic Acid*[tiab] OR "Fatty Acids, Essential"[Mesh] OR "Linolenic Acids"[Mesh] OR "Trans Fatty Acids"[Mesh] OR "Fatty Acids, Monounsaturated"[Mesh]

for nonmedline[sb]: NOT animals by: NOT (sheep[ti] OR lamb[ti] OR lambs[ti] OR calving[ti] OR calves[ti] OR mice[ti] OR mouse[ti] OR pigs[ti] OR cows[ti] OR piglets[ti] OR cow[ti] OR piglet[ti] OR monkey[ti] OR rats[ti] OR rat[ti] OR animal*[ti])

\section{Embase:}

Date(s) Searched: 8/1/16

Search Terms:

(Complementary OR supplementa* OR wean* OR transition* OR introduc* OR family) NEAR/3 (feed ${ }^{*}$ OR food ${ }^{*}$ OR beverage* OR eating OR diet)

OR

(Complementary OR transition* OR introduct* OR wean*) AND (food/exp OR 'baby food'/exp OR 'cereal'/exp OR 'dairy product'/exp OR 'egg'/exp OR 'fruit'/exp OR 'meat'/exp OR 'sea food'/exp OR 'milk'/exp OR fish/exp OR 'poultry'/exp OR 'beverage'/exp OR 'vegetable'/exp OR nut/exp OR pea/exp OR meal/exp)

OR

(Complementary OR supplementa* OR wean* OR transition* OR introduc*) NEAR/5 ('whole grain' OR 'whole grains' OR dairy OR egg OR eggs OR meat OR poultry OR seafood OR fruit* OR milk OR fish* OR poultry OR beverage* OR vegetables* OR pea OR peas OR nut OR nuts OR cereal OR bread* OR yog ${ }^{*}$ urt $^{*}$ OR cheese* OR juice* OR rice OR soup OR legume* OR snack* OR meal ${ }^{\star}$ ) (for Embase)

OR 'baby food'/de OR (solid NEAR/2 food*):ab,ti 
AND

(infant*:ti,ab OR infant/exp) OR (baby OR babies OR toddler* OR newborn* OR nurser*):ti,ab OR 'newborn'/exp OR 'newborn care'/exp OR preschool*:ti,ab OR preschool:ti,ab OR 'preschool child'/exp OR 'infancy'/exp OR "early childhood":ti,ab OR "early years" OR pre-k:ti,ab OR 'nursery'/exp OR 'nursery school'/exp OR prekindergarten:ti,ab OR pre-kindergarten:ti,ab OR weanling*

AND ([in process]/lim OR [article]/lim OR [article in press]/lim) AND ([embase]/lim NOT [medline]/lim)

AND

Limit to humans:

AND

'executive function'/exp OR 'executive function':ti,ab OR 'learning'/exp OR 'intelligence'/exp OR 'mental development'/exp OR 'mental development':ti,ab OR intelligence:ti,ab OR cogniti*:ti,ab OR 'cognition'/exp OR 'cognition assessment'/exp OR aptitude:ti,ab OR 'memory'/exp OR memory:ti,ab OR 'anxiety'/exp OR 'anxiety':ti,ab OR 'depression'/exp OR depressi*:ti,ab OR 'visual acuity'/exp OR visual:ti OR 'hearing'/exp OR hearing:ti,ab OR auditory:ti,ab OR 'postnatal development'/exp OR 'postnatal development':ti,ab OR 'overnutrition'/exp OR 'overnutrition':ti,ab OR undernutrition:ti,ab OR "developmental delay":ti,ab OR 'nonverbal communication'/exp

OR

('metabolic bone disease'/exp OR osteoporosis:ti,ab OR (bone NEAR/2 (disease* OR fracture* OR injur* OR health* OR density OR mineralize* OR demineraliz*)):ti,ab OR

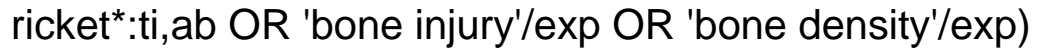

AND

'body size'/de OR 'body size':ti,ab OR 'obesity'/exp OR overweight:ab,ti OR 'macrosomia'/exp OR obese:ab,ti OR obesity:ab,ti OR 'weight gain':ab,ti OR adiposity:ab,ti OR adipose:ab,ti OR 'body weight'/exp OR 'body weight':ti,ab OR 'weight gain'/de OR 'body composition'/exp OR 'body composition':ti,ab OR 'body fat':ab,ti OR 'anthropometry'/de OR 'body mass'/de OR bmi:ab,ti OR 'body mass':ab,ti OR weight:ab,ti OR (waist NEXT/1 hip NEXT/1 ratio*) OR 'body fat'/de OR 'adipose tissue'/exp OR skinfold OR 'skin fold':ti,ab OR 'fat mass':ti,ab OR 'fat mass'/exp OR 'anthropometric parameters'/exp OR circumference OR length OR height

OR

'body growth'/exp 'body growth':ti,ab OR 'growth rate and growth regulation'/exp OR 'postnatal growth'/exp OR 'human development'/exp OR 'Bayley Scales of Infant Development'/exp OR 'cognition assessment'/exp OR 'mental function assessment'/de

\section{Cochrane:}


Date(s) Searched: 8/9/16

Search Terms:

(feed ${ }^{*}$ OR food ${ }^{*}$ OR beverage* OR diet* OR 'whole grain' OR 'whole grains' OR dairy OR egg OR meat OR poultry OR seafood OR fruit* OR milk OR fish* OR poultry OR vegetables* OR pea OR beans OR legume* OR nut OR cereal OR beverage* OR bread $^{\star}$ OR seafood OR yog ${ }^{*}$ urt $^{*}$ OR cheese OR juice OR snack OR yogurt OR yoghurt OR nut OR nuts OR honey OR meal OR meals) NEAR/3 (Complementary OR supplementa* OR wean* OR transition* OR introduct* OR family)

OR

[mh ^"Infant Nutritional Physiological Phenomena"] OR [mh weaning] OR ((bottle*) NOT (milk OR formula))

AND ([mh beverages] OR [mh eating] OR [mh diet] OR [mh meals] OR [mh "Food and Beverages"] OR [mh "Edible Grain"] OR [mh "Milk"] OR dairy[:ti,ab OR [mh "Dairy Products"] OR [mh "Fruit"] OR [mh "Vegetables"] OR [mh "Eggs"] OR [mh bread] OR [mh honey] OR [mh "Vegetables"] OR [mh ^"Eggs"] OR [mh "egg white"] OR [mh "egg yolk"] OR [mh candy] OR [mh "Fast Foods"] OR [mh meat] OR [mh molasses] OR [mh nuts] OR [mh "Raw Foods"] OR [mh seeds])

OR

((Infant* OR baby* OR babies) NEAR/2 food $\left.{ }^{\star}\right): t i$, ab OR [mh "infant food"]

AND

[mh ^Growth] OR [mh "Child Development"] OR [mh "Growth Charts"] OR "growth and development" OR [mh ^"Growth and Development"] OR [mh "Child Development"] OR (child NEAR/1 develop*):ti, ab OR [mh tooth] OR tooth:ti,ab OR teeth:ti,ab OR [mh movement] OR [mh "Overnutrition"] OR "under-nutrition:ti,ab OR undernutrition:ti,ab OR [mh "Motor Skills"] OR [mh "Nonverbal Communication"]

OR

'body growth':ti,ab OR 'growth rate and growth regulation' OR 'postnatal growth':ti,ab OR 'human development':ti,ab OR 'Bayley Scales of Infant Development'

OR Standing:ti,ab OR sitting:ti,ab OR walking:ti,ab OR crawling:ti,ab OR "Ages and Stages Questionnaire" OR ASQ:ti,ab

OR [mh cognition] OR [mh learning] OR [mh "Learning Disorders"] OR [mh "Intellectual Disability"] OR intelligence:ti,ab OR [mh intelligence] OR [mh "Achievement"] OR [mh "Aptitude"] OR [mh "Executive Function"] OR (inhibitory NEAR/1 control*):ti,ab OR "problem solving":ti,ab OR "Social-emotional development":ti,ab OR "Neurological development":ti,ab OR “mental development”:ti, ab OR "Motor development"ti,ab OR [mh ^anxiety] OR [mh "Anxiety, Separation"] OR [mh depression] OR [mh "Depression, Postpartum"] OR [mh "Depressive Disorder"] OR [mh "Visual Acuity"] OR [mh "Auditory Perception"] OR [mh "Psychological Tests"] 
OR

cogniti*:ti,ab OR aptitude:ti,ab OR memory:ti,ab OR [mh memory] OR 'anxiety':ti,ab OR depressi*:ti,ab OR visual:ti,ab OR vision:ti,ab OR hearing:ti,ab OR auditory:ti,ab OR 'postnatal development':ti,ab OR 'overnutrition':ti,ab OR "developmental delay":ti,ab OR 'nonverbal communication'

OR

[mh "Bone Density"] OR [mh "Bone Development"] OR [mh "Fractures, Bone"] OR [mh "Bone Diseases"] OR [mh "Rickets"] OR [mh "Bone Demineralization, Pathologic"] OR osteoporosis:ti,ab OR (bone NEAR/2 (disease* OR fracture* OR injur* OR health* OR density OR mineral ${ }^{*}$ OR demineral ${ }^{*}$ OR develop* OR mass)):ti,ab OR ricket*:ti,ab

OR 'body size':ti,ab OR overweight:ab,ti OR 'macrosomia':ti,ab OR obese:ab,ti OR obesity:ab,ti OR adipos*:ab,ti OR 'body weight':ti,ab OR 'weight gain':ti,ab OR 'body composition':ti, ab OR 'body fat':ab,ti OR 'anthropometr':ti,ab OR bmi:ab,ti OR 'body mass':ab,ti OR (waist NEXT/1 hip NEXT/1 ratio*) OR 'body fat':ti,ab OR 'adipose tissue':ti,ab OR skinfold:ti,ab OR 'skin fold':ti,ab OR 'fat mass':ti,ab OR circumference:ti,ab OR length:ti,ab OR height:ti,ab

([mh "body size"] OR [mh overweight] OR [mh obesity] OR [mh adiposity] OR [mh "body composition"] OR [mh "body fat distribution"] OR [mh "body weight"] OR [mh "weight gain"] OR [mh "weight loss"] OR "weight gain":ti,ab OR "weight loss":ti,ab OR "weight-loss":ti,ab OR [mh "Body Weights and Measures"] OR weight:ti OR [mh ^"Anthropometry"] OR [mh "body mass index"] OR "weight status":ti,ab OR [mh "adipose tissue"] OR "healthy weight":ti,ab OR [mh "waist circumference"] OR [mh "body weight changes"] OR [mh "ideal body weight"] OR [mh "waist-hip ratio"] OR "Waist Hip":ti,ab OR "waist-hip":ti,ab OR “Crown-Rump":ti,ab OR "fat free mass":ti,ab)

NOT (pubmed OR embase)

\section{CINAHL}

Date(s) Searched: 8/22/2016

Search Terms:

(MH "Food and Beverages+") OR (MH "Food") OR (MH "Diet") OR (MH "Eating") OR (MH "Eating Behavior") OR (MH "Taste") OR (MH "Taste Buds") OR (MH "Cereals") OR (MH "Dairy Products") OR (MH "Yogurt") OR (MH "Cheese") OR (MH "Milk") OR (MH "Eggs") OR (MH "Fruit") OR (MH "Fruit Juices") OR (MH "Meat") OR (MH "Seafood") OR (MH "Fish") OR (MH "Poultry") OR (MH "Vegetables") OR (MH "Nuts") OR (MH "Legumes") OR (MH "Bread") AND (Complementary OR supplementa* OR wean* OR transition* OR introduc ${ }^{*}$ )

OR 
('whole grain' OR 'whole grains' OR dairy OR egg OR eggs OR meat OR poultry OR seafood OR fruit* OR milk OR fish* OR poultry OR vegetables* OR pea OR peas OR nut OR nuts OR cereal OR beverage* OR bread* OR seafood OR yog* urt $^{\star}$ OR cheese $^{*}$ OR juice*) N5 (Complementary OR supplementa* OR wean* OR transition* OR introduc* OR family)

OR (Infant* OR baby OR babies) N2 food*

\section{NOT}

(MH "Nutritional Status") OR "nutritional status" OR (MH "Nutritional Requirements") OR (MH "Vitamin D") OR (MH "Vitamin D Deficiency") OR (MH "Vitamin B12 Deficiency") OR (MH "Anemia") OR "anemia" OR (MH "Anemia, Iron Deficiency") OR (MH "Iron") OR (MH "Zinc") OR (MH "Vitamin B12") OR (MH "Vitamin B12 Deficiency") OR (MH "Folic Acid") OR (MH "Niacin") OR (MH "Folic Acid Deficiency") OR "folate" OR "folacin" OR cyanocobalamin* OR cobalamin* OR cobamamide* OR (MH "Fatty Acids") OR "fatty acids" OR (MH "Fatty Acids, Omega-6") OR (MH "Fatty Acids, Omega-3") OR (MH "Fatty Acids, Unsaturated") OR (MH "Trans Fatty Acids") OR (MH "Fatty Acids, Monounsaturated") OR (MH "Fatty Acids, Saturated") OR (MH "Fatty Acids, Essential") OR (MH "Arachidonic Acids") OR (MH "Docosahexaenoic Acids") OR (MH "Linolenic Acids") OR (MH "Linoleic Acids")

AND

osteoporosis OR (bone n2 (disease* OR fracture* OR injur* OR health* OR density OR mineralize* OR demineraliz*)) OR ricket* OR (MH "Osteoporosis") OR (MH "Bone Density") OR

(MH "Bone Diseases+") OR (MH "Bone Diseases, Developmental+") OR (MH "Rickets+")

OR

(MH "Executive Function") OR (MH "Learning+") OR (MH "Intelligence+") OR "intelligence" OR (MH "Intelligence Tests") OR (MH "Cognition+") OR "cognition" OR "mental development" OR (MH "Aptitude") OR "aptitude" OR (MH "Aptitude Tests+") OR (MH "Memory+") OR "memory" OR (MH "Anxiety+") OR "anxiety" OR (MH "Depression+") OR "depression" OR (MH "Visual Acuity") OR (MH "Visual Perception+") OR (MH "Hearing+") OR "auditory" OR "overnutrition" OR "undernutrition" OR (MH "Nonverbal Communication+") OR "postnatal development" OR OR "developmental delay"

OR

(MH "Anthropometry+") OR (MH "Body Weights and Measures+") OR (MH "Body Weight+") OR

(MH "Bone Development+") OR (MH "Growth+") OR (MH "Human Development+") OR "bayley scales" OR "mental function" OR (MH "Body Size") OR (MH "Obesity+") OR "overweight" OR "macrosomia" OR (MH "Weight Gain+") OR (MH "Waist-Hip Ratio") OR (MH "Body Composition+") OR (MH "Adipose Tissue+") OR (MH "Abdominal Fat") OR (MH "Fat Free Mass") OR (MH "Body Mass Index") OR (MH "Skinfold Thickness") 
OR (MH "Head Circumference") OR (MH "Arm Circumference") OR (MH "Waist Circumference") OR (MH "Growth and Development (Omaha)") OR (MH "Body Height") OR (MH "Crown-Rump Length") OR (MH "Leg Length Inequality") OR (MH "Mean Length of Utterance")

Figure 2: Flow chart of literature search and screening results

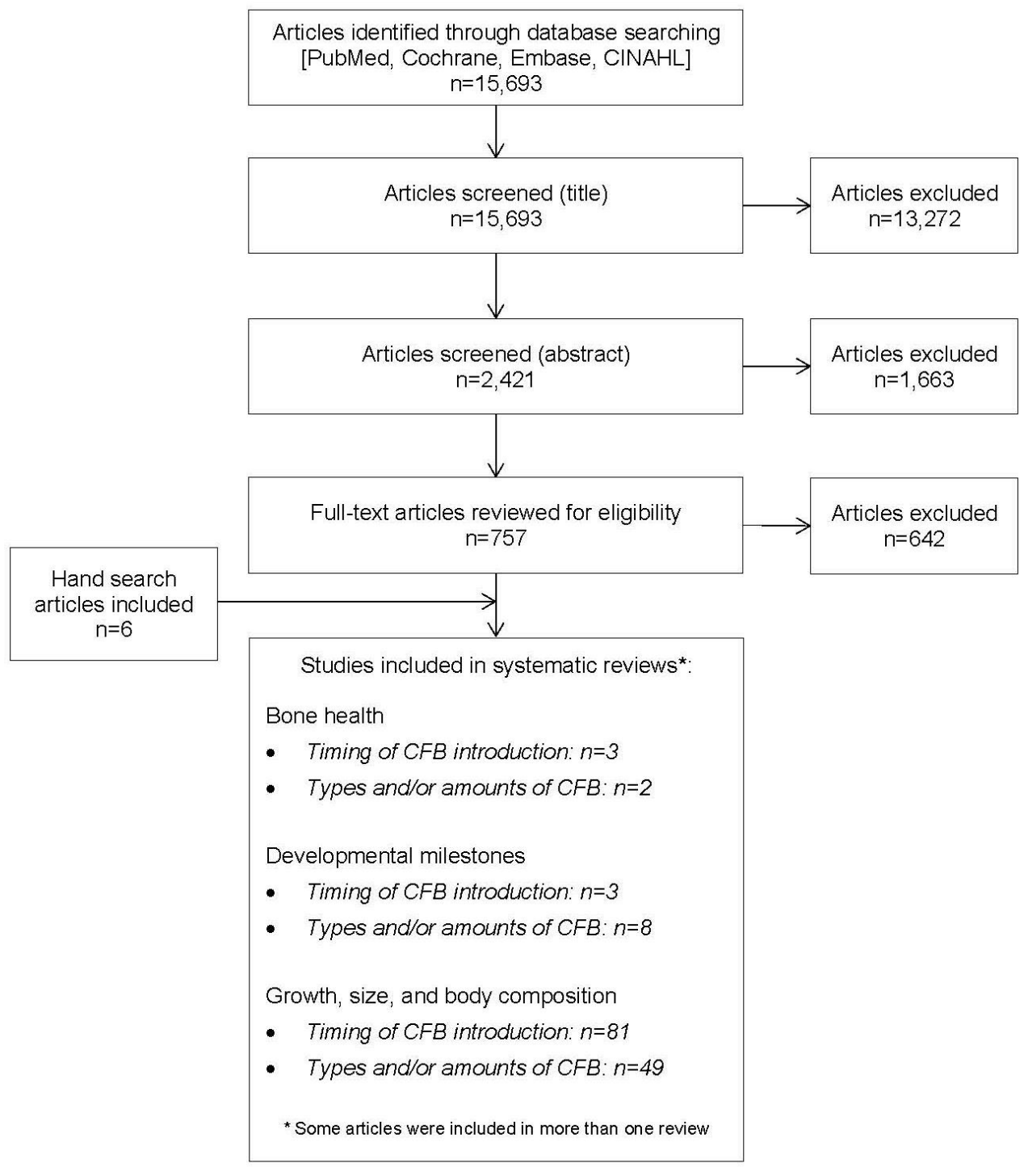

This flow chart illustrates the literature search results for articles examining the relationship between complementary feeding and developmental milestones. The results of an electronic database search were screened independently by two NESR analysts by reviewing titles, abstracts, and full text articles to determine which articles met the criteria for inclusion. A manual search was done to ascertain articles not identified through the electronic database search. The systematic review on timing of introduction of CFB included 3 articles, and the systematic review on types and amounts of CFB consumed included 8 articles. The literature search was conducted for multiple systematic reviews that addressed complementary feeding and various health outcomes. 


\section{Excluded articles}

The table below lists the excluded articles with at least one reason for exclusion, but may not reflect all possible reasons.

Table 4. Excluded articles

\begin{tabular}{|c|c|c|}
\hline & Citation & Rationale $^{1}$ \\
\hline 1 & Complementary feeding in the WHO Multicentre Growth Reference Study. Acta Paediatr Suppl.2006;450:27-37. & DV \\
\hline 2 & $\begin{array}{l}\text { Weaning and the weaning diet. Report of the Working Group on the Weaning Diet of the Committee on Medical Aspects of Food } \\
\text { Policy. Rep Health Soc Subj (Lond). } 1994 ; 45: 1-113 \text {. }\end{array}$ & Design \\
\hline 3 & $\begin{array}{l}\text { Aarts, C.,Kylberg, E.,Hofvander, Y.,Gebre-Medhin, M. Growth under privileged conditions of healthy Swedish infants exclusively } \\
\text { breastfed from birth to } 4-6 \text { months: a longitudinal prospective study based on daily records of feeding. Acta } \\
\text { Paediatr.2003;92:145-51. }\end{array}$ & IV \\
\hline 4 & $\begin{array}{l}\text { Abarin, T.,Yan Wu, Y.,Warrington, N.,Lye, S.,Pennell, C.,Briollais, L. The impact of breastfeeding on FTO-related BMI growth } \\
\text { trajectories: an application to the Raine pregnancy cohort study. Int J Epidemiol.2012;41:1650-60. }\end{array}$ & IV \\
\hline 5 & $\begin{array}{l}\text { Abou Samra, H.,Stevens, D.,Binkley, T.,Specker, B. Determinants of bone mass and size in 7-year-old former term, late-preterm, } \\
\text { and preterm boys. Osteoporos Int.2009;20:1903-10. }\end{array}$ & Design, IV \\
\hline 6 & $\begin{array}{l}\text { Aboud, F. E.,Akhter, S. A cluster-randomized evaluation of a responsive stimulation and feeding intervention in bangladesh. } \\
\text { Pediatrics.2011;127:e1191-7. }\end{array}$ & IV \\
\hline 7 & $\begin{array}{l}\text { Aboud, F. E.,Shafique, S.,Akhter, S. A responsive feeding intervention increases children's self-feeding and maternal } \\
\text { responsiveness but not weight gain. J Nutr.2009;139:1738-43. }\end{array}$ & IV \\
\hline 8 & $\begin{array}{l}\text { Adu-Afarwuah, S.,Lartey, A.,Brown, K. H.,Zlotkin, S.,Briend, A.,Dewey, K. G. Randomized comparison of } 3 \text { types of micronutrient } \\
\text { supplements for home fortification of complementary foods in Ghana: effects on growth and motor development. Am J Clin } \\
\text { Nutr.2007;86:412-20. }\end{array}$ & IV \\
\hline 9 & $\begin{array}{l}\text { Agarwal, K. N.,Agarwal, D. K.,Gupta, A.,Bansal, A. K. Relationship of exclusive breast feeding for } 6 \text { mo to linear growth up to } 18 \\
\text { mo of age. Indian J Pediatr.2013;80:11-5. }\end{array}$ & Country \\
\hline 10 & $\begin{array}{l}\text { Aggarwal, A.,Arora, S.,Patwari, A. K. Breastfeeding among urban women of low-socioeconomic status: factors influencing } \\
\text { introduction of supplemental feeds before four months of age. Indian Pediatr.1998;35:269-73. }\end{array}$ & $\begin{array}{l}\text { Design, } \\
\text { IV,DV }\end{array}$ \\
\hline 11 & $\begin{array}{l}\text { Agostoni, C.,Fiocchi, A.,Riva, E.,Terracciano, L.,Sarratud, T.,Martelli, A.,Lodi, F.,D'Auria, E.,Zuccotti, G.,Giovannini, M. Growth of } \\
\text { infants with IgE-mediated cow's milk allergy fed different formulas in the complementary feeding period. Pediatr Allergy } \\
\text { Immunol.2007;18:599-606. }\end{array}$ & IV \\
\hline 12 & $\begin{array}{l}\text { Agostoni, C.,Grandi, F.,Gianni, M. L.,Silano, M.,Torcoletti, M.,Giovannini, M.,Riva, E. Growth patterns of breast fed and formula } \\
\text { fed infants in the first } 12 \text { months of life: an Italian study. Arch Dis Child.1999;81:395-9. }\end{array}$ & IV \\
\hline 13 & Agostoni, C.,Grandi, F.,Scaglioni, S.,Gianni, M. L.,Torcoletti, M.,Radaelli, G.,Fiocchi, A.,Riva, E. Growth pattern of breastfed and & IV \\
\hline
\end{tabular}




\begin{tabular}{|c|c|c|}
\hline & nonbreastfed infants with atopic dermatitis in the first year of life. Pediatrics.2000;106:E73. & \\
\hline 14 & $\begin{array}{l}\text { Agostoni,C.,Marangoni,F.,Lammardo,A. M.,Giovannini,M.,Riva,E.,Galli,C. Breastfeeding duration, milk fat composition and } \\
\text { developmental indices at } 1 \text { year of life among breastfed infants. Prostaglandins Leukot Essent Fatty Acids.2001;64:105-9. }\end{array}$ & IV \\
\hline 15 & $\begin{array}{l}\text { Agostoni,C.,Zuccotti,G. V.,Radaelli,G.,Besana,R.,Podesta,A.,Sterpa,A.,Rottoli,A.,Riva,E.,Giovannini,M. Docosahexaenoic acid } \\
\text { supplementation and time at achievement of gross motor milestones in healthy infants: a randomized, prospective, double-blind, } \\
\text { placebo-controlled trial. Am J Clin Nutr.2009;89:64-70. }\end{array}$ & IV \\
\hline 16 & $\begin{array}{l}\text { Allen, L.,Shrimpton, R. The International Research on Infant Supplementation study: implications for programs and further } \\
\text { research. J Nutr.2005;135:666s-669s. }\end{array}$ & Design \\
\hline 17 & $\begin{array}{l}\text { Alm,B.,Aberg,N.,Erdes,L.,Mollborg,P.,Pettersson,R.,Norvenius,S. G.,Goksor,E.,Wennergren,G. Early introduction of fish } \\
\text { decreases the risk of eczema in infants. Arch Dis Child.2009;94:11-5. }\end{array}$ & DV \\
\hline 18 & $\begin{array}{l}\text { Almqvist,C.,Garden,F.,Xuan,W.,Mihrshahi,S.,Leeder,S. R.,Oddy,W.,Webb,K.,Marks,G. B. Omega-3 and omega-6 fatty acid } \\
\text { exposure from early life does not affect atopy and asthma at age } 5 \text { years. J Allergy Clin Immunol.2007;119:1438-44. }\end{array}$ & IV, DV \\
\hline 19 & $\begin{array}{l}\text { Alvarez-Uria, G.,Midde, M.,Pakam, R.,Bachu, L.,Naik, P. K. Effect of Formula Feeding and Breastfeeding on Child Growth, Infant } \\
\text { Mortality, and HIV Transmission in Children Born to HIV-Infected Pregnant Women Who Received Triple Antiretroviral Therapy in } \\
\text { a Resource-Limited Setting: Data from an HIV Cohort Study in India. ISRN Pediatr.2012;2012:763591. }\end{array}$ & $\begin{array}{l}\text { Health } \\
\text { statu }\end{array}$ \\
\hline 20 & $\begin{array}{l}\text { Andersen, L. B.,Molgaard, C.,Michaelsen, K. F.,Carlsen, E. M.,Bro, R.,Pipper, C. B. Indicators of dietary patterns in Danish } \\
\text { infants at } 9 \text { months of age. Food Nutr Res.2015;59:27665. }\end{array}$ & Design \\
\hline 21 & $\begin{array}{l}\text { Andersen,A. D.,Michaelsen,K. F.,Hellgren,L. I.,Trolle,E.,Lauritzen,L. A randomized controlled intervention with fish oil versus } \\
\text { sunflower oil from } 9 \text { to } 18 \text { months of age: exploring changes in growth and skinfold thicknesses. Pediatr Res.2011;70:368-74. }\end{array}$ & IV \\
\hline 22 & $\begin{array}{l}\text { Andersen,L. B.,Pipper,C. B.,Trolle,E.,Bro,R.,Larnkjaer,A.,Carlsen,E. M.,Molgaard,C.,Michaelsen,K. F. Maternal obesity and } \\
\text { offspring dietary patterns at } 9 \text { months of age. Eur J Clin Nutr.2015;69:668-75. }\end{array}$ & DV \\
\hline 23 & $\begin{array}{l}\text { Anderson, G. H.,Morson-Pasut, L. A.,Bryan, H.,Cleghorn, G., Tanaka, P.,Yeung, D.,Zimmerman, B. Age of introduction of cow's } \\
\text { milk to infants. J Pediatr Gastroenterol Nutr.1985;4:692-8. }\end{array}$ & Design \\
\hline 24 & $\begin{array}{l}\text { Anderson, V. P.,Cornwall, J.,Jack, S.,Gibson, R. S. Intakes from non-breastmilk foods for stunted toddlers living in poor urban } \\
\text { villages of Phnom Penh, Cambodia, are inadequate. Matern Child Nutr.2008;4:146-59. }\end{array}$ & $\begin{array}{l}\text { Design, He } \\
\text { alth status }\end{array}$ \\
\hline 25 & $\begin{array}{l}\text { Andres, A.,Casey, P. H.,Cleves, M. A.,Badger, T. M. Body fat and bone mineral content of infants fed breast milk, cow's milk } \\
\text { formula, or soy formula during the first year of life. J Pediatr.2013;163:49-54. }\end{array}$ & IV \\
\hline 26 & $\begin{array}{l}\text { Andres, A.,Cleves, M. A.,Bellando, J. B.,Pivik, R. T.,Casey, P. H.,Badger, T. M. Developmental status of 1-year-old infants fed } \\
\text { breast milk, cow's milk formula, or soy formula. Pediatrics.2012;129:1134-40. }\end{array}$ & IV \\
\hline 27 & $\begin{array}{l}\text { Andrissi, L.,Mottini, G.,Sebastiani, V.,Boldrini, L.,Giuliani, A. Dietary habits and growth: an urban/rural comparison in the Andean } \\
\text { region of Apurimac, Peru. Ann Ist Super Sanita.2013;49:340-6. }\end{array}$ & IV \\
\hline 28 & Anfield,L. Nutrition in the first year. Midwife Health Visit Community Nurse.1985;21:161-4. & Design \\
\hline
\end{tabular}




\begin{tabular}{|c|c|c|}
\hline 29 & $\begin{array}{l}\text { Anzman-Frasca, S., Liu, S.,Gates, K. M.,Paul, I. M.,Rovine, M. J.,Birch, L. L. Infants' Transitions out of a Fussing/Crying State Are } \\
\text { Modifiable and Are Related to Weight Status. Infancy.2013;18:662-686. }\end{array}$ & IV \\
\hline 30 & Armstrong, J.,Reilly, J. J. Breastfeeding and lowering the risk of childhood obesity. Lancet.2002;359:2003-4. & IV \\
\hline 31 & $\begin{array}{l}\text { Arsenault,J. E.,Havel,P. J.,Lopez de Romana,D.,Penny,M. E.,Van Loan,M. D.,Brown,K. H. Longitudinal measures of circulating } \\
\text { leptin and ghrelin concentrations are associated with the growth of young Peruvian children but are not affected by zinc } \\
\text { supplementation. Am J Clin Nutr.2007;86:1111-9. }\end{array}$ & $\begin{array}{l}\text { Health } \\
\text { status }\end{array}$ \\
\hline 32 & Arvas, A.,Elgormus, Y.,Gur, E.,Alikasifoglu, M.,Celebi, A. Iron status in breast-fed full-term infants. Turk J Pediatr.2000;42:22-6. & IV \\
\hline 33 & $\begin{array}{l}\text { Asha Bai, P. V.,Leela, M.,Subramaniam, V. R. Adequacy of breast milk for optimal growth of infants. Trop Geogr } \\
\text { Med.1980;32:158-62. }\end{array}$ & IV \\
\hline 34 & $\begin{array}{l}\text { Assuncao, M. L.,Ferreira, H. S.,Coutinho, S. B.,Santos, L. M.,Horta, B. L. Protective effect of breastfeeding against overweight } \\
\text { can be detected as early as the second year of life: a study of children from one of the most socially-deprived areas of Brazil. J } \\
\text { Health Popul Nutr.2015;33:85-91. }\end{array}$ & $\begin{array}{l}\text { Design, } \\
\text { Health } \\
\text { status, IV }\end{array}$ \\
\hline 35 & $\begin{array}{l}\text { Atladottir, H.,Thorsdottir, I. Energy intake and growth of infants in Iceland-a population with high frequency of breast-feeding and } \\
\text { high birth weight. Eur J Clin Nutr.2000;54:695-701. }\end{array}$ & IV \\
\hline 36 & Auestad,N. Infant nutrition--brain development--disease in later life. An introduction. Dev Neurosci.2000;22:472-3. & Design \\
\hline 37 & $\begin{array}{l}\text { Augusto,R. A.,Souza,J. M. Effectiveness of a supplementary feeding program in child weight gain. Rev Saude } \\
\text { Publica.2010;44:793-801. }\end{array}$ & Design, IV \\
\hline 38 & $\begin{array}{l}\text { Axelsson,I. E.,Jakobsson,I.,Raiha,N. C. Formula with reduced protein content: effects on growth and protein metabolism during } \\
\text { weaning. Pediatr Res.1988;24:297-301. }\end{array}$ & IV \\
\hline 39 & $\begin{array}{l}\text { Azad, M. B.,Konya, T.,Maughan, H.,Guttman, D. S.,Field, C. J.,Chari, R. S.,Sears, M. R.,Becker, A. B.,Scott, J. A.,Kozyrskyj, A. } \\
\text { L. Gut microbiota of healthy Canadian infants: profiles by mode of delivery and infant diet at } 4 \text { months. Cmaj.2013;185:385-94. }\end{array}$ & DV \\
\hline 40 & Badger, T. Effects of soy infant formula on growth and development in the first year of life. Food Nutr Bull.2013;34:252-3. & Design, IV \\
\hline 41 & $\begin{array}{l}\text { Bahamondes L,Bahamondes MV,Modesto W,Tilley IB,Magalhaes A,Pinto e Silva JL,Amaral E, Jr. Mishell DR. Effect of hormonal } \\
\text { contraceptives during breastfeeding on infant's milk ingestion and growth. Fertil Steril.2013;100:445-50. }\end{array}$ & IV \\
\hline 42 & $\begin{array}{l}\text { Bai, K. I.,Sastry, V. N.,Reddy, C. C. A comparative study of feeding pattern of infants in rural and urban areas. Indian J } \\
\text { Pediatr.1981;48:277-80. }\end{array}$ & Design, IV \\
\hline 43 & $\begin{array}{l}\text { Balaban, G.,Motta, M. E.,Silva, G. A. Early weaning and other potential risk factors for overweight among preschool children. } \\
\text { Clinics (Sao Paulo).2010;65:181-7. }\end{array}$ & IV, Age \\
\hline 44 & $\begin{array}{l}\text { Balogun,T. A.,Lombard,M. J.,McLachlan,M. The nutrient intake of children aged 12-36 months living in two communities in the } \\
\text { Breede Valley, Western Cape province, South Africa. South African Family Practice.2015;57:1-7 7p. }\end{array}$ & Design \\
\hline 45 & $\begin{array}{l}\text { Baranowski, T.,Bryan, G. T.,Harrison, J. A.,Rassin, D. K.,Greaves, K. A.,Baranowski, J. H. Height, infant-feeding practices and } \\
\text { cardiovascular functioning among } 3 \text { or } 4 \text { year old children in three ethnic groups. J Clin Epidemiol.1992;45:513-8. }\end{array}$ & DV \\
\hline
\end{tabular}




\begin{tabular}{|c|c|c|}
\hline 46 & $\begin{array}{l}\text { Bartok, C. J.,Schaefer, E. W.,Beiler, J. S.,Paul, I. M. Role of body mass index and gestational weight gain in breastfeeding } \\
\text { outcomes. Breastfeed Med.2012;7:448-56. }\end{array}$ & IV, DV \\
\hline 47 & Beal, V. A. Nutrition and growth-patterns of young children. ASDC J Dent Child.1983;50:139-41. & Design \\
\hline 48 & $\begin{array}{l}\text { Begum, H. A.,Mascie-Taylor, C.,Nahar, S. The impact of food supplementation on infant weight gain in rural Bangladesh; an } \\
\text { assessment of the Bangladesh Integrated Nutritional Program (BINP). Public Health Nutr.2007;10:49-54. }\end{array}$ & IV \\
\hline 49 & $\begin{array}{l}\text { Beinner,M. A.,Velasquez-Melendez,G.,Pessoa,M. C.,Greiner,T. Iron-fortified rice is as efficacious as supplemental iron drops in } \\
\text { infants and young children. J Nutr.2010;140:49-53. }\end{array}$ & IV, DV \\
\hline 50 & $\begin{array}{l}\text { Ben,X. M.,Zhou,X. Y.,Zhao,W. H.,Yu,W. L.,Pan,W.,Zhang,W. L.,Wu,S. M.,Van Beusekom,C. M.,Schaafsma,A. Growth and } \\
\text { development of term infants fed with milk with long-chain polyunsaturated fatty acid supplementation. Chin Med J } \\
\text { (Engl).2004;117:1268-70. }\end{array}$ & IV \\
\hline 51 & $\begin{array}{l}\text { Bennett,W. E.,Jr.,Hendrix,K. S.,Thompson-Fleming,R. T.,Downs,S. M.,Carroll,A. E. Early cow's milk introduction is associated } \\
\text { with failed personal-social milestones after } 1 \text { year of age. Eur J Pediatr.2014;173:887-92. }\end{array}$ & IV \\
\hline 52 & $\begin{array}{l}\text { Bergmann, K. E.,Bergmann, R. L., Von Kries, R.,Bohm, O.,Richter, R.,Dudenhausen, J. W.,Wahn, U. Early determinants of } \\
\text { childhood overweight and adiposity in a birth cohort study: role of breast-feeding. Int J Obes Relat Metab Disord.2003;27:162-72. }\end{array}$ & IV \\
\hline 53 & $\begin{array}{l}\text { Bernal, M. J.,Periago, M. J.,Martinez, R.,Ortuno, I.,Sanchez-Solis, M.,Ros, G.,Romero, F.,Abellan, P. Effects of infant cereals with } \\
\text { different carbohydrate profiles on colonic function--randomised and double-blind clinical trial in infants aged between } 6 \text { and } 12 \\
\text { months--pilot study. Eur J Pediatr.2013;172:1535-42. }\end{array}$ & IV \\
\hline 54 & $\begin{array}{l}\text { Berni Canani R,Nocerino R,Terrin G,Frediani T,Lucarelli S,Cosenza L,Passariello A,Leone L,Granata V,Di Costanzo M,Pezzella } \\
\text { V,Troncone R. Formula selection for management of children with cow's milk allergy influences the rate of acquisition of } \\
\text { tolerance: a prospective multicenter study. J Pediatr.2013;163:771-7.e1. }\end{array}$ & IV, DV \\
\hline 55 & $\begin{array}{l}\text { Betoko, A.,Charles, M. A.,Hankard, R.,Forhan, A.,Bonet, M.,Regnault, N.,Botton, J.,Saurel-Cubizolles, M. J.,de Lauzon-Guillain, } \\
\text { B. Determinants of infant formula use and relation with growth in the first } 4 \text { months. Matern Child Nutr.2014;10:267-79. }\end{array}$ & IV \\
\hline 56 & $\begin{array}{l}\text { Betoko, A.,Charles, M. A.,Hankard, R.,Forhan, A.,Bonet, M.,Saurel-Cubizolles, M. J.,Heude, B.,de Lauzon-Guillain, B. Infant } \\
\text { feeding patterns over the first year of life: influence of family characteristics. Eur J Clin Nutr.2013;67:631-7. }\end{array}$ & DV \\
\hline 57 & $\begin{array}{l}\text { Bhandari, N.,Bahl, R.,Nayyar, B.,Khokhar, P.,Rohde, J. E.,Bhan, M. K. Food supplementation with encouragement to feed it to } \\
\text { infants from } 4 \text { to } 12 \text { months of age has a small impact on weight gain. J Nutr.2001;131:1946-51. }\end{array}$ & Country \\
\hline 58 & $\begin{array}{l}\text { Bhandari, N.,Mazumder, S.,Bahl, R.,Martines, J.,Black, R. E.,Bhan, M. K. An educational intervention to promote appropriate } \\
\text { complementary feeding practices and physical growth in infants and young children in rural Haryana, India. J } \\
\text { Nutr.2004;134:2342-8. }\end{array}$ & IV \\
\hline 59 & $\begin{array}{l}\text { Bhatia, B. D.,Banerjee, D.,Agarwal, D. K.,Agarwal, K. N. Exterogestate growth: relationship with maternal body size and dietary } \\
\text { intakes. Indian J Pediatr.1983;50:241-6. }\end{array}$ & $\begin{array}{l}\text { Health } \\
\text { status, IV }\end{array}$ \\
\hline 60 & $\begin{array}{l}\text { Bindon, J. R.,Cabrera, C. Infant feeding patterns and growth of infants in American Samoa during the first year of life. Hum } \\
\text { Biol.1988;60:81-92. }\end{array}$ & $\begin{array}{l}\text { Design, IV, } \\
\text { DV }\end{array}$ \\
\hline
\end{tabular}




\begin{tabular}{|c|c|c|}
\hline 61 & $\begin{array}{l}\text { Bindon, J. R. The body build and composition of Samoan children: relationships to infant feeding patterns and infant weight-for- } \\
\text { length status. Am J Phys Anthropol.1984;63:379-88. }\end{array}$ & IV \\
\hline 62 & Bindon, J. R. The influence of infant feeding patterns on growth of children in American Samoa. Med Anthropol.1985;9:183-95. & Country \\
\hline 63 & $\begin{array}{l}\text { Birch,E. E.,Garfield,S.,Castaneda,Y.,Hughbanks-Wheaton,D.,Uauy,R.,Hoffman,D. Visual acuity and cognitive outcomes at } 4 \\
\text { years of age in a double-blind, randomized trial of long-chain polyunsaturated fatty acid-supplemented infant formula. Early Hum } \\
\text { Dev.2007;83:279-84. }\end{array}$ & IV \\
\hline 64 & $\begin{array}{l}\text { Bisimwa, G.,Owino, V. O.,Bahwere, P.,Dramaix, M.,Donnen, P.,Dibari, F.,Collins, S. Randomized controlled trial of the } \\
\text { effectiveness of a soybean-maize-sorghum-based ready-to-use complementary food paste on infant growth in South Kivu, } \\
\text { Democratic Republic of Congo. Am J Clin Nutr.2012;95:1157-64. }\end{array}$ & IV \\
\hline 65 & $\begin{array}{l}\text { Bjorke-Monsen, A. L. Is exclusive breastfeeding ensuring an optimal micronutrient status and psychomotor development in } \\
\text { infants?. Clin Biochem.2014;47:714. }\end{array}$ & Design \\
\hline 66 & Block,S. L. Delayed introduction of solid foods to infants: not so fast!. Pediatr Ann.2013;42:143-7. & Design \\
\hline 67 & $\begin{array}{l}\text { Bogen, D. L., Hanusa, B. H.,Whitaker, R. C. The effect of breast-feeding with and without formula use on the risk of obesity at } 4 \\
\text { years of age. Obes Res.2004;12:1527-35. }\end{array}$ & IV \\
\hline 68 & $\begin{array}{l}\text { Bonuck, K.,Avraham, S. B.,Lo, Y.,Kahn, R.,Hyden, C. Bottle-weaning intervention and toddler overweight. J } \\
\text { Pediatr.2014;164:306-12.e1-2. }\end{array}$ & IV \\
\hline 69 & $\begin{array}{l}\text { Borschel, M. W.,Baggs, G. E.,Barrett-Reis, B. Growth of healthy term infants fed ready-to-feed and powdered forms of an } \\
\text { extensively hydrolyzed casein-based infant formula: a randomized, blinded, controlled trial. Clin Pediatr (Phila).2014;53:585-92. }\end{array}$ & IV \\
\hline 70 & $\begin{array}{l}\text { Boulton J. Nutrition in childhood and its relationships to early somatic growth, body fat, blood pressure, and physical fitness. Acta } \\
\text { Paediatr Scand Suppl.1981;284:1-85. }\end{array}$ & Design,DV \\
\hline 71 & $\begin{array}{l}\text { Brakohiapa, L. A.,Yartey, J.,Bille, A., Harrison, E.,Quansah, E.,Armar, M. A.,Kishi, K.,Yamamoto, S. Does prolonged } \\
\text { breastfeeding adversely affect a child's nutritional status?. Lancet.1988;2:416-8. }\end{array}$ & IV, DV \\
\hline 72 & $\begin{array}{l}\text { Brazionis, L.,Golley, R. K.,Mittinty, M. N.,Smithers, L. G.,Emmett, P.,Northstone, K., Lynch, J. W. Diet spanning infancy and } \\
\text { toddlerhood is associated with child blood pressure at age } 7.5 \text { y. Am J Clin Nutr.2013;97:1375-86. }\end{array}$ & DV \\
\hline 73 & $\begin{array}{l}\text { Breij, L. M.,Mulder, M. T.,van Vark-van der Zee, L. C.,Hokken-Koelega, A. C. Appetite-regulating hormones in early life and } \\
\text { relationships with type of feeding and body composition in healthy term infants. Eur J Nutr.2016. }\end{array}$ & IV \\
\hline 74 & $\begin{array}{l}\text { Brekke, H. K.,van Odijk, J.,Ludvigsson, J. Predictors and dietary consequences of frequent intake of high-sugar, low-nutrient } \\
\text { foods in 1-year-old children participating in the ABIS study. Br J Nutr.2007;97:176-81. }\end{array}$ & DV \\
\hline 75 & $\begin{array}{l}\text { Brew,B. K.,Toelle,B. G.,Webb,K. L.,Almqvist,C.,Marks,G. B. Omega-3 supplementation during the first } 5 \text { years of life and later } \\
\text { academic performance: a randomised controlled trial. Eur J Clin Nutr.2015;69:419-24. }\end{array}$ & IV \\
\hline 76 & $\begin{array}{l}\text { Briend,A.,Bari,A. Breastfeeding improves survival, but not nutritional status, of } 12-35 \text { months old children in rural Bangladesh. } \\
\text { Eur J Clin Nutr.1989;43:603-8. }\end{array}$ & $\begin{array}{l}\text { Health } \\
\text { status, IV }\end{array}$ \\
\hline
\end{tabular}




\begin{tabular}{|c|c|c|}
\hline 77 & $\begin{array}{l}\text { Briend,A.,Darmon,N. Determining limiting nutrients by linear programming: A new approach to predict insufficient intakes from } \\
\text { complementary foods. Pediatrics.2000;106:1288-9. }\end{array}$ & Design \\
\hline 78 & $\begin{array}{l}\text { Brito,A.,Olivares,M.,Pizarro,T.,Rodriguez,L.,Hertrampf,E. Chilean complementary feeding program reduces anemia and improves } \\
\text { iron status in children aged } 11 \text { to } 18 \text { months. Food Nutr Bull.2013;34:378-85. }\end{array}$ & Design \\
\hline 79 & Brown A,Lee MD. Early influences on child satiety-responsiveness: the role of weaning style. Pediatr Obes.2015;10:57-66. & IV \\
\hline 80 & $\begin{array}{l}\text { Brown, A.,Lee, M. Breastfeeding during the first year promotes satiety responsiveness in children aged 18-24 months. Pediatr } \\
\text { Obes.2012;7:382-90. }\end{array}$ & IV \\
\hline 81 & $\begin{array}{l}\text { Brown,K. H.,Lopez de Romana,D.,Arsenault,J. E.,Peerson,J. M.,Penny,M. E. Comparison of the effects of zinc delivered in a } \\
\text { fortified food or a liquid supplement on the growth, morbidity, and plasma zinc concentrations of young Peruvian children. Am J } \\
\text { Clin Nutr.2007;85:538-47. }\end{array}$ & IV \\
\hline 82 & $\begin{array}{l}\text { Brown,L. V.,Zeitlin,M. F.,Peterson,K. E.,Chowdhury,A. M.,Rogers,B. L.,Weld,L. H.,Gershoff,S. N. Evaluation of the impact of } \\
\text { weaning food messages on infant feeding practices and child growth in rural Bangladesh. Am J Clin Nutr.1992;56:994-1003. }\end{array}$ & IV \\
\hline 83 & $\begin{array}{l}\text { Brulotte, J.,Bukutu, C.,Vohra, S. Complementary, holistic, and integrative medicine: fish oils and neurodevelopmental disorders. } \\
\text { Pediatr Rev.2009;30:e29-33. }\end{array}$ & Design \\
\hline 84 & $\begin{array}{l}\text { Bulk-Bunschoten, A. M.,van Bodegom, S.,Reerink, J. D.,de Jong, P. C.,de Groot, C. J. Weight and weight gain at } 4 \text { months (The } \\
\text { Netherlands 1998): influences of nutritional practices, socio-economic and ethnic factors. Paediatr Perinat } \\
\text { Epidemiol.2002;16:361-9. }\end{array}$ & IV \\
\hline 85 & $\begin{array}{l}\text { Burnham, L.,Matlak, S.,Makrigiorgos, G.,Braun, N.,Knapp, B. P.,Merewood, A. Breastfeeding and coffee consumption in children } \\
\text { younger than } 2 \text { years in Boston, Massachusetts, USA. J Hum Lact.2015;31:267-72. }\end{array}$ & DV \\
\hline 86 & $\begin{array}{l}\text { Caleyachetty A,Krishnaveni GV,Veena SR,Hill J,Karat SC,Fall CH,Wills AK. Breastfeeding duration, age of starting solids and } \\
\text { high BMI risk and adiposity in Indian children. Matern Child Nutr.2013;9:199-216. }\end{array}$ & $\begin{array}{l}\text { Design,Co } \\
\text { untry }\end{array}$ \\
\hline 87 & Calvo,E. B.,Galindo,A. C.,Aspres,N. B. Iron status in exclusively breast-fed infants. Pediatrics.1992;90:375-9. & IV, DV \\
\hline 88 & $\begin{array}{l}\text { Calvo,E.,Hertrampf,E.,Pablo,S.,Amar,M.,Stekel,A. Haemoglobin-fortified cereal: an alternative weaning food with high iron } \\
\text { bioavailability. European journal of clinical nutrition.1989;43:237-43. }\end{array}$ & $\begin{array}{l}\text { Design, } \\
\text { DV }\end{array}$ \\
\hline 89 & $\begin{array}{l}\text { Cameron, S. L.,Heath, A. L.,Gray, A. R.,Churcher, B.,Davies, R. S.,Newlands, A.,Galland, B. C.,Sayers, R. M.,Lawrence, J. } \\
\text { A.,Taylor, B. J.,Taylor, R. W. Lactation Consultant Support from Late Pregnancy with an Educational Intervention at } 4 \text { Months of } \\
\text { Age Delays the Introduction of Complementary Foods in a Randomized Controlled Trial. J Nutr.2015;145:1481-90. }\end{array}$ & DV \\
\hline 90 & $\begin{array}{l}\text { Capozzi,L.,Russo,R.,Bertocco,F.,Ferrara,D.,Ferrara,M. Diet and iron deficiency in the first year of life: a retrospective study. } \\
\text { Hematology.2010;15:410-3. }\end{array}$ & DV \\
\hline 91 & $\begin{array}{l}\text { Capozzi,L.,Russo,R.,Bertocco,F.,Ferrara,D.,Ferrara,M. Effect on haematological and anthropometric parameters of iron } \\
\text { supplementation in the first } 2 \text { years of life. Risks and benefits. Hematology.2011;16:261-4. }\end{array}$ & IV \\
\hline 92 & $\begin{array}{l}\text { Carruth,B. R.,Nevling,W.,Skinner,J. D. Developmental and food profiles of infants born to adolescent and adult mothers. J } \\
\text { Adolesc Health.1997;20:434-41. }\end{array}$ & $\begin{array}{l}\text { IV, DV, } \\
\text { Age }\end{array}$ \\
\hline
\end{tabular}




\begin{tabular}{|c|c|c|}
\hline 93 & $\begin{array}{l}\text { Casiday, R. E.,Wright, C. M.,Panter-Brick, C.,Parkinson, K. N. Do early infant feeding patterns relate to breast-feeding } \\
\text { continuation and weight gain? Data from a longitudinal cohort study. Eur J Clin Nutr.2004;58:1290-6. }\end{array}$ & IV \\
\hline 94 & $\begin{array}{l}\text { Castillo-Duran,C.,Perales,C. G.,Hertrampf,E. D.,Marin,V. B.,Rivera,F. A.,Icaza,G. Effect of zinc supplementation on development } \\
\text { and growth of Chilean infants. J Pediatr.2001;138:229-35. }\end{array}$ & IV \\
\hline 95 & $\begin{array}{l}\text { Chaimay, B.,Ruagdaraganon, N.,Thinkhamrop, B.,Thinkhamrop, J. Association between infant feeding practices and first } \\
\text { meaningful words at first year of life: a prospective cohort study of Thai children. Asia Pac J Public Health.2015;27:Np1071-84. }\end{array}$ & IV \\
\hline 96 & $\begin{array}{l}\text { Chan,G. M.,Roberts,C. C.,Folland,D.,Jackson,R. Growth and bone mineralization of normal breast-fed infants and the effects of } \\
\text { lactation on maternal bone mineral status. Am J Clin Nutr.1982;36:438-43. }\end{array}$ & IV \\
\hline 97 & $\begin{array}{l}\text { Chang, S. Y.,He, W.,Chen, C. M. Complementary feeding and growth of infant and young child in China. Biomed Environ } \\
\text { Sci.2008;21:264-8. }\end{array}$ & Design \\
\hline 98 & Chatterjee, M. K. Feeding pattern practised by the mothers attending an under five clinic. Indian J Public Health.1987;31:268-9. & Design \\
\hline 99 & Chavez A,Martinez C,Schlaepfer L. Health effects of supplementary feeding programs. Prog Clin Biol Res.1981;67:129-39. & Design \\
\hline 100 & Chawla, P.,Puri, R.,Pershad, D. Impact of pre-school supplementary feeding on mental abilities. Indian Pediatr.1983;20:513-6. & Age \\
\hline 101 & Chawla, P.,Puri, R. Impact of pre-school supplementary feeding programme on anthropometry. Indian Pediatr.1983;20:363-7. & Age \\
\hline 102 & $\begin{array}{l}\text { Cheng, T. S.,Loy, S. L.,Cheung, Y. B.,Chan, J. K.,Pang, W. W.,Godfrey, K. M.,Gluckman, P. D.,Kwek, K.,Saw, S. M.,Chong, Y. } \\
\text { S.,Lee, Y. S.,Lek, N.,Yap, F. Sexually dimorphic response to feeding mode in the growth of infants. Am J Clin } \\
\text { Nutr.2016;103:398-405. }\end{array}$ & IV \\
\hline 103 & $\begin{array}{l}\text { Chirmulay, D.,Nisal, R. Nutritional status of tribal underfive children in Ahmadnagar District, Maharashtra in relation to } \\
\text { weaning/feeding practices. Indian Pediatr.1993;30:215-22. }\end{array}$ & Design \\
\hline 104 & $\begin{array}{l}\text { Chitra, P. Development of banana-based weaning food mixes for infants and its nutritional quality evaluation. Rev Environ } \\
\text { Health.2015;30:125-30. }\end{array}$ & IV \\
\hline 105 & $\begin{array}{l}\text { Chivers, P.,Hands, B.,Parker, H.,Bulsara, M.,Beilin, L. J.,Kendall, G. E.,Oddy, W. H. Body mass index, adiposity rebound and } \\
\text { early feeding in a longitudinal cohort (Raine Study). Int J Obes (Lond).2010;34:1169-76. }\end{array}$ & IV \\
\hline 106 & $\begin{array}{l}\text { Cho, H. N.,Hong, S.,Lee, S. H.,Yum, H. Y. Nutritional status according to sensitized food allergens in children with atopic } \\
\text { dermatitis. Allergy, Asthma and Immunology Research.2010;3:53-57. }\end{array}$ & $\begin{array}{l}\text { Design, } \\
\text { IV, DV }\end{array}$ \\
\hline 107 & $\begin{array}{l}\text { Chorell,E.,Karlsson Videhult,F.,Hernell,O.,Antti,H.,West,C. E. Impact of probiotic feeding during weaning on the serum lipid profile } \\
\text { and plasma metabolome in infants. Br J Nutr.2013;110:116-26. }\end{array}$ & IV \\
\hline 108 & $\begin{array}{l}\text { Choudhury, N.,Bromage, S.,Alam, M. A.,Ahmed, A. M.,Islam, M. M.,Hossain, M. I.,Mahfuz, M.,Mondal, D.,Haque, M. R.,Ahmed, } \\
\text { T. Intervention study shows suboptimal growth among children receiving a food supplement for five months in a slum in } \\
\text { Bangladesh. Acta Paediatr.2016. }\end{array}$ & $\begin{array}{l}\text { Health } \\
\text { status, IV }\end{array}$ \\
\hline 109 & Christoffel, K. A pediatric perspective on vegetarian nutrition. Clin Pediatr (Phila).1981;20:632-43. & Design \\
\hline
\end{tabular}




\begin{tabular}{|c|c|c|}
\hline 110 & Christopher, G. C. First food: the essential role of breastfeeding. Breastfeed Med.2009;4 Suppl 1:S9-s10. & Design \\
\hline 111 & $\begin{array}{l}\text { Chuang, C. H.,Yang, S. H.,Chang, P. J.,Chen, P. C.,Chen, Y. C. Dietary supplement intake by 6-month-old Taiwanese infants. J } \\
\text { Pediatr Gastroenterol Nutr.2012;54:71-6. }\end{array}$ & DV \\
\hline 112 & $\begin{array}{l}\text { Closa-Monasterolo, R.,Gispert-Llaurado, M.,Luque, V.,Ferre, N.,Rubio-Torrents, C.,Zaragoza-Jordana, M.,Escribano, J. Safety } \\
\text { and efficacy of inulin and oligofructose supplementation in infant formula: results from a randomized clinical trial. Clin } \\
\text { Nutr.2013;32:918-27. }\end{array}$ & IV \\
\hline 113 & $\begin{array}{l}\text { Cloutier, M. M.,Wiley, J.,Wang, Z.,Grant, A.,Gorin, A. A. The Early Childhood Obesity Prevention Program (ECHO): an } \\
\text { ecologically-based intervention delivered by home visitors for newborns and their mothers. BMC Public Health.2015;15:584. }\end{array}$ & Design \\
\hline 114 & Cockburn,F. Neonatal brain and dietary lipids. Arch Dis Child Fetal Neonatal Ed.1994;70:F1-2. & Design \\
\hline 115 & $\begin{array}{l}\text { Cohen RJ,Brown KH,Canahuati J,Rivera LL,Dewey KG. Effects of age of introduction of complementary foods on infant breast } \\
\text { milk intake, total energy intake, and growth: a randomised intervention study in Honduras. Lancet.1994;344:288-93. }\end{array}$ & Country \\
\hline 116 & $\begin{array}{l}\text { Cohen, R. J.,Brown, K. H.,Canahuati, J.,Rivera, L. L.,Dewey, K. G. Determinants of growth from birth to } 12 \text { months among } \\
\text { breast-fed Honduran infants in relation to age of introduction of complementary foods. Pediatrics.1995;96:504-10. }\end{array}$ & IV \\
\hline 117 & Coleman,B. L. Early introduction of non-formula cow's milk to southern Ontario infants. Can J Public Health.2006;97:187-90. & IV, DV \\
\hline 118 & $\begin{array}{l}\text { Colombo,J.,Carlson,S. E.,Cheatham,C. L.,Shaddy,D. J.,Kerling,E. H.,Thodosoff,J. M.,Gustafson,K. M.,Brez,C. Long-term effects } \\
\text { of LCPUFA supplementation on childhood cognitive outcomes. Am J Clin Nutr.2013;98:403-12. }\end{array}$ & IV \\
\hline 119 & $\begin{array}{l}\text { Coppi, S., lacoponi, F.,Fommei, C.,Strambi, M. Growth trend during the first six months of life in male infants with different type of } \\
\text { feeding. Minerva Pediatr.2013;65:51-9. }\end{array}$ & IV \\
\hline 120 & $\begin{array}{l}\text { Costom, B. H.,Shore, D. Effect of a comprehensive nutritional program on the growth and ponderosity of infants. Clin Pediatr } \\
\text { (Phila).1983;22:105-11. }\end{array}$ & Design \\
\hline 121 & $\begin{array}{l}\text { Courage, M. L.,McCloy, U. R.,Herzberg, G. R.,Andrews, W. L.,Simmons, B. S.,McDonald, A. C.,Mercer, C. N.,Friel, J. K. Visual } \\
\text { acuity development and fatty acid composition of erythrocytes in full-term infants fed breast milk, commercial formula, or } \\
\text { evaporated milk. J Dev Behav Pediatr.1998;19:9-17. }\end{array}$ & IV \\
\hline 122 & Cusack, R. Dietary management of obese children and adolescents. Pediatr Ann.1984;13:455, 458-9, 462-4. & Design \\
\hline 123 & $\begin{array}{l}\text { Dagan, R.,Sofer, S.,Klish, W. J.,Hundt, G.,Saltz-Rennert, H.,Moses, S. W. Infant feeding practices among Bedouins in transition } \\
\text { from seminomadic to settlement conditions in the Negev area of Israel. Isr J Med Sci.1984;20:1029-34. }\end{array}$ & $\begin{array}{l}\text { Design, } \\
\text { DV }\end{array}$ \\
\hline 124 & $\begin{array}{l}\text { Dagnelie,P. C.,van Staveren,W. A.,Hautvast,J. G. Stunting and nutrient deficiencies in children on alternative diets. Acta Paediatr } \\
\text { Scand Suppl.1991;374:111-8. }\end{array}$ & DV \\
\hline 125 & $\begin{array}{l}\text { Dagnelie,P. C.,van Staveren,W. A.,Vergote,F. J.,Dingjan,P. G.,van den Berg,H.,Hautvast,J. G. Increased risk of vitamin B-12 and } \\
\text { iron deficiency in infants on macrobiotic diets. Am J Clin Nutr.1989;50:818-24. }\end{array}$ & $\begin{array}{l}\text { Design, } \\
\text { DV }\end{array}$ \\
\hline 126 & $\begin{array}{l}\text { Dagnelie,P. C.,van Staveren,W. A.,Verschuren,S. A.,Hautvast,J. G. Nutritional status of infants aged } 4 \text { to } 18 \text { months on } \\
\text { macrobiotic diets and matched omnivorous control infants: a population-based mixed-longitudinal study. I. Weaning pattern, }\end{array}$ & $\begin{array}{l}\text { Design, } \\
\text { DV }\end{array}$ \\
\hline
\end{tabular}




\begin{tabular}{|c|c|c|}
\hline & energy and nutrient intake. Eur J Clin Nutr.1989;43:311-23. & \\
\hline 127 & $\begin{array}{l}\text { Daniels, L. A.,Mallan, K. M.,Battistutta, D.,Nicholson, J. M.,Meedeniya, J. E.,Bayer, J. K.,Magarey, A. Child eating behavior } \\
\text { outcomes of an early feeding intervention to reduce risk indicators for child obesity: the NOURISH RCT. Obesity (Silver } \\
\text { Spring).2014;22:E104-11. }\end{array}$ & DV \\
\hline 128 & $\begin{array}{l}\text { Daniels, L. A., Mallan, K. M.,Battistutta, D.,Nicholson, J. M.,Perry, R.,Magarey, A. Evaluation of an intervention to promote } \\
\text { protective infant feeding practices to prevent childhood obesity: outcomes of the NOURISH RCT at } 14 \text { months of age and } 6 \\
\text { months post the first of two intervention modules. Int J Obes (Lond).2012;36:1292-8. }\end{array}$ & IV, DV \\
\hline 129 & $\begin{array}{l}\text { Daniels, L. A.,Mallan, K. M.,Nicholson, J. M.,Battistutta, D.,Magarey, A. Outcomes of an early feeding practices intervention to } \\
\text { prevent childhood obesity. Pediatrics.2013;132:e109-18. }\end{array}$ & IV \\
\hline 130 & $\begin{array}{l}\text { Daniels, L. A.,Mallan, K. M.,Nicholson, J. M.,Thorpe, K.,Nambiar, S.,Mauch, C. E.,Magarey, A. An Early Feeding Practices } \\
\text { Intervention for Obesity Prevention. Pediatrics.2015;136:e40-9. }\end{array}$ & IV \\
\hline 131 & Daniels, M. C.,Adair, L. S. Breast-feeding influences cognitive development in Filipino children. J Nutr.2005;135:2589-95. & IV \\
\hline 132 & $\begin{array}{l}\text { Davidsson,L.,Sarker,S. A.,Jamil,K. A.,Sultana,S.,Hurrell,R. Regular consumption of a complementary food fortified with ascorbic } \\
\text { acid and ferrous fumarate or ferric pyrophosphate is as useful as ferrous sulfate in maintaining hemoglobin concentrations }>105 \\
\text { g/L in young Bangladeshi children. Am J Clin Nutr.2009;89:1815-20. }\end{array}$ & IV, DV \\
\hline 133 & $\begin{array}{l}\text { de Hoog ML,van Eijsden M,Stronks K,Gemke RJ,Vrijkotte TG. The role of infant feeding practices in the explanation for ethnic } \\
\text { differences in infant growth: the Amsterdam Born Children and their Development study. Br J Nutr.2011;106:1592-601. }\end{array}$ & Design \\
\hline 134 & $\begin{array}{l}\text { de Kanashiro, H. C.,Brown, K. H.,Lopez de Romana, G.,Lopez, T.,Black, R. E. Consumption of food and nutrients by infants in } \\
\text { Huascar (Lima), Peru. Am J Clin Nutr.1990;52:995-1004. }\end{array}$ & DV \\
\hline 135 & $\begin{array}{l}\text { de Villiers, A.,Senekal, M. Determinants of growth failure in 12-24-month-old children in a high-density urban slum community in } \\
\text { East London, South Africa. Eur J Clin Nutr.2002;56:1231-41. }\end{array}$ & Country \\
\hline 136 & $\begin{array}{l}\text { de Zegher, F.,Sebastiani, G.,Diaz, M.,Sanchez-Infantes, D.,Lopez-Bermejo, A.,Ibanez, L. Body composition and circulating high- } \\
\text { molecular-weight adiponectin and IGF-I in infants born small for gestational age: breast- versus formula-feeding. } \\
\text { Diabetes.2012;61:1969-73. }\end{array}$ & $\begin{array}{l}\text { Health } \\
\text { status, IV }\end{array}$ \\
\hline 137 & Delport, S. D.,Becker, P. J.,Bergh, A. Growth, feeding practices and infections in black infants. S Afr Med J.1997;87:57-61. & IV, DV \\
\hline 138 & $\begin{array}{l}\text { Dewey, K. G.,Cohen, R. J.,Rivera, L. L.,Canahuati, J.,Brown, K. H. Do exclusively breast-fed infants require extra protein?. } \\
\text { Pediatr Res.1996;39:303-7. }\end{array}$ & IV \\
\hline 139 & $\begin{array}{l}\text { Dewey, K. G.,Hawck, M. G.,Brown, K. H.,Lartey, A.,Cohen, R. J.,Peerson, J. M. Infant weight-for-length is positively associated } \\
\text { with subsequent linear growth across four different populations. Matern Child Nutr.2005;1:11-20. }\end{array}$ & IV \\
\hline 140 & $\begin{array}{l}\text { Dewey, K. G.,Heinig, M. J.,Nommsen, L. A.,Lonnerdal, B. Adequacy of energy intake among breast-fed infants in the DARLING } \\
\text { study: relationships to growth velocity, morbidity, and activity levels. Davis Area Research on Lactation, Infant Nutrition and } \\
\text { Growth. J Pediatr.1991;119:538-47. }\end{array}$ & IV \\
\hline
\end{tabular}




\begin{tabular}{|c|c|c|}
\hline 141 & $\begin{array}{l}\text { Dewey, K. G.,Lonnerdal, B. Milk and nutrient intake of breast-fed infants from } 1 \text { to } 6 \text { months: relation to growth and fatness. J } \\
\text { Pediatr Gastroenterol Nutr.1983;2:497-506. }\end{array}$ & IV \\
\hline 142 & $\begin{array}{l}\text { Dewey, K. G.,Peerson, J. M.,Brown, K. H.,Krebs, N. F.,Michaelsen, K. F.,Persson, L. A.,Salmenpera, L.,Whitehead, R. G.,Yeung, } \\
\text { D. L. Growth of breast-fed infants deviates from current reference data: a pooled analysis of US, Canadian, and European data } \\
\text { sets. World Health Organization Working Group on Infant Growth. Pediatrics.1995;96:495-503. }\end{array}$ & Design \\
\hline 143 & $\begin{array}{l}\text { Dewey, K. G.,Peerson, J. M.,Heinig, M. J.,Nommsen, L. A.,Lonnerdal, B.,Lopez de Romana, G., de Kanashiro, H. C.,Black, R. } \\
\text { E.,Brown, K. H. Growth patterns of breast-fed infants in affluent (United States) and poor (Peru) communities: implications for } \\
\text { timing of complementary feeding. Am J Clin Nutr.1992;56:1012-8. }\end{array}$ & IV, DV \\
\hline 144 & Dewey, K. G. Complementary feeding and infant growth and body composition. Pediatrics.2000;106:1281. & Design \\
\hline 145 & $\begin{array}{l}\text { Dewey,K. G.,Cohen,R. J.,Brown,K. H.,Rivera,L. L. Effects of exclusive breastfeeding for four versus six months on maternal } \\
\text { nutritional status and infant motor development: results of two randomized trials in Honduras. J Nutr.2001;131:262-7. }\end{array}$ & Country \\
\hline 146 & $\begin{array}{l}\text { Dewey,K. G.,Cohen,R. J.,Rivera,L. L.,Brown,K. H. Effects of age of introduction of complementary foods on iron status of breast- } \\
\text { fed infants in Honduras. Am J Clin Nutr.1998;67:878-84. }\end{array}$ & DV \\
\hline 147 & $\begin{array}{l}\text { Dewey,K. G.,Huffman,S. L. Maternal, infant, and young child nutrition: combining efforts to maximize impacts on child growth and } \\
\text { micronutrient status. Food Nutr Bull.2009;30:S187-9. }\end{array}$ & Design \\
\hline 148 & $\begin{array}{l}\text { Dewey,K. G.,Romero-Abal,M. E.,Quan de Serrano,J.,Bulux,J.,Peerson,J. M.,Eagle,P.,Solomons,N. W. Effects of discontinuing } \\
\text { coffee intake on iron status of iron-deficient Guatemalan toddlers: a randomized intervention study. Am J Clin Nutr.1997;66:168- } \\
76 .\end{array}$ & DV \\
\hline 149 & $\begin{array}{l}\text { Diaz, S.,Herreros, C.,Aravena, R.,Casado, M. E.,Reyes, M. V.,Schiappacasse, V. Breast-feeding duration and growth of fully } \\
\text { breast-fed infants in a poor urban Chilean population. Am J Clin Nutr.1995;62:371-6. }\end{array}$ & IV, DV \\
\hline 150 & $\begin{array}{l}\text { Doak, C. M.,van der Starre, R. E.,van Beusekom, I.,Campos Ponce, M., Vossenaar, M.,Solomons, N. W. Earlier introduction of } \\
\text { aguitas is associated with higher risk of stunting in infants and toddlers in the Western Highlands of Guatemala. Am J Clin } \\
\text { Nutr.2013;97:631-6. }\end{array}$ & Design \\
\hline 151 & $\begin{array}{l}\text { Domellof,M.,Cohen,R. J.,Dewey,K. G.,Hernell,O.,Rivera,L. L.,Lonnerdal,B. Iron supplementation of breast-fed Honduran and } \\
\text { Swedish infants from } 4 \text { to } 9 \text { months of age. J Pediatr.2001;138:679-87. }\end{array}$ & IV, DV \\
\hline 152 & $\begin{array}{l}\text { Dong,C.,Ge,P.,Ren,X.,Wang,J.,Fan,H.,Yan,X.,Yin,S. A. Prospective study on the effectiveness of complementary food } \\
\text { supplements on improving status of elder infants and young children in the areas affected by Wenchuan earthquake. PLoS } \\
\text { One.2013;8:e72711. }\end{array}$ & $\begin{array}{l}\text { Design, } \\
\text { Health } \\
\text { status }\end{array}$ \\
\hline 153 & Donma, M. M.,Donma, O. Infant feeding and growth: a study on Turkish infants from birth to 6 months. Pediatr Int.1999;41:542-8. & IV \\
\hline 154 & $\begin{array}{l}\text { Donma, M. M.,Donma, O. The influence of feeding patterns on head circumference among Turkish infants during the first } 6 \\
\text { months of life. Brain Dev.1997;19:393-7. }\end{array}$ & IV \\
\hline 155 & $\begin{array}{l}\text { Dubakiene, R.,Rudzeviciene, O.,Butiene, I.,Sezaite, I.,Petronyte, M.,Vaicekauskaite, D.,Zvirbliene, A. Studies on early allergic } \\
\text { sensitization in the Lithuanian birth cohort. ScientificWorldJournal.2012;2012:909524. }\end{array}$ & IV, DV \\
\hline
\end{tabular}




\begin{tabular}{|c|c|c|}
\hline 156 & $\begin{array}{l}\text { Dubois, L.,Girard, M. Breast-feeding, day-care attendance and the frequency of antibiotic treatments from } 1.5 \text { to } 5 \text { years: a } \\
\text { population-based longitudinal study in Canada. Soc Sci Med.2005;60:2035-44. }\end{array}$ & IV, DV \\
\hline 157 & $\begin{array}{l}\text { Dubois, L., Girard, M. Social inequalities in infant feeding during the first year of life. The Longitudinal Study of Child Development } \\
\text { in Quebec (LSCDQ 1998-2002). Public Health Nutr.2003;6:773-83. }\end{array}$ & IV, DV \\
\hline 158 & $\begin{array}{l}\text { Dubowitz, T.,Levinson, D.,Peterman, J. N.,Verma, G.,Jacob, S.,Schultink, W. Intensifying efforts to reduce child malnutrition in } \\
\text { India: an evaluation of the Dular program in Jharkhand, India. Food Nutr Bull.2007;28:266-73. }\end{array}$ & IV \\
\hline 159 & $\begin{array}{l}\text { Duggan,C.,Penny,M. E.,Hibberd,P.,Gil,A.,Huapaya,A.,Cooper,A.,Coletta,F.,Emenhiser,C.,Kleinman,R. E. Oligofructose- } \\
\text { supplemented infant cereal: } 2 \text { randomized, blinded, community-based trials in Peruvian infants. Am J Clin Nutr.2003;77:937-42. }\end{array}$ & IV \\
\hline 160 & Dunne,A. Nutrition in infancy: achieving nutrition needs for new mothers and children. Br J Community Nurs.2012;Suppl:S22. & Design \\
\hline 161 & Edmondson, L. What toddlers eat really matters. J Fam Health Care.2011;21:33-41. & Design \\
\hline 162 & $\begin{array}{l}\text { El-Sayed H,Martines J,Rakha M,Zekry O,Abdel-Hak M,Abbas H. The effectiveness of the WHO training course on } \\
\text { complementary feeding counseling in a primary care setting, Ismailia, Egypt. J Egypt Public Health Assoc.2014;89:1-8. }\end{array}$ & IV \\
\hline 163 & $\begin{array}{l}\text { Elwood, P. C., Haley, T. J.,Hughes, S. J.,Sweetnam, P. M.,Gray, O. P.,Davies, D. P. Child growth (0-5 years), and the effect of } \\
\text { entitlement to a milk supplement. Arch Dis Child.1981;56:831-5. }\end{array}$ & IV \\
\hline 164 & $\begin{array}{l}\text { Emmett,P. M.,Jones,L. R. Diet and growth in infancy: relationship to socioeconomic background and to health and development } \\
\text { in the Avon Longitudinal Study of Parents and Children. Nutr Rev.2014;72:483-506. }\end{array}$ & Design \\
\hline 165 & $\begin{array}{l}\text { Emond A,Drewett R,Blair P,Emmett P. Postnatal factors associated with failure to thrive in term infants in the Avon Longitudinal } \\
\text { Study of Parents and Children. Arch Dis Child.2007;92:115-9. }\end{array}$ & IV \\
\hline 166 & $\begin{array}{l}\text { Eneroth,H.,El Arifeen,S.,Persson,L. A.,Kabir,I.,Lonnerdal,B.,Hossain,M. B.,Ekstrom,E. C. Duration of exclusive breast-feeding } \\
\text { and infant iron and zinc status in rural Bangladesh. J Nutr.2009;139:1562-7. }\end{array}$ & IV, DV \\
\hline 167 & $\begin{array}{l}\text { Engelmann,M. D.,Davidsson,L.,Sandstrom,B.,Walczyk,T.,Hurrell,R. F.,Michaelsen,K. F. The influence of meat on nonheme iron } \\
\text { absorption in infants. Pediatr Res.1998;43:768-73. }\end{array}$ & DV \\
\hline 168 & $\begin{array}{l}\text { England, L.,Brenner, R.,Bhaskar, B.,Simons-Morton, B.,Das, A., Revenis, M.,Mehta, N.,Clemens, J. Breastfeeding practices in a } \\
\text { cohort of inner-city women: the role of contraindications. BMC Public Health.2003;3:28. }\end{array}$ & IV, DV \\
\hline 169 & $\begin{array}{l}\text { Escribano, J.,Luque, V.,Ferre, N.,Mendez-Riera, G.,Koletzko, B.,Grote, V.,Demmelmair, H.,Bluck, L.,Wright, A.,Closa- } \\
\text { Monasterolo, R. Effect of protein intake and weight gain velocity on body fat mass at } 6 \text { months of age: the EU Childhood Obesity } \\
\text { Programme. Int J Obes (Lond).2012;36:548-53. }\end{array}$ & IV \\
\hline 170 & $\begin{array}{l}\text { Esfarjani, F.,Azar, M. R.,Gafarpour, M. IDDM and early exposure of infant to cow's milk and solid food. Indian J } \\
\text { Pediatr.2001;68:107-10. }\end{array}$ & DV \\
\hline 171 & Evans, D.,Hansen, J. D.,Moodie, A. D.,van der Spuy, H. I. Intellectual development and nutrition. J Pediatr.1980;97:358-63. & $\begin{array}{l}\text { Health } \\
\text { status, IV }\end{array}$ \\
\hline 172 & $\begin{array}{l}\text { Exl, B. M.,Deland, U.,Secretin, M. C.,Preysch, U.,Wall, M.,Shmerling, D. H. Improved general health status in an unselected } \\
\text { infant population following an allergen reduced dietary intervention programme. The ZUFF-study-programme. Part I: Study design }\end{array}$ & DV \\
\hline
\end{tabular}




\begin{tabular}{|c|c|c|}
\hline & and 6-month nutritional behaviour. Eur J Nutr.2000;39:89-102. & \\
\hline 173 & $\begin{array}{l}\text { Exl, B. M.,Deland, U.,Secretin, M. C.,Preysch, U.,Wall, M.,Shmerling, D. H. Improved general health status in an unselected } \\
\text { infant population following an allergen-reduced dietary intervention programme: the ZUFF-STUDY-PROGRAMME. Part II: infant } \\
\text { growth and health status to age } 6 \text { months. ZUg-FrauenFeld. Eur J Nutr.2000;39:145-56. }\end{array}$ & IV \\
\hline 174 & $\begin{array}{l}\text { Faber,M.,Kvalsvig,J. D.,Lombard,C. J.,Benade,A. J. Effect of a fortified maize-meal porridge on anemia, micronutrient status, and } \\
\text { motor development of infants. Am J Clin Nutr.2005;82:1032-9. }\end{array}$ & Country \\
\hline 175 & $\begin{array}{l}\text { Faber,M. Dietary intake and anthropometric status differ for anaemic and non-anaemic rural South African infants aged 6-12 } \\
\text { months. J Health Popul Nutr.2007;25:285-93. }\end{array}$ & Design \\
\hline 176 & $\begin{array}{l}\text { Fabiansen, C.,Phelan, K. P.,Cichon, B.,Ritz, C.,Briend, A.,Michaelsen, K. F.,Friis, H.,Shepherd, S. Short children with a low } \\
\text { midupper arm circumference respond to food supplementation: an observational study from Burkina Faso. Am J Clin } \\
\text { Nutr.2016;103:415-21. }\end{array}$ & $\begin{array}{l}\text { Health } \\
\text { status }\end{array}$ \\
\hline 177 & $\begin{array}{l}\text { Fabius, R. J.,Merritt, R. J.,Fleiss, P. M.,Ashley, J. M. Malnutrition associated with a formula of barley water, corn syrup, and whole } \\
\text { milk. Am J Dis Child.1981;135:615-7. }\end{array}$ & Design \\
\hline 178 & Falkner, F. Introduction of food to infants. Genetic-environmental considerations. Am J Clin Nutr.1985;41:436-9. & Design \\
\hline 179 & $\begin{array}{l}\text { Fall } \mathrm{CH} \text {,Borja JB,Osmond C,Richter L,Bhargava SK,Martorell R,Stein AD,Barros FC,Victora CG. Infant-feeding patterns and } \\
\text { cardiovascular risk factors in young adulthood: data from five cohorts in low- and middle-income countries. Int } \mathrm{J} \\
\text { Epidemiol.2011;40:47-62. }\end{array}$ & Design \\
\hline 180 & $\begin{array}{l}\text { Farris, R. P.,Frank, G. C., Webber, L. S.,Srinivasan, S. R.,Berenson, G. S. Influence of milk source on serum lipids and } \\
\text { lipoproteins during the first year of life, Bogalusa heart study. Am J Clin Nutr.1982;35:42-9. }\end{array}$ & IV \\
\hline 181 & Farrow, C. V.,Blissett, J. Controlling feeding practices: cause or consequence of early child weight?. Pediatrics.2008;121:e164-9. & IV \\
\hline 182 & Farrow, C.,Blissett, J. Does maternal control during feeding moderate early infant weight gain?. Pediatrics.2006;118:e293-8. & IV \\
\hline 183 & Fawcett, J. N. Feeding from birth to 18 months. Nursing (Lond).1981:956-8. & Design \\
\hline 184 & $\begin{array}{l}\text { Feldens CA,Vitolo MR, Rauber F,Cruz LN,Hilgert JB. Risk factors for discontinuing breastfeeding in southern Brazil: a survival } \\
\text { analysis. Matern Child Health J.2012;16:1257-65. }\end{array}$ & IV, DV \\
\hline 185 & $\begin{array}{l}\text { Feldens, C. A.,Ardenghi, T. M.,Cruz, L. N.,Scalco, G. P.,Vitolo, M. R. Advising mothers about breastfeeding and weaning reduced } \\
\text { pacifier use in the first year of life: a randomized trial. Community Dent Oral Epidemiol.2013;41:317-26. }\end{array}$ & IV, DV \\
\hline 186 & $\begin{array}{l}\text { Feldens, C. A.,Kramer, P. F.,Sequeira, M. C.,Rodrigues, P. H.,Vitolo, M. R. Maternal education is an independent determinant of } \\
\text { cariogenic feeding practices in the first year of life. Eur Arch Paediatr Dent.2012;13:70-5. }\end{array}$ & DV \\
\hline 187 & $\begin{array}{l}\text { Fergusson, D. M.,Woodward, L. J. Breast feeding and later psychosocial adjustment. Paediatr Perinat Epidemiol.1999;13:144- } \\
57 .\end{array}$ & IV \\
\hline 188 & Fernandes, T. A.,Werneck, G. L.,Hasselmann, M. H. Prepregnancy weight, weight gain during pregnancy, and exclusive & IV, DV \\
\hline
\end{tabular}




\begin{tabular}{|c|c|c|}
\hline & breastfeeding in the first month of Life in Rio de Janeiro, Brazil. J Hum Lact.2012;28:55- & \\
\hline 189 & $\begin{array}{l}\text { Fernstrom, J. D.,Navia, J. L. Introduction to the workshop. Low-calorie sweeteners and weight control--what the science tells us. } \\
\text { J Nutr.2012;142:1132s-3s. }\end{array}$ & Design \\
\hline 190 & $\begin{array}{l}\text { Ferrara,M.,Bertocco,F.,Ricciardi,A.,Ferrara,D.,Incarnato,L.,Capozzi,L. Iron deficiency screening in the first three years of life: a } \\
\text { three-decade-long retrospective case study. Hematology.2014;19:239-43. }\end{array}$ & DV \\
\hline 191 & $\begin{array}{l}\text { Fildes, A.,van Jaarsveld, C. H.,Llewellyn, C.,Wardle, J.,Fisher, A. Parental control over feeding in infancy. Influence of infant } \\
\text { weight, appetite and feeding method. Appetite.2015;91:101-6. }\end{array}$ & IV, DV \\
\hline 192 & $\begin{array}{l}\text { Fisher, J. O.,Birch, L. L.,Smiciklas-Wright, H.,Picciano, M. F. Breast-feeding through the first year predicts maternal control in } \\
\text { feeding and subsequent toddler energy intakes. J Am Diet Assoc.2000;100:641-6. }\end{array}$ & IV, DV \\
\hline 193 & $\begin{array}{l}\text { Flax, V. L.,Bentley, M. E.,Chasela, C. S.,Kayira, D.,Hudgens, M. G.,Kacheche, K. Z.,Chavula, C.,Kourtis, A. P.,Jamieson, D. } \\
\text { J.,van der Horst, C. M.,Adair, L. S. Lipid-based nutrient supplements are feasible as a breastmilk replacement for HIV-exposed } \\
\text { infants from } 24 \text { to } 48 \text { weeks of age. J Nutr.2013;143:701-7. }\end{array}$ & $\begin{array}{l}\text { Health } \\
\text { status, IV }\end{array}$ \\
\hline 194 & $\begin{array}{l}\text { Fomon SJ,Ziegler EE,Nelson SE,Edwards BB. Sweetness of diet and food consumption by infants. Proc Soc Exp Biol } \\
\text { Med.1983;173:190-3. }\end{array}$ & IV, DV \\
\hline 195 & $\begin{array}{l}\text { Fomon, S. J.,Rogers, R. R.,Ziegler, E. E.,Nelson, S. E.,Thomas, L. N. Indices of fatness and serum cholesterol at age eight years } \\
\text { in relation to feeding and growth during early infancy. Pediatr Res.1984;18:1233-8. }\end{array}$ & IV, Date \\
\hline 196 & $\begin{array}{l}\text { Forns,J.,Torrent,M.,Garcia-Esteban,R.,Caceres,A.,Pilar Gomila,M.,Martinez,D.,Morales,E.,Julvez,J.,Grimalt,J. O.,Sunyer,J. } \\
\text { Longitudinal association between early life socio-environmental factors and attention function at the age } 11 \text { years. Environ } \\
\text { Res.2012;117:54-9. }\end{array}$ & IV \\
\hline 197 & $\begin{array}{l}\text { Foroushani, A. R.,Mohammad, K.,Mahmoodi, M.,Siassi, F. Effect of breastfeeding on cognitive performance in a British birth } \\
\text { cohort. East Mediterr Health J.2010;16:202-8. }\end{array}$ & IV \\
\hline 198 & $\begin{array}{l}\text { Franks, A. J.,Jurgensen, C. Nutrition and health in the first year of life on a Pacific atoll. Observations on Abemama Atoll, Central } \\
\text { Pacific. Trans R Soc Trop Med Hyg.1985;79:681-4. }\end{array}$ & IV, DV \\
\hline 199 & $\begin{array}{l}\text { Frederiksen,B. N.,Seifert,J.,Kroehl,M.,Lamb,M. M.,Milne,G. L.,Rewers,M.,Norris,J. M. Timing of solid food introduction is } \\
\text { associated with urinary F2-isoprostane concentrations in childhood. Pediatric Research.2015;78:451-456. }\end{array}$ & DV \\
\hline 200 & $\begin{array}{l}\text { Freeman, V.,van't Hof, M.,Haschke, F. Patterns of milk and food intake in infants from birth to age } 36 \text { months: the Euro-growth } \\
\text { study. J Pediatr Gastroenterol Nutr.2000;31 Suppl 1:S76-85. }\end{array}$ & DV \\
\hline 201 & $\begin{array}{l}\text { Freeman,V. E.,Mulder,J.,van't Hof,M. A.,Hoey,H. M.,Gibney,M. J. A longitudinal study of iron status in children at } 12,24 \text { and } 36 \\
\text { months. Public Health Nutr.1998;1:93-100. }\end{array}$ & DV \\
\hline 202 & $\begin{array}{l}\text { Friel,J. K.,Andrews,W. L.,Simmons,B. S.,L'Abbe,M. R.,Mercer,C.,MacDonald,A.,McCloy,U. R. Evaluation of full-term infants fed } \\
\text { an evaporated milk formula. Acta Paediatr.1997;86:448-53. }\end{array}$ & IV \\
\hline 203 & $\begin{array}{l}\text { Froozani, M. D.,Malekafzali, H.,Bahrini, B. Growth of a group of low income infants in the first year of life. J Trop } \\
\text { Pediatr.1980;26:96-8. }\end{array}$ & Design, IV \\
\hline
\end{tabular}




\begin{tabular}{|c|c|c|}
\hline 204 & $\begin{array}{l}\text { Fuchs lii, G. J.,Gastanaduy, A. S.,Suskind, R. M. Comparative metabolic study of older infants fed infant formula, transition } \\
\text { formula, or whole cow's milk. Nutrition Research.1992;12:1467-1478. }\end{array}$ & IV \\
\hline 205 & $\begin{array}{l}\text { Fuchs, G. J.,Farris, R. P.,DeWier, M.,Hutchinson, S.,Strada, R.,Suskind, R. M. Effect of dietary fat on cardiovascular risk factors } \\
\text { in infancy. Pediatrics.1994;93:756-63. }\end{array}$ & IV \\
\hline 206 & $\begin{array}{l}\text { Gabriel, C. G.,Corso, A. C.,Caldeira, G. V.,Gimeno, S. G.,Schmitz Bde, A.,de Vasconcelos Fde, A. Overweight and obesity } \\
\text { related factors in schoolchildren in Santa Catarina State, Brazil. Arch Latinoam Nutr.2010;60:332-9. }\end{array}$ & Design \\
\hline 207 & $\begin{array}{l}\text { Gaffney, K. F.,Brito, A. V.,Kitsantas, P.,Kermer, D. A. Early Feeding Practices and Weight Status at One Year of Age: A } \\
\text { Comparison of Hispanic Immigrant Mother-Infant Dyads with Participants of the Infant Feeding Practices Study II. Child } \\
\text { Obes.2016. }\end{array}$ & IV, DV \\
\hline 208 & $\begin{array}{l}\text { Gale, C.,Thomas, E. L.,Jeffries, S.,Durighel, G.,Logan, K. M.,Parkinson, J. R.,Uthaya, S.,Santhakumaran, S.,Bell, J. D.,Modi, N. } \\
\text { Adiposity and hepatic lipid in healthy full-term, breastfed, and formula-fed human infants: a prospective short-term longitudinal } \\
\text { cohort study. Am J Clin Nutr.2014;99:1034-40. }\end{array}$ & IV \\
\hline 209 & $\begin{array}{l}\text { Galler, J. R.,Ramsey, F. C.,Harrison, R. H.,Brooks, R.,Weiskopf-Bock, S. Infant feeding practices in Barbados predict later } \\
\text { growth. J Nutr.1998;128:1328-35. }\end{array}$ & IV \\
\hline 210 & $\begin{array}{l}\text { Gallo PV. Physiological and behavioral consequences of maternal and post-weaning protein restriction. Physiol } \\
\text { Behav.1981;26:77-84. }\end{array}$ & $\begin{array}{l}\text { Design, } \\
\text { Health } \\
\text { status }\end{array}$ \\
\hline 211 & $\begin{array}{l}\text { Galtry, J. Punching above its weight: does New Zealand's responsibility for protecting, promoting, and supporting breastfeeding } \\
\text { extend beyond its own borders?. J Hum Lact.2013;29:128-31. }\end{array}$ & Design \\
\hline 212 & $\begin{array}{l}\text { Garza, C. The INTERGROWTH-21st project and the multicenter growth reference study: enhanced opportunities for monitoring } \\
\text { growth from early pregnancy to } 5 \text { years of age. Breastfeed Med.2014;9:341-4. }\end{array}$ & Design \\
\hline 213 & $\begin{array}{l}\text { Geuns, M.,Huisinga, C.,van Staveren, W. A.,Deurenberg, P.,Hautvast, J. G. Intakes of energy, protein and calcium and weaning } \\
\text { pattern of a group of Dutch infants aged 6-12 months. Tijdschr Kindergeneeskd.1985;53:50-6. }\end{array}$ & $\begin{array}{l}\text { Design, IV, } \\
\text { DV }\end{array}$ \\
\hline 214 & $\begin{array}{l}\text { Ghorashi,Z.,Nezami,N.,Ghalehgolab Behbahan,A.,Ghorashi,S. Supplemental food may not prevent iron-deficiency anemia in } \\
\text { infants. Indian J Pediatr.2008;75:1121-4. }\end{array}$ & DV \\
\hline 215 & $\begin{array}{l}\text { Ghosh,S.,Kilaru,A., Ganapathy,S. Nutrition education and infant growth in rural Indian infants: narrowing the gender gap?. J } \\
\text { Indian Med Assoc.2002;100:483-4, 486-8, 490. }\end{array}$ & IV \\
\hline 216 & $\begin{array}{l}\text { Gianni, M. L.,Roggero, P.,Morlacchi, L.,Garavaglia, E.,Piemontese, P.,Mosca, F. Formula-fed infants have significantly higher fat- } \\
\text { free mass content in their bodies than breastfed babies. Acta Paediatr.2014;103:e277-81. }\end{array}$ & IV \\
\hline 217 & Gibson,R. S.,Hotz,C. The adequacy of micronutrients in complementary foods. Pediatrics.2000;106:1298-9. & Design \\
\hline 218 & $\begin{array}{l}\text { Gill, D. G.,Vincent, S.,Segal, D. S. Follow-on formula in the prevention of iron deficiency: a multicentre study. Acta } \\
\text { Paediatr.1997;86:683-9. }\end{array}$ & IV \\
\hline 219 & $\begin{array}{l}\text { Gillman, M. W.,Rifas-Shiman, S. L.,Camargo, C. A., Jr.,Berkey, C. S.,Frazier, A. L.,Rockett, H. R.,Field, A. E.,Colditz, G. A. Risk } \\
\text { of overweight among adolescents who were breastfed as infants. Jama.2001;285:2461-7. }\end{array}$ & Design \\
\hline
\end{tabular}




\begin{tabular}{|c|c|c|}
\hline 220 & $\begin{array}{l}\text { Gokcay, G.,Turan, J. M.,Partalci, A.,Neyzi, O. Growth of infants during the first year of life according to feeding regimen in the first } \\
4 \text { months. J Trop Pediatr.2003;49:6-12. }\end{array}$ & IV \\
\hline 221 & $\begin{array}{l}\text { Golley,R. K.,Smithers,L. G.,Mittinty,M. N.,Brazionis,L.,Emmett,P.,Northstone,K.,Campbell,K.,McNaughton,S. A.,Lynch,J. W. An } \\
\text { index measuring adherence to complementary feeding guidelines has convergent validity as a measure of infant diet quality. J } \\
\text { Nutr.2012;142:901-8. }\end{array}$ & IV, DV \\
\hline 222 & $\begin{array}{l}\text { Gomez Santos, S. F.,Estevez Santiago, R.,Palacios Gil-Antunano, N.,Leis Trabazo, M. R.,Tojo Sierra, R.,Cuadrado Vives, } \\
\text { C.,Beltran de Miguel, B.,Avila Torres, J. M.,Varela Moreiras, G.,Casas Esteve, R. THAO-CHILD HEALTH PROGRAMME: } \\
\text { COMMUNITY BASED INTERVENTION FOR HEALTHY LIFESTYLES PROMOTION TO CHILDREN AND FAMILIES: RESULTS } \\
\text { OF A COHORT STUDY. Nutr Hosp.2015;32:2584-7. }\end{array}$ & Age \\
\hline 223 & $\begin{array}{l}\text { Gomez-Sanchiz, M.,Canete, R.,Rodero, I.,Baeza, J. E.,Avila, O. Influence of breast-feeding on mental and psychomotor } \\
\text { development. Clin Pediatr (Phila).2003;42:35-42. }\end{array}$ & IV \\
\hline 224 & $\begin{array}{l}\text { Gomez-Sanchiz, M.,Canete, R.,Rodero, I.,Baeza, J. E.,Gonzalez, J. A. Influence of breast-feeding and parental intelligence on } \\
\text { cognitive development in the 24-month-old child. Clin Pediatr (Phila).2004;43:753-61. }\end{array}$ & IV \\
\hline 225 & $\begin{array}{l}\text { Gong, Y. H.,Ji, C. Y.,Zheng, X. X.,Shan, J. P.,Hou, R. Correlation of 4-month infant feeding modes with their growth and iron } \\
\text { status in Beijing. Chin Med J (Engl).2008;121:392-8. }\end{array}$ & IV \\
\hline 226 & $\begin{array}{l}\text { Gooze, R. A.,Anderson, S. E.,Whitaker, R. C. Prolonged bottle use and obesity at } 5.5 \text { years of age in US children. J } \\
\text { Pediatr.2011;159:431-6. }\end{array}$ & IV \\
\hline 227 & $\begin{array}{l}\text { Greene, L. C.,Lucas, A.,Livingstone, M. B.,Harland, P. S.,Baker, B. A. Relationship between early diet and subsequent cognitive } \\
\text { performance during adolescence. Biochem Soc Trans.1995;23:376s. }\end{array}$ & Design, IV \\
\hline 228 & $\begin{array}{l}\text { Greer,F. R.,Searcy,J. E.,Levin,R. S.,Steichen,J. J.,Steichen-Asche,P. S.,Tsang,R. C. Bone mineral content and serum 25- } \\
\text { hydroxyvitamin D concentrations in breast-fed infants with and without supplemental vitamin D: one-year follow-up. J } \\
\text { Pediatr.1982;100:919-22. }\end{array}$ & IV \\
\hline 229 & $\begin{array}{l}\text { Grote, V.,von Kries, R.,Closa-Monasterolo, R.,Scaglioni, S.,Gruszfeld, D.,Sengier, A.,Langhendries, J. P.,Koletzko, B. Protein } \\
\text { intake and growth in the first } 24 \text { months of life. J Pediatr Gastroenterol Nutr.2010;51 Suppl 3:S117-8. }\end{array}$ & Design, IV \\
\hline 230 & $\begin{array}{l}\text { Gruszfeld, D.,Weber, M.,Nowakowska-Rysz, M.,Janas, R.,Kozlik-Feldmann, R., Xhonneux, A.,Carlier, C.,Riva, E.,Verduci, } \\
\text { E.,Closa-Monasterolo, R.,Escribano, J.,Dobrzanska, A.,Koletzko, B. Protein intake in infancy and carotid intima media thickness } \\
\text { at } 5 \text { years--a secondary analysis from a randomized trial. Ann Nutr Metab.2015;66:51-9. }\end{array}$ & IV \\
\hline 231 & $\begin{array}{l}\text { Gubbels, J. S.,Thijs, C.,Stafleu, A.,van Buuren, S.,Kremers, S. P. Association of breast-feeding and feeding on demand with child } \\
\text { weight status up to } 4 \text { years. Int J Pediatr Obes.2011;6:e515-22. }\end{array}$ & IV, DV \\
\hline 232 & $\begin{array}{l}\text { Gunderson, E. P.,Hurston, S. R.,Dewey, K. G.,Faith, M. S.,Charvat-Aguilar, N.,Khoury, V. C.,Nguyen, V. T.,Quesenberry, C. P., } \\
\text { Jr. The study of women, infant feeding and type } 2 \text { diabetes after GDM pregnancy and growth of their offspring (SWIFT Offspring } \\
\text { study): prospective design, methodology and baseline characteristics. BMC Pregnancy Childbirth.2015;15:150. }\end{array}$ & Design \\
\hline 233 & $\begin{array}{l}\text { Gunnarsdottir I,Schack-Nielsen L,Michaelsen KF,Sorensen TI,Thorsdottir I. Infant weight gain, duration of exclusive breast- } \\
\text { feeding and childhood BMI - two similar follow-up cohorts. Public Health Nutr.2010;13:201-7. }\end{array}$ & IV \\
\hline
\end{tabular}




\begin{tabular}{|c|c|c|}
\hline 234 & $\begin{array}{l}\text { Gunnarsdottir, I.,Thorsdottir, I. Relationship between growth and feeding in infancy and body mass index at the age of } 6 \text { years. } \\
\text { Int J Obes Relat Metab Disord.2003;27:1523-7. }\end{array}$ & IV \\
\hline 235 & $\begin{array}{l}\text { Gunther, A. L.,Buyken, A. E.,Kroke, A. Protein intake during the period of complementary feeding and early childhood and the } \\
\text { association with body mass index and percentage body fat at } 7 \text { y of age. Am J Clin Nutr.2007;85:1626-33. }\end{array}$ & IV \\
\hline 236 & $\begin{array}{l}\text { Gunther, A. L.,Buyken, A. E.,Kroke, A. The influence of habitual protein intake in early childhood on BMI and age at adiposity } \\
\text { rebound: results from the DONALD Study. Int J Obes (Lond).2006;30:1072-9. }\end{array}$ & IV \\
\hline 237 & $\begin{array}{l}\text { Gurnida, D. A., Rowan, A. M.,Idjradinata, P.,Muchtadi, D.,Sekarwana, N. Association of complex lipids containing gangliosides } \\
\text { with cognitive development of 6-month-old infants. Early Hum Dev.2012;88:595-601. }\end{array}$ & IV \\
\hline 238 & $\begin{array}{l}\text { Hamilton, K.,Daniels, L.,White, K. M.,Murray, N.,Walsh, A. Predicting mothers' decisions to introduce complementary feeding at } 6 \\
\text { months. An investigation using an extended theory of planned behaviour. Appetite.2011;56:674-81. }\end{array}$ & IV, DV \\
\hline 239 & $\begin{array}{l}\text { Hammer, L. D.,Bryson, S.,Agras, W. S. Development of feeding practices during the first } 5 \text { years of life. Arch Pediatr Adolesc } \\
\text { Med.1999;153:189-94. }\end{array}$ & DV \\
\hline 240 & $\begin{array}{l}\text { Han,Y. H.,Yon,M.,Han,H. S.,Johnston,K. E.,Tamura,T.,Hyun, T. Zinc status and growth of Korean infants fed human milk, casein- } \\
\text { based, or soy-based formula: three-year longitudinal study. Nutr Res Pract.2011;5:46-51. }\end{array}$ & IV \\
\hline 241 & $\begin{array}{l}\text { Harbild, H. L., Harslof, L. B.,Christensen, J. H.,Kannass, K. N.,Lauritzen, L. Fish oil-supplementation from } 9 \text { to } 12 \text { months of age } \\
\text { affects infant attention in a free-play test and is related to change in blood pressure. Prostaglandins Leukot Essent Fatty } \\
\text { Acids.2013;89:327-33. }\end{array}$ & IV \\
\hline 242 & $\begin{array}{l}\text { Harit D,Faridi MM,Aggarwal A,Sharma SB. Lipid profile of term infants on exclusive breastfeeding and mixed feeding: a } \\
\text { comparative study. Eur J Clin Nutr.2008;62:203-9. }\end{array}$ & IV \\
\hline 243 & $\begin{array}{l}\text { Harrington,M.,Hotz,C.,Zeder,C.,Polvo,G. O.,Villalpando,S.,Zimmermann,M. B.,Walczyk,T.,Rivera,J. A.,Hurrell,R. F. A comparison } \\
\text { of the bioavailability of ferrous fumarate and ferrous sulfate in non-anemic Mexican women and children consuming a sweetened } \\
\text { maize and milk drink. Eur J Clin Nutr.2011;65:20-5. }\end{array}$ & IV, DV \\
\hline 244 & Harris, R. Inadequate diets: short- and long-term problems. J Fam Health Care.2002;12:6. & Design \\
\hline 245 & $\begin{array}{l}\text { Hasan,J.,Ray,J.,Khan,Z. Role of weaning in the nutritional status of infant--a longitudinal study in the rural area of Aligarh. J } \\
\text { Indian Med Assoc.1996;94:169, } 215 \text {. }\end{array}$ & $\begin{array}{l}\text { Design, IV, } \\
\text { DV }\end{array}$ \\
\hline 246 & $\begin{array}{l}\text { Haschke, F.,Pietschnig, B.,Vanura, H.,Heil, M.,Steffan, I.,Hobiger, G.,Schuster, E.,Camaya, Z. Iron intake and iron nutritional } \\
\text { status of infants fed iron-fortified beikost with meat. Am J Clin Nutr.1988;47:108-12. }\end{array}$ & IV \\
\hline 247 & $\begin{array}{l}\text { Hawley, N. L.,Johnson, W.,Nu'usolia, O.,McGarvey, S. T. The contribution of feeding mode to obesogenic growth trajectories in } \\
\text { American Samoan infants. Pediatr Obes.2014;9:e1-e13. }\end{array}$ & IV \\
\hline 248 & $\begin{array}{l}\text { Hay,G.,Johnston,C.,Whitelaw,A.,Trygg,K.,Refsum,H. Folate and cobalamin status in relation to breastfeeding and weaning in } \\
\text { healthy infants. Am J Clin Nutr.2008;88:105-14. }\end{array}$ & DV \\
\hline 249 & $\begin{array}{l}\text { Hediger, M. L.,Overpeck, M. D.,Ruan, W. J.,Troendle, J. F. Early infant feeding and growth status of US-born infants and children } \\
\text { aged 4-71 mo: analyses from the third National Health and Nutrition Examination Survey, 1988-1994. Am J Clin } \\
\text { Nutr.2000;72:159-67. }\end{array}$ & Design, IV \\
\hline
\end{tabular}




\begin{tabular}{|c|c|c|}
\hline 250 & $\begin{array}{l}\text { Hegar, B.,Dewanti, N. R.,Kadim, M.,Alatas, S.,Firmansyah, A.,Vandenplas, Y. Natural evolution of regurgitation in healthy infants. } \\
\text { Acta Paediatr.2009;98:1189-93. }\end{array}$ & IV, DV \\
\hline 251 & $\begin{array}{l}\text { Heine, W.,Lapsien, C. Influence of early breast milk and formula feeding on body weight in children born in Rostock since } 1945 . \\
\text { Bibl Nutr Dieta.1982:17-8. }\end{array}$ & Design \\
\hline 252 & $\begin{array}{l}\text { Herbst, A.,Diethelm, K.,Cheng, G.,Alexy, U.,Icks, A.,Buyken, A. E. Direction of associations between added sugar intake in early } \\
\text { childhood and body mass index at age } 7 \text { years may depend on intake levels. J Nutr.2011;141:1348-54. }\end{array}$ & IV \\
\hline 253 & $\begin{array}{l}\text { Hess,S. Y.,Abbeddou,S.,Jimenez,E. Y.,Somé,J. W.,Vosti,S. A.,Ouédraogo,Z. P.,Guissou,R. M.,Ouédraogo,J. B.,Brown,K. H. } \\
\text { Small-quantity lipid-based nutrient supplements, regardless of their zinc content, increase growth and reduce the prevalence of } \\
\text { stunting and Wasting in young burkinabe children: A cluster-randomized trial. PLoS ONE.2015;10. }\end{array}$ & $\begin{array}{l}\text { IV, } \\
\text { Country }\end{array}$ \\
\hline 254 & $\begin{array}{l}\text { Hettiarachchi,M.,Lekamwasam,S.,Liyanage,C. Long-term cereal-based nutritional supplementation improved the total spine bone } \\
\text { mineral density amongst Sri Lankan preschool children: a randomized controlled study. J Pediatr Endocrinol Metab.2010;23:555- } \\
63 .\end{array}$ & IV, Age \\
\hline 255 & $\begin{array}{l}\text { Hijazi, S. S.,Abulaban, A.,Waterlow, J. C. The duration for which exclusive breast-feeding is adequate. A study in Jordan. Acta } \\
\text { Paediatr Scand.1989;78:23-8. }\end{array}$ & IV \\
\hline 256 & $\begin{array}{l}\text { Hillis, S. D., Weigle, K.,Miranda, C. M.,McCann, M.,Bender, D.,Stewart, P. Supplementary feeding in Colombian child-care centers } \\
\text { and growth velocity. Pediatrics.1994;94:1066-9. }\end{array}$ & IV \\
\hline 257 & $\begin{array}{l}\text { Hitchcock, N. E.,Gracey, M.,Gilmour, A. I. The growth of breast fed and artificially fed infants from birth to twelve months. Acta } \\
\text { Paediatr Scand.1985;74:240-5. }\end{array}$ & IV \\
\hline 258 & $\begin{array}{l}\text { Hitchcock, N. E.,McGuiness, D.,Gracey, M. Growth and feeding practices of Western Australian infants. Med J Aust.1982;1:372- } \\
6 .\end{array}$ & $\begin{array}{l}\text { Design, IV, } \\
\text { DV, Date }\end{array}$ \\
\hline 259 & Hitchcock, N. E.,Owles, E. N.,Gracey, M. Breast feeding and growth of healthy infants. Med J Aust.1981;2:536-7. & $\begin{array}{l}\text { IV, DV, } \\
\text { Date }\end{array}$ \\
\hline 260 & $\begin{array}{l}\text { Hoffman, D. R.,Garfield, S.,Morale, S. E.,Bosworth, R. G.,Castaneda, Y. S., Wheaton, D. K. H.,Theuer, R. C.,Birch, E. E. Visual } \\
\text { and neural development of breast-fed infants receiving docosahexaenoic acid (dha)-enriched baby food: a randomized clinical } \\
\text { trial. lovs.2004;45:ARVO E-abstract } 3510 .\end{array}$ & Design \\
\hline 261 & $\begin{array}{l}\text { Hokama,T. Levels of serum ferritin and total body iron among infants with different feeding regimens. Acta Paediatr } \\
\text { Jpn.1993;35:298-301. }\end{array}$ & IV, DV \\
\hline 262 & $\begin{array}{l}\text { Holgerson PL,Vestman NR,Claesson R,Ohman C,Domellof M,Tanner AC,Hernell O,Johansson I. Oral microbial profile } \\
\text { discriminates breast-fed from formula-fed infants. J Pediatr Gastroenterol Nutr.2013;56:127-36. }\end{array}$ & $\begin{array}{l}\text { Design, IV, } \\
\text { DV }\end{array}$ \\
\hline 263 & $\begin{array}{l}\text { Hop le, T.,Berger, J. Multiple micronutrient supplementation improves anemia, micronutrient nutrient status, and growth of } \\
\text { Vietnamese infants: double-blind, randomized, placebo-controlled trial. J Nutr.2005;135:660s-665s. }\end{array}$ & IV \\
\hline 264 & $\begin{array}{l}\text { Hop, L. T.,Gross, R.,Giay, T.,Sastroamidjojo, S.,Schultink, W.,Lang, N. T. Premature complementary feeding is associated with } \\
\text { poorer growth of vietnamese children. J Nutr.2000;130:2683-90. }\end{array}$ & Country \\
\hline 265 & Hop, L. T.,Gross, R.,Giay, T.,Schultink, W.,Thuan, B. T.,Sastroamidjojo, S. Longitudinal observation of growth of Vietnamese & IV, DV \\
\hline
\end{tabular}




\begin{tabular}{|c|c|c|}
\hline & children in Hanoi, Vietnam from birth to 10 years of age. Eur J Clin Nutr.1997;51:164-71. & \\
\hline 266 & $\begin{array}{l}\text { Hopkins, D.,Steer, C. D.,Northstone, K.,Emmett, P. M. Effects on childhood body habitus of feeding large volumes of cow or } \\
\text { formula milk compared with breastfeeding in the latter part of infancy. Am J Clin Nutr.2015;102:1096-103. }\end{array}$ & IV \\
\hline 267 & $\begin{array}{l}\text { Hopkins,D.,Emmett,P.,Steer,C.,Rogers,I.,Noble,S.,Emond,A. Infant feeding in the second } 6 \text { months of life related to iron status: } \\
\text { an observational study. Arch Dis Child.2007;92:850-4. }\end{array}$ & DV \\
\hline 268 & $\begin{array}{l}\text { Hoppe, C.,Molgaard, C.,Thomsen, B. L.,Juul, A.,Michaelsen, K. F. Protein intake at } 9 \text { mo of age is associated with body size but } \\
\text { not with body fat in 10-y-old Danish children. Am J Clin Nutr.2004;79:494-501. }\end{array}$ & IV \\
\hline 269 & $\begin{array}{l}\text { Horodynski, M. A.,Baker, S.,Van Egeren, L.,Olson, B.,Brophy-Herb, H.,Auld, G. The healthy babies curriculum. J Nutr Educ } \\
\text { Behav.2014;46:151-2. }\end{array}$ & Design, IV \\
\hline 270 & $\begin{array}{l}\text { Horta, B. L.,Bas, A.,Bhargava, S. K.,Fall, C. H.,Feranil, A.,de Kadt, J.,Martorell, R.,Richter, L. M.,Stein, A. D.,Victora, C. G. Infant } \\
\text { feeding and school attainment in five cohorts from low- and middle-income countries. PLoS One.2013;8:e71548. }\end{array}$ & Design \\
\hline 271 & Horwood, L. J.,Fergusson, D. M. Breastfeeding and later cognitive and academic outcomes. Pediatrics.1998;101:E9. & IV \\
\hline 272 & $\begin{array}{l}\text { Hosaka, M.,Asayama, K.,Staessen, J. A.,Ohkubo, T.,Hayashi, K.,Tatsuta, N.,Kurokawa, N.,Satoh, M.,Hashimoto, T.,Hirose, } \\
\text { T.,Obara, T.,Metoki, H.,Inoue, R.,Kikuya, M.,Nakai, K.,Imai, Y.,Satoh, H. Breastfeeding leads to lower blood pressure in 7-year- } \\
\text { old Japanese children: Tohoku Study of Child Development. Hypertens Res.2013;36:117-22. }\end{array}$ & IV, DV \\
\hline 273 & $\begin{array}{l}\text { Hudara S,Mimouni F,Rachman Y,Dayan B,Silbermintz A,Turner D. Retrospective cohort study of a new infant formula during the } \\
\text { first } 6 \text { months of life: reflections on growth curves, human milk and formula feeding. Isr Med Assoc J.2010;12:676-80. }\end{array}$ & IV \\
\hline 274 & $\begin{array}{l}\text { Hurley, K. M.,Black, M. M. Introduction to a supplement on responsive feeding: promoting healthy growth and development for } \\
\text { infants and toddlers. J Nutr.2011;141:489. }\end{array}$ & Design \\
\hline 275 & $\begin{array}{l}\text { Husaini, M. A.,Karyadi, L.,Husaini, Y. K.,Sandjaja,,Karyadi, D.,Pollitt, E. Developmental effects of short-term supplementary } \\
\text { feeding in nutritionally-at-risk Indonesian infants. Am J Clin Nutr.1991;54:799-804. }\end{array}$ & Country \\
\hline 276 & $\begin{array}{l}\text { Inostroza, J.,Haschke, F.,Steenhout, P.,Grathwohl, D.,Nelson, S. E.,Ziegler, E. E. Low-protein formula slows weight gain in } \\
\text { infants of overweight mothers. J Pediatr Gastroenterol Nutr.2014;59:70-7. }\end{array}$ & IV \\
\hline 277 & $\begin{array}{l}\text { Isherwood,R. J.,Dimond,C.,Longhurst,S. Breast feeding and weaning practices in relation to nutritional status of under-5 children } \\
\text { in north Bangladesh. J Trop Pediatr.1988;34:28-31. }\end{array}$ & Design \\
\hline 278 & $\begin{array}{l}\text { Islam, M. M.,Peerson, J. M.,Ahmed, T.,Dewey, K. G.,Brown, K. H. Effects of varied energy density of complementary foods on } \\
\text { breast-milk intakes and total energy consumption by healthy, breastfed Bangladeshi children. Am J Clin Nutr.2006;83:851-8. }\end{array}$ & IV, DV \\
\hline 279 & $\begin{array}{l}\text { Jackson, D. A.,Imong, S. M.,Wongsawasdii, L.,Silprasert, A.,Preunglampoo, S.,Leelapat, P.,Drewett, R. F.,Amatayakul, K.,Baum, } \\
\text { J. D. Weaning practices and breast-feeding duration in Northern Thailand. Br J Nutr.1992;67:149-64. }\end{array}$ & IV, DV \\
\hline 280 & $\begin{array}{l}\text { Jackson,M. I. Early childhood WIC participation, cognitive development and academic achievement. Soc Sci Med.2015;126:145- } \\
53 .\end{array}$ & IV \\
\hline 281 & $\begin{array}{l}\text { Jacobson, S. W.,Chiodo, L. M.,Jacobson, J. L. Breastfeeding effects on intelligence quotient in 4- and 11-year-old children. } \\
\text { Pediatrics.1999;103:e71. }\end{array}$ & IV \\
\hline
\end{tabular}




\begin{tabular}{|c|c|c|}
\hline 282 & $\begin{array}{l}\text { Jain,V.,Klein,B. P.,Nash,M.,Chapman-Novakofski,K. Two feasibility studies for introduction of multimicronutrient soy/whey-based } \\
\text { supplements in rural homes in Honduras. Journal of Hunger and Environmental Nutrition.2011;6:247-263. }\end{array}$ & IV \\
\hline 283 & Jansen,G.R.,O'B Hourihane. The nutritional status of preschool children in Egypt. World Rev Nutr Diet.1985;45:42-67. & Design \\
\hline 284 & $\begin{array}{l}\text { Jatanasen,A.,Sacholvicharn,V.,Ongkulna,P. An evaluation of supplementary feedings of Thai infants in urban slum communities: } \\
\text { effect on growth, health and nutritional status. J Med Assoc Thai.1983;66 Suppl 1:20-9. }\end{array}$ & $\begin{array}{l}\text { Design, } \\
\text { Health } \\
\text { status }\end{array}$ \\
\hline 285 & $\begin{array}{l}\text { Jensen, B. W.,Nichols, M.,Allender, S.,de Silva-Sanigorski, A.,Millar, L.,Kremer, P.,Lacy, K.,Swinburn, B. Inconsistent } \\
\text { associations between sweet drink intake and 2-year change in BMl among Victorian children and adolescents. Pediatr } \\
\text { Obes.2013;8:271-83. }\end{array}$ & Age \\
\hline 286 & $\begin{array}{l}\text { Jensen, S. M.,Ritz, C.,Ejlerskov, K. T.,Molgaard, C.,Michaelsen, K. F. Infant BMI peak, breastfeeding, and body composition at } \\
\text { age } 3 \text { y. Am J Clin Nutr.2015;101:319-25. }\end{array}$ & IV \\
\hline 287 & $\begin{array}{l}\text { Jensen,C. L.,Prager,T. C.,Zou,Y.,Fraley,J. K.,Maude,M.,Anderson,R. E.,Heird,W. C. Effects of maternal docosahexaenoic acid } \\
\text { supplementation on visual function and growth of breast-fed term infants. Lipids.1999;34 Suppl:S225. }\end{array}$ & Design, IV \\
\hline 288 & $\begin{array}{l}\text { Jones, G.,Hynes, K. L.,Dwyer, T. The association between breastfeeding, maternal smoking in utero, and birth weight with bone } \\
\text { mass and fractures in adolescents: a 16-year longitudinal study. Osteoporos Int.2013;24:1605-11. }\end{array}$ & IV \\
\hline 289 & $\begin{array}{l}\text { Jones, G.,Riley, M.,Dwyer, T. Breastfeeding in early life and bone mass in prepubertal children: a longitudinal study. Osteoporos } \\
\text { Int.2000;11:146-52. }\end{array}$ & IV \\
\hline 290 & $\begin{array}{l}\text { Jooste, P. L., Rossouw, L. J.,Steenkamp, H. J.,Rossouw, J. E.,Swanepoel, A. S.,Charlton, D. O. Effect of breast feeding on the } \\
\text { plasma cholesterol and growth of infants. J Pediatr Gastroenterol Nutr.1991;13:139-42. }\end{array}$ & IV \\
\hline 291 & $\begin{array}{l}\text { Joseph, N.,Unnikrishnan, B.,Naik, V. A.,Mahantshetti, N. S.,Mallapur, M. D.,Kotian, S. M.,Nelliyanil, M. Infant rearing practices in } \\
\text { South India: a longitudinal study. J Family Med Prim Care.2013;2:37-43. }\end{array}$ & Country \\
\hline 292 & $\begin{array}{l}\text { Joshi N,Agho KE,Dibley MJ,Senarath U,Tiwari K. Determinants of inappropriate complementary feeding practices in young } \\
\text { children in Nepal: secondary data analysis of Demographic and Health Survey 2006. Matern Child Nutr.2012;8 Suppl 1:45-59. }\end{array}$ & $\begin{array}{l}\text { Design, } \\
\text { Country }\end{array}$ \\
\hline 293 & $\begin{array}{l}\text { Juez, G.,Diaz, S.,Casado, M. E.,Duran, E.,Salvatierra, A. M.,Peralta, O.,Croxatto, H. B. Growth pattern of selected urban Chilean } \\
\text { infants during exclusive breast-feeding. Am J Clin Nutr.1983;38:462-8. }\end{array}$ & IV \\
\hline 294 & $\begin{array}{l}\text { Jung, E.,Czajka-Narins, D. M. Birth weight doubling and tripling times: an updated look at the effects of birth weight, sex, race and } \\
\text { type of feeding. Am J Clin Nutr.1985;42:182-9. }\end{array}$ & IV \\
\hline 295 & $\begin{array}{l}\text { Kagura, J.,Feeley, A. B.,Micklesfield, L. K.,Pettifor, J. M.,Norris, S. A. Association between infant nutrition and anthropometry, } \\
\text { and pre-pubertal body composition in urban South African children. J Dev Orig Health Dis.2012;3:415-23. }\end{array}$ & Country \\
\hline 296 & $\begin{array}{l}\text { Kainonen E,Rautava S,Isolauri E. Immunological programming by breast milk creates an anti-inflammatory cytokine milieu in } \\
\text { breast-fed infants compared to formula-fed infants. Br J Nutr.2013;109:1962-70. }\end{array}$ & IV, DV \\
\hline 297 & $\begin{array}{l}\text { Kajantie, E.,Barker, D. J.,Osmond, C.,Forsen, T.,Eriksson, J. G. Growth before } 2 \text { years of age and serum lipids } 60 \text { years later: the } \\
\text { Helsinki Birth Cohort study. Int J Epidemiol.2008;37:280-9. }\end{array}$ & DV \\
\hline
\end{tabular}




\begin{tabular}{|c|c|c|}
\hline 298 & $\begin{array}{l}\text { Kallio, M. J.,Salmenpera, L.,Siimes, M. A.,Perheentupa, J.,Miettinen, T. A. Tracking of serum cholesterol and lipoprotein levels } \\
\text { from the first year of life. Pediatrics.1993;91:949-54. }\end{array}$ & IV, DV \\
\hline 299 & $\begin{array}{l}\text { Kallio,M. J.,Salmenpera,L.,Siimes,M. A.,Perheentupa,J.,Miettinen,T. A. Exclusive breast-feeding and weaning: effect on serum } \\
\text { cholesterol and lipoprotein concentrations in infants during the first year of life. Pediatrics.1992;89:663-6. }\end{array}$ & IV, DV \\
\hline 300 & $\begin{array}{l}\text { Karaolis-Danckert, N.,Buyken, A. E.,Kulig, M.,Kroke, A.,Forster, J.,Kamin, W.,Schuster, A.,Hornberg, C.,Keil, T.,Bergmann, R. } \\
\text { L.,Wahn, U.,Lau, S. How pre- and postnatal risk factors modify the effect of rapid weight gain in infancy and early childhood on } \\
\text { subsequent fat mass development: results from the Multicenter Allergy Study 90. Am J Clin Nutr.2008;87:1356-64. }\end{array}$ & IV \\
\hline 301 & $\begin{array}{l}\text { Karlsland Akeson, P. K.,Axelsson, I. E., Raiha, N. C., Warm, A.,Minoli, I.,Moro, G. Protein intake and metabolism in formula-fed } \\
\text { infants given Swedish or Italian weaning foods. Acta Paediatr.2000;89:158-64. }\end{array}$ & IV \\
\hline 302 & $\begin{array}{l}\text { Karlsson Videhult, F.,Ohlund, I.,Stenlund, H.,Hernell, O.,West, C. E. Probiotics during weaning: a follow-up study on effects on } \\
\text { body composition and metabolic markers at school age. Eur J Nutr.2015;54:355-63. }\end{array}$ & IV \\
\hline 303 & $\begin{array}{l}\text { Kattelmann,K. K.,Ho,M.,Specker,B. L. Effect of timing of introduction of complementary foods on iron and zinc status of formula } \\
\text { fed infants at 12, 24, and } 36 \text { months of age. J Am Diet Assoc.2001;101:443-7. }\end{array}$ & DV \\
\hline 304 & $\begin{array}{l}\text { Kavanagh, K. F.,Cohen, R. J.,Heinig, M. J.,Dewey, K. G. Educational intervention to modify bottle-feeding behaviors among } \\
\text { formula-feeding mothers in the WIC program: impact on infant formula intake and weight gain. J Nutr Educ Behav.2008;40:244- } \\
50 .\end{array}$ & IV \\
\hline 305 & $\begin{array}{l}\text { Kavle, J. A.,Flax, V. L.,Abdelmegeid, A.,Salah, F.,Hafez, S.,Ramzy, M.,Hamed, D.,Saleh, G.,Galloway, R. Factors associated } \\
\text { with early growth in Egyptian infants: implications for addressing the dual burden of malnutrition. Matern Child Nutr.2016;12:139- } \\
51 .\end{array}$ & Country \\
\hline 306 & $\begin{array}{l}\text { Kersting, M.,Alexy, U.,Sichert-Hellert, W.,Manz, F.,Schoch, G. Measured consumption of commercial infant food products in } \\
\text { German infants: results from the DONALD study. Dortmund Nutritional and Anthropometrical Longitudinally Designed. J Pediatr } \\
\text { Gastroenterol Nutr.1998;27:547-52. }\end{array}$ & DV \\
\hline 307 & $\begin{array}{l}\text { Kesaree, N.,Shivamurty, K. S.,Prakash, B. S., Ramachandra,,Haridas, C. K. Feeding pattern of infants in Devangere. Indian J } \\
\text { Pediatr.1981;48:281-4. }\end{array}$ & Design, IV \\
\hline 308 & Khaldi, F.,Bouguerra, F. Feeding practices, growth, and morbidity in Tunisia. Pediatrics.2000;106:1275-6. & $\begin{array}{l}\text { Design, } \\
\text { Date }\end{array}$ \\
\hline 309 & $\begin{array}{l}\text { Khan, A. I.,Kabir, I.,Hawkesworth, S.,Ekström, E. C.,Arifeen, S.,Frongillo, E. A.,Persson, L. A. Early invitation to food and/or } \\
\text { multiple micronutrient supplementation in pregnancy does not affect body composition in offspring at } 54 \text { months: Follow-up of the } \\
\text { MINIMat randomised trial, Bangladesh. Maternal and Child Nutrition.2015;11:385-397. }\end{array}$ & IV, Age \\
\hline 310 & Khan, M. U. Breastfeeding, growth and diarrhoea in rural Bangladesh children. Hum Nutr Clin Nutr.1984;38:113-9. & IV \\
\hline 311 & Khan, M. Infant feeding practices in rural Meheran, Comilla, Bangladesh. Am J Clin Nutr.1980;33:2356-64. & IV, DV \\
\hline 312 & $\begin{array}{l}\text { Kholdi N,Zayeri F,Bagheban AA,Khodakarim S,Ramezankhani A. A study of growth failure and its related factors in children from } \\
0 \text { to } 2 \text { years in Tehran, Iran. Turk J Pediatr.2012;54:38-44. }\end{array}$ & Design \\
\hline
\end{tabular}




\begin{tabular}{|c|c|c|}
\hline 313 & $\begin{array}{l}\text { Kiefte-de Jong, J. C., de Vries, J. H.,Escher, J. C.,Jaddoe, V. W., Hofman, A., Raat, H.,Moll, H. A. Role of dietary patterns, } \\
\text { sedentary behaviour and overweight on the longitudinal development of childhood constipation: the Generation R study. Matern } \\
\text { Child Nutr.2013;9:511-23. }\end{array}$ & DV \\
\hline 314 & $\begin{array}{l}\text { Kilaru, A.,Griffiths, P. L.,Ganapathy, S.,Ghosh, S. Community-based nutrition education for improving infant growth in rural } \\
\text { Karnataka. Indian Pediatr.2005;42:425-32. }\end{array}$ & IV \\
\hline 315 & $\begin{array}{l}\text { King,S.,Prawitz,A. D.,Umoren,J.,O'Gorman,T. The impact of high diastase malted barley flour on weight and height of } \\
\text { malnourished children in Panama. Journal of Hunger and Environmental Nutrition.2007;1:23-35. }\end{array}$ & $\begin{array}{l}\text { Health } \\
\text { status, } \\
\text { Age }\end{array}$ \\
\hline 316 & $\begin{array}{l}\text { Klag, E. A.,McNamara, K.,Geraghty, S. R.,Keim, S. A. Associations Between Breast Milk Feeding, Introduction of Solid Foods, } \\
\text { and Weight Gain in the First } 12 \text { Months of Life. Clin Pediatr (Phila).2015;54:1059-67. }\end{array}$ & Design \\
\hline 317 & Kleinman, R. E. Complementary feeding and neuromuscular development. Pediatrics.2000;106:1279. & Design \\
\hline 318 & $\begin{array}{l}\text { Kolacek, S.,Kapetanovic, T.,Zimolo, A.,Luzar, V. Early determinants of cardiovascular risk factors in adults. A. Plasma lipids. } \\
\text { Acta Paediatr.1993;82:699-704. }\end{array}$ & DV \\
\hline 319 & $\begin{array}{l}\text { Koletzko, B.,Dokoupil, K.,Reitmayr, S.,Weimert-Harendza, B.,Keller, E. Dietary fat intakes in infants and primary school children } \\
\text { in Germany. Am J Clin Nutr.2000;72:1392s-1398s. }\end{array}$ & IV, DV \\
\hline 320 & $\begin{array}{l}\text { Koletzko, B.,von Kries, R.,Closa, R.,Escribano, J.,Scaglioni, S.,Giovannini, M.,Beyer, J.,Demmelmair, H.,Anton, B.,Gruszfeld, } \\
\text { D.,Dobrzanska, A.,Sengier, A.,Langhendries, J. P.,Rolland Cachera, M. F.,Grote, V. Can infant feeding choices modulate later } \\
\text { obesity risk?. Am J Clin Nutr.2009;89:1502s-1508s. }\end{array}$ & Design, IV \\
\hline 321 & $\begin{array}{l}\text { Koletzko, B.,von Kries, R.,Closa, R.,Escribano, J.,Scaglioni, S.,Giovannini, M.,Beyer, J.,Demmelmair, H.,Gruszfeld, } \\
\text { D.,Dobrzanska, A.,Sengier, A.,Langhendries, J. P.,Rolland Cachera, M. F.,Grote, V. Lower protein in infant formula is associated } \\
\text { with lower weight up to age } 2 \text { y: a randomized clinical trial. Am J Clin Nutr.2009;89:1836-45. }\end{array}$ & IV \\
\hline 322 & Koletzko, B. Complementary foods and the development of food allergy. Pediatrics.2000;106:1285. & Design \\
\hline 323 & $\begin{array}{l}\text { Konstantynowicz, J.,Nguyen, T. V.,Kaczmarski, M.,Jamiolkowski, J.,Piotrowska-Jastrzebska, J.,Seeman, E. Fractures during } \\
\text { growth: potential role of a milk-free diet. Osteoporos Int.2007;18:1601-7. }\end{array}$ & Age \\
\hline 324 & $\begin{array}{l}\text { Kramer, M. S.,Fombonne, E.,Matush, L.,Bogdanovich, N.,Dahhou, M.,Platt, R. W. Long-term behavioural consequences of infant } \\
\text { feeding: the limits of observational studies. Paediatr Perinat Epidemiol.2011;25:500-6. }\end{array}$ & IV \\
\hline 325 & $\begin{array}{l}\text { Kramer, M. S.,Guo, T.,Platt, R. W.,Shapiro, S.,Collet, J. P.,Chalmers, B.,Hodnett, E.,Sevkovskaya, Z.,Dzikovich, I., Vanilovich, I. } \\
\text { Breastfeeding and infant growth: biology or bias?. Pediatrics.2002;110:343-7. }\end{array}$ & IV \\
\hline 326 & $\begin{array}{l}\text { Kramer, M. S.,Moodie, E. E.,Dahhou, M.,Platt, R. W. Breastfeeding and infant size: evidence of reverse causality. Am J } \\
\text { Epidemiol.2011;173:978-83. }\end{array}$ & IV, DV \\
\hline 327 & $\begin{array}{l}\text { Kramer, M. S.,Moodie, E. E.,Platt, R. W. Infant feeding and growth: can we answer the causal question?. } \\
\text { Epidemiology.2012;23:790-4. }\end{array}$ & Design \\
\hline 328 & Kramer, M. S. Do breast-feeding and delayed introduction of solid foods protect against subsequent obesity?. J & Design \\
\hline
\end{tabular}




\begin{tabular}{|c|c|c|}
\hline & Pediatr.1981;98:883-7. & \\
\hline 329 & $\begin{array}{l}\text { Krebs,N. F. Culbertson D. L. Westcott J. L. Sherlock L. Hambidge K. M. Normal Iron Status in Breastfed Infants Consuming Meat } \\
\text { as an Early Complementary Food. Pediatric Academic Societies Annual Meeting.2011. }\end{array}$ & Design \\
\hline 330 & $\begin{array}{l}\text { Krebs,N. F.,Hambidge,K. M.,Westcott,J. E.,Miller,L. V.,Sian,L.,Bell,M.,Grunwald,G. Exchangeable zinc pool size in infants is } \\
\text { related to key variables of zinc homeostasis. J Nutr.2003;133:1498s-501s. }\end{array}$ & DV \\
\hline 331 & $\begin{array}{l}\text { Krebs,N. F.,Mazariegos,M.,Chomba,E.,Sami,N.,Pasha,O.,Tshefu,A.,Carlo,W. A.,Goldenberg,R. L.,Bose,C. L.,Wright,L. L.,Koso- } \\
\text { Thomas,M.,Goco,N.,Kindem,M.,McClure,E. M.,Westcott,J.,Garces,A.,Lokangaka,A.,Manasyan,A.,Imenda,E.,Hartwell,T. } \\
\text { D.,Hambidge,K. M. Randomized controlled trial of meat compared with multimicronutrient-fortified cereal in infants and toddlers } \\
\text { with high stunting rates in diverse settings. Am J Clin Nutr.2012;96:840-7. }\end{array}$ & Country \\
\hline 332 & $\begin{array}{l}\text { Krebs,N. F.,Westcott,J. E.,Culbertson,D. L.,Sian,L.,Miller,L. V.,Hambidge,K. M. Comparison of complementary feeding strategies } \\
\text { to meet zinc requirements of older breastfed infants. Am J Clin Nutr.2012;96:30-5. }\end{array}$ & DV \\
\hline 333 & $\begin{array}{l}\text { Kronborg, H.,Foverskov, E.,Vaeth, M. Predictors for early introduction of solid food among Danish mothers and infants: an } \\
\text { observational study. BMC Pediatr.2014;14:243. }\end{array}$ & $\begin{array}{l}\text { Design, } \\
\text { DV }\end{array}$ \\
\hline 334 & $\begin{array}{l}\text { Kuklina, E. V.,Ramakrishnan, U.,Stein, A. D.,Barnhart, H. H.,Martorell, R. Growth and diet quality are associated with the } \\
\text { attainment of walking in rural Guatemalan infants. J Nutr.2004;134:3296-300. }\end{array}$ & IV \\
\hline 335 & $\begin{array}{l}\text { Kumar, V.,Sharma, S.,Khanna, P.,Vanaja, K. Breast vs bottle feeding-impact on growth in urban infants. Indian J } \\
\text { Pediatr.1981;48:271-5. }\end{array}$ & IV, DV \\
\hline 336 & $\begin{array}{l}\text { Kusin, J. A., Kardjati, S.,van Steenbergen, W. M.,Renqvist, U. H. Nutritional transition during infancy in East Java, Indonesia: } 2 \text {. A } \\
\text { longitudinal study of growth in relation to the intake of breast milk and additional foods. Eur J Clin Nutr.1991;45:77-84. }\end{array}$ & IV \\
\hline 337 & Kusin, J. A.,Kardjati, S.,van Steenbergen, W. Traditional infant feeding practices: right or wrong?. Soc Sci Med.1985;21:283-6. & $\begin{array}{l}\text { IV, } \\
\text { Country }\end{array}$ \\
\hline 338 & $\begin{array}{l}\text { Kwok, M. K.,Leung, G. M.,Lam, T. H.,Schooling, C. M. Breastfeeding, childhood milk consumption, and onset of puberty. } \\
\text { Pediatrics.2012;130:e631-9. }\end{array}$ & DV \\
\hline 339 & $\begin{array}{l}\text { Laitinen,K.,Kalliomaki,M.,Poussa,T.,Lagstrom,H.,Isolauri,E. Evaluation of diet and growth in children with and without atopic } \\
\text { eczema: follow-up study from birth to } 4 \text { years. Br J Nutr.2005;94:565-74. }\end{array}$ & IV, DV \\
\hline 340 & $\begin{array}{l}\text { Lampe,J. B.,Velez,N. The effect of prolonged bottle feeding on cow's milk intake and iron stores at } 18 \text { months of age. Clin } \\
\text { Pediatr (Phila).1997;36:569-72. }\end{array}$ & Design \\
\hline 341 & Lang B,Moynihan P. The weaning diet: promoting growth while safeguarding dental health. Quintessence Int.2006;37:632-6. & Design \\
\hline 342 & $\begin{array}{l}\text { Larnkjaer, A.,Hoppe, C.,Molgaard, C.,Michaelsen, K. F. The effects of whole milk and infant formula on growth and IGF-I in late } \\
\text { infancy. Eur J Clin Nutr.2009;63:956-63. }\end{array}$ & IV \\
\hline 343 & $\begin{array}{l}\text { Lasky, P. A.,Eichelberger, K. M. Implications, considerations, and nursing interventions of obesity in neonatal and preschool } \\
\text { patients. Nurs Clin North Am.1982;17:199-205. }\end{array}$ & Design \\
\hline
\end{tabular}




\begin{tabular}{|c|c|c|}
\hline 344 & $\begin{array}{l}\text { Lauzon-Guillain, Bd,Wijndaele, K.,Clark, M.,Acerini, C. L.,Hughes, I. A.,Dunger, D. B.,Wells, J. C.,Ong, K. K. Breastfeeding and } \\
\text { infant temperament at age three months. PLoS One.2012;7:e29326. }\end{array}$ & Design, IV \\
\hline 345 & $\begin{array}{l}\text { Lawlor, D. A.,Riddoch, C. J.,Page, A. S.,Andersen, L. B.,Wedderkopp, N.,Harro, M.,Stansbie, D.,Smith, G. D. Infant feeding and } \\
\text { components of the metabolic syndrome: findings from the European Youth Heart Study. Arch Dis Child.2005;90:582-8. }\end{array}$ & IV \\
\hline 346 & Lee, K. Crying and behavior pattern in breast- and formula-fed infants. Early Hum Dev.2000;58:133-40. & $\begin{array}{l}\text { Design, IV, } \\
\text { DV }\end{array}$ \\
\hline 347 & $\begin{array}{l}\text { Leermakers, E. T.,Kiefte-de Jong, J. C.,Hofman, A.,Jaddoe, V. W.,Franco, O. H. Lutein intake at the age of } 1 \text { year and } \\
\text { cardiometabolic health at the age of } 6 \text { years: the Generation R Study. Br J Nutr.2015;114:970-8. }\end{array}$ & IV \\
\hline 348 & Legovic, M.,Ostric, L. The effects of feeding methods on the growth of the jaws in infants. ASDC J Dent Child.1991;58:253-5. & $\begin{array}{l}\text { Design, IV, } \\
\text { DV }\end{array}$ \\
\hline 349 & $\begin{array}{l}\text { Lei, D. L.,Chaves, S. P.,Paes, A. T.,Escuder, M. M.,Ribeiro, A. B.,Freire, R. D.,Lerner, B. R. Risk of linear growth retardation } \\
\text { during the first two years of life: a new approach. Eur J Clin Nutr.1999;53:456-60. }\end{array}$ & IV \\
\hline 350 & $\begin{array}{l}\text { Leonard, W. R.,Dewalt, K. M.,Stansbury, J. P.,McCaston, M. K. Influence of dietary quality on the growth of highland and coastal } \\
\text { Ecuadorian children. Am J Hum Biol.2000;12:825-837. }\end{array}$ & IV \\
\hline 351 & $\begin{array}{l}\text { Leroy, J. L.,Ruel, M.,Habicht, J. P.,Frongillo, E. A. Using height-for-age differences (HAD) instead of height-for-age z-scores } \\
\text { (HAZ) for the meaningful measurement of population-level catch-up in linear growth in children less than } 5 \text { years of age. BMC } \\
\text { Pediatr.2015;15:145. }\end{array}$ & IV \\
\hline 352 & $\begin{array}{l}\text { Leung, S. S.,Peng, C. X.,Xu, Y. Y.,Liu, K. M.,Quan, X. J.,Lui, S.,Davies, D. P. Comparative study of growth of Chinese infants: } \\
\text { Hong Kong versus Guangzhou. J Trop Pediatr.1994;40:166-71. }\end{array}$ & IV, DV \\
\hline 353 & Leung,S.,Davies,D. P. Infant feeding and growth of Chinese infants: birth to 2 years. Paediatr Perinat Epidemiol.1994;8:301-13. & IV, DV \\
\hline 354 & $\begin{array}{l}\text { Leventakou, V.,Roumeliotaki, T.,Koutra, K.,Vassilaki, M.,Mantzouranis, E.,Bitsios, P.,Kogevinas, M.,Chatzi, L. Breastfeeding } \\
\text { duration and cognitive, language and motor development at } 18 \text { months of age: Rhea mother-child cohort in Crete, Greece. J } \\
\text { Epidemiol Community Health.2015;69:232-9. }\end{array}$ & IV \\
\hline 355 & $\begin{array}{l}\text { Li, C., Kaur, H.,Choi, W. S.,Huang, T. T.,Lee, R. E.,Ahluwalia, J. S. Additive interactions of maternal prepregnancy BMI and } \\
\text { breast-feeding on childhood overweight. Obes Res.2005;13:362-71. }\end{array}$ & IV \\
\hline 356 & $\begin{array}{l}\text { Li, R.,Magadia, J.,Fein, S. B.,Grummer-Strawn, L. M. Risk of bottle-feeding for rapid weight gain during the first year of life. Arch } \\
\text { Pediatr Adolesc Med.2012;166:431-6. }\end{array}$ & IV \\
\hline 357 & $\begin{array}{l}\text { Lin, S. L.,Leung, G. M.,Lam, T. H.,Schooling, C. M. Timing of solid food introduction and obesity: Hong Kong's "children of 1997" } \\
\text { birth cohort. Pediatrics.2013;131:e1459-67. }\end{array}$ & Design \\
\hline 358 & $\begin{array}{l}\text { Lind,T.,Lonnerdal,B.,Persson,L. A.,Stenlund,H.,Tennefors,C.,Hernell,O. Effects of weaning cereals with different phytate contents } \\
\text { on hemoglobin, iron stores, and serum zinc: a randomized intervention in infants from } 6 \text { to } 12 \text { mo of age. Am J Clin } \\
\text { Nutr.2003;78:168-75. }\end{array}$ & DV \\
\hline 359 & Livingstone V. Failure to thrive while breastfeeding. Breastfeed Med.2006;1:108-11. & Design \\
\hline
\end{tabular}




\begin{tabular}{|c|c|c|}
\hline 360 & $\begin{array}{l}\text { Lozoff, B.,De Andraca, I.,Castillo, M.,Smith, J. B.,Walter, T.,Pino, P. Behavioral and developmental effects of preventing iron- } \\
\text { deficiency anemia in healthy full-term infants. Pediatrics.2003;112:846-54. }\end{array}$ & IV \\
\hline 361 & $\begin{array}{l}\text { Lucas, R.,Judge, M.,Sajdlowska, J.,Cong, X.,McGrath, J. M.,Brandon, D. Effect of Maternal Body Mass Index on Infant } \\
\text { Breastfeeding Behaviors and Exclusive Direct Breastfeeding. J Obstet Gynecol Neonatal Nurs.2015;44:772-83. }\end{array}$ & DV \\
\hline 362 & $\begin{array}{l}\text { Lutter, C. K.,Mora, J. O.,Habicht, J. P.,Rasmussen, K. M.,Robson, D. S.,Herrera, M. G. Age-specific responsiveness of weight } \\
\text { and length to nutritional supplementation. Am J Clin Nutr.1990;51:359-64. }\end{array}$ & IV \\
\hline 363 & $\begin{array}{l}\text { Lutter, C. K.,Mora, J. O.,Habicht, J. P.,Rasmussen, K. M.,Robson, D. S.,Sellers, S. G.,Super, C. M.,Herrera, M. G. Nutritional } \\
\text { supplementation: effects on child stunting because of diarrhea. Am J Clin Nutr.1989;50:1-8. }\end{array}$ & IV \\
\hline 364 & $\begin{array}{l}\text { Lutter,C. K.,Rodriguez,A.,Fuenmayor,G.,Avila,L.,Sempertegui,F.,Escobar,J. Growth and micronutrient status in children receiving } \\
\text { a fortified complementary food. J Nutr.2008;138:379-88. }\end{array}$ & IV \\
\hline 365 & $\begin{array}{l}\text { Luukkainen,P.,Salo,M. K.,Visakorpi,J. K.,Raiha,N. C.,Nikkari,T. Impact of solid food on plasma arachidonic and docosahexaenoic } \\
\text { acid status of term infants at } 8 \text { months of age. J Pediatr Gastroenterol Nutr.1996;23:229-34. }\end{array}$ & IV, DV \\
\hline 366 & MacCarthy, D. Psychological influences affecting growth. Health Visit.1980;53:470-6. & Design \\
\hline 367 & $\begin{array}{l}\text { Magarey, A.,Kavian, F.,Scott, J. A.,Markow, K.,Daniels, L. Feeding Mode of Australian Infants in the First } 12 \text { Months of Life: An } \\
\text { Assessment against National Breastfeeding Indicators. J Hum Lact.2015. }\end{array}$ & DV \\
\hline 368 & $\begin{array}{l}\text { Maimaitiming, T.,Wang, Y. P. Feeding and intellectual development in infants and children from Uygur, Kazak, and Han } \\
\text { nationality: A comparison of } 454 \text { cases. Journal of Clinical Rehabilitative Tissue Engineering Research.2007;11:10710-10712. }\end{array}$ & Design \\
\hline 369 & $\begin{array}{l}\text { Makrides,M.,Gould,J. F.,Gawlik,N. R.,Yelland,L. N.,Smithers,L. G.,Anderson,P. J.,Gibson,R. A. Four-year follow-up of children } \\
\text { born to women in a randomized trial of prenatal DHA supplementation. Jama.2014;311:1802-4. }\end{array}$ & IV \\
\hline 370 & $\begin{array}{l}\text { Male,C.,Persson,L. A.,Freeman,V.,Guerra,A.,van't Hof,M. A.,Haschke,F. Prevalence of iron deficiency in 12-mo-old infants from } \\
11 \text { European areas and influence of dietary factors on iron status (Euro-Growth study). Acta Paediatr.2001;90:492-8. }\end{array}$ & DV \\
\hline 371 & $\begin{array}{l}\text { Mallan, K. M.,Fildes, A.,Magarey, A. M.,Daniels, L. A. The Relationship between Number of Fruits, Vegetables, and Noncore } \\
\text { Foods Tried at Age } 14 \text { Months and Food Preferences, Dietary Intake Patterns, Fussy Eating Behavior, and Weight Status at Age } \\
\text { 3.7 Years. J Acad Nutr Diet.2016;116:630-7. }\end{array}$ & DV \\
\hline 372 & $\begin{array}{l}\text { Mallard,S. R.,Houghton,L. A.,Filteau,S.,Mullen,A.,Nieuwelink,J.,Chisenga,M.,Siame,J.,Gibson,R. S. Dietary diversity at } 6 \text { months } \\
\text { of age is associated with subsequent growth and mediates the effect of maternal education on infant growth in urban Zambia. J } \\
\text { Nutr.2014;144:1818-25. }\end{array}$ & Country \\
\hline 373 & $\begin{array}{l}\text { Mamabolo, R. L.,Alberts, M.,Mbenyane, G. X.,Steyn, N. P.,Nthangeni, N. G.,Delemarre-Van De Waal, H. A.,Levitt, N. S. Feeding } \\
\text { practices and growth of infants from birth to } 12 \text { months in the central region of the Limpopo Province of South Africa. } \\
\text { Nutrition.2004;20:327-33. }\end{array}$ & IV, DV \\
\hline 374 & $\begin{array}{l}\text { Mamabolo,R. L.,Alberts,M.,Levitt,N. S.,Delemarre-van de Waal,H. A.,Steyn,N. P. Association between insulin-like growth factor-1, } \\
\text { insulin-like growth factor-binding protein-1 and leptin levels with nutritional status in 1-3-year-old children, residing in the central } \\
\text { region of Limpopo Province, South Africa. Br J Nutr.2007;98:762-9. }\end{array}$ & IV \\
\hline
\end{tabular}




\begin{tabular}{|c|c|c|}
\hline 375 & $\begin{array}{l}\text { Mamun, A. A.,Lawlor, D. A.,O'Callaghan, M. J.,Williams, G. M.,Najman, J. M. Family and early life factors associated with } \\
\text { changes in overweight status between ages } 5 \text { and } 14 \text { years: findings from the Mater University Study of Pregnancy and its } \\
\text { outcomes. Int J Obes (Lond).2005;29:475-82. }\end{array}$ & IV \\
\hline 376 & $\begin{array}{l}\text { Manjrekar, C.,Vishalakshi, M. P.,Begum, N. J.,Padma, G. N. Breast feeding ability of undernourished mothers and physical } \\
\text { development of their infants during 0-1 year. Indian Pediatr.1985;22:801-9. }\end{array}$ & IV, DV \\
\hline 377 & $\begin{array}{l}\text { Marques,R. C.,Dorea,J. G.,Bernardi,J. V.,Bastos,W. R.,Malm,O. Maternal fish consumption in the nutrition transition of the } \\
\text { Amazon Basin: growth of exclusively breastfed infants during the first } 5 \text { years. Ann Hum Biol.2008;35:363-77. }\end{array}$ & IV \\
\hline 378 & $\begin{array}{l}\text { Marquis, G. S.,Habicht, J. P.,Lanata, C. F.,Black, R. E., Rasmussen, K. M. Association of breastfeeding and stunting in Peruvian } \\
\text { toddlers: an example of reverse causality. Int J Epidemiol.1997;26:349-56. }\end{array}$ & Design \\
\hline 379 & $\begin{array}{l}\text { Marquis, G. S.,Habicht, J. P.,Lanata, C. F.,Black, R. E.,Rasmussen, K. M. Breast milk or animal-product foods improve linear } \\
\text { growth of Peruvian toddlers consuming marginal diets. Am J Clin Nutr.1997;66:1102-9. }\end{array}$ & Design \\
\hline 380 & $\begin{array}{l}\text { Martin, R. M.,McCarthy, A.,Smith, G. D.,Davies, D. P.,Ben-Shlomo, Y. Infant nutrition and blood pressure in early adulthood: the } \\
\text { Barry Caerphilly Growth study. Am J Clin Nutr.2003;77:1489-97. }\end{array}$ & IV, DV \\
\hline 381 & $\begin{array}{l}\text { Martin, R. M.,Smith, G. D.,Mangtani, P.,Frankel, S.,Gunnell, D. Association between breast feeding and growth: the Boyd-Orr } \\
\text { cohort study. Arch Dis Child Fetal Neonatal Ed.2002;87:F193-201. }\end{array}$ & IV \\
\hline 382 & $\begin{array}{l}\text { Martines, J. C.,Habicht, J. P.,Ashworth, A.,Kirkwood, B. R. Weaning in southern Brazil: is there a "weanling's dilemma"?. J } \\
\text { Nutr.1994;124:1189-98. }\end{array}$ & IV \\
\hline 383 & $\begin{array}{l}\text { Matsuzaki,M.,Kuper,H.,Kulkarni,B.,Radhakrishna,K. V.,Viljakainen,H.,Taylor,A. E.,Sullivan,R.,Bowen,L., Tobias,J. H.,Ploubidis,G. } \\
\text { B.,Wells,J. C.,Prabhakaran,D.,Smith,G. D.,Ebrahim,S.,Ben-Shlomo,Y.,Kinra,S. Life-course determinants of bone mass in young } \\
\text { adults from a transitional rural community in India: The Andhra Pradesh Children and Parents Study (APCAPS). American } \\
\text { Journal of Clinical Nutrition.2014;99:1450-1459. }\end{array}$ & $\begin{array}{l}\text { Design, IV, } \\
\text { Age }\end{array}$ \\
\hline 384 & $\begin{array}{l}\text { May, A. L.,Dietz, W. H. The Feeding Infants and Toddlers Study 2008: opportunities to assess parental, cultural, and } \\
\text { environmental influences on dietary behaviors and obesity prevention among young children. J Am Diet Assoc.2010;110:S11-5. }\end{array}$ & Design \\
\hline 385 & $\begin{array}{l}\text { Mazariegos,M.,Hambidge,K. M.,Westcott,J. E.,Solomons,N. W.,Raboy,V.,Das,A.,Goco,N.,Kindem,M.,Wright,L. L.,Krebs,N. F. } \\
\text { Neither a zinc supplement nor phytate-reduced maize nor their combination enhance growth of 6- to 12-month-old Guatemalan } \\
\text { infants. J Nutr.2010;140:1041-8. }\end{array}$ & Country \\
\hline 386 & McGuire, E. Breastfeeding and high maternal body mass index. Breastfeed Rev.2013;21:7-14. & Design \\
\hline 387 & $\begin{array}{l}\text { McNeill, G. Birth weight, feeding practices and weight-for-age of Punjabi children in the UK and in the rural Punjab. Hum Nutr } \\
\text { Clin Nutr.1985;39:69-72. }\end{array}$ & Design \\
\hline 388 & $\begin{array}{l}\text { Mehta KC,Specker BL,Bartholmey S,Giddens J,Ho ML. Trial on timing of introduction to solids and food type on infant growth. } \\
\text { Pediatrics.1998;102:569-73. }\end{array}$ & IV \\
\hline 389 & $\begin{array}{l}\text { Mei, H.,Guo, B.,Yin, B.,Liang, X.,Adair, L., Thompson, A.,Zhang, J. Interactive Effects of Early Exclusive Breastfeeding and Pre- } \\
\text { Pregnancy Maternal Weight Status on Young Children's BMI - A Chinese Birth Cohort. PLoS One.2015;10:e0144357. }\end{array}$ & IV \\
\hline
\end{tabular}




\begin{tabular}{|c|c|c|}
\hline 390 & $\begin{array}{l}\text { Meldrum,S. J.,D'Vaz,N.,Simmer,K.,Dunstan,J. A.,Hird,K.,Prescott,S. L. Effects of high-dose fish oil supplementation during early } \\
\text { infancy on neurodevelopment and language: a randomised controlled trial. Br J Nutr.2012;108:1443-54. }\end{array}$ & IV \\
\hline 391 & $\begin{array}{l}\text { Melnik, B. Growth and puberty in German children: is there still a positive secular trend? Consumption of milk as a vital factor in } \\
\text { growth development..Bettina Gohlke, Woelfle JF. volume 23/2009. Deutsches Aerzteblatt International.2009;106:656-656. }\end{array}$ & Design \\
\hline 392 & $\begin{array}{l}\text { Mennella, J. A., Castor, S. M. Sensitive period in flavor learning: effects of duration of exposure to formula flavors on food likes } \\
\text { during infancy. Clin Nutr.2012;31:1022-5. }\end{array}$ & DV \\
\hline 393 & $\begin{array}{l}\text { Merten, S.,Dratva, J.,Ackermann-Liebrich, U. Do baby-friendly hospitals influence breastfeeding duration on a national level?. } \\
\text { Pediatrics.2005;116:e702-8. }\end{array}$ & Design, IV \\
\hline 394 & $\begin{array}{l}\text { Michaelsen, K. F.,Johansen, J. S.,Samuelson, G.,Price, P. A., Christiansen, C. Serum bone gamma-carboxyglutamic acid protein } \\
\text { in a longitudinal study of infants: lower values in formula-fed infants. Pediatr Res.1992;31:401-5. }\end{array}$ & IV \\
\hline 395 & Michaelsen, K. F. Are there negative effects of an excessive protein intake?. Pediatrics.2000;106:1293. & Design \\
\hline 396 & $\begin{array}{l}\text { Michaelsen,K. F.,Milman,N.,Samuelson,G. A longitudinal study of iron status in healthy Danish infants: effects of early iron status, } \\
\text { growth velocity and dietary factors. Acta Paediatr.1995;84:1035-44. }\end{array}$ & IV, DV \\
\hline 397 & Michaelsen,K. F. Cows' milk in complementary feeding. Pediatrics.2000;106:1302-3. & Design \\
\hline 398 & Michaelsen,K. F. Nutrition and growth during infancy. The Copenhagen Cohort Study. Acta Paediatr Suppl.1997;420:1-36. & Design, IV \\
\hline 399 & $\begin{array}{l}\text { Michels, K. B.,Willett, W. C.,Graubard, B. I.,Vaidya, R. L.,Cantwell, M. M.,Sansbury, L. B.,Forman, M. R. A longitudinal study of } \\
\text { infant feeding and obesity throughout life course. Int J Obes (Lond).2007;31:1078-85. }\end{array}$ & IV \\
\hline 400 & $\begin{array}{l}\text { Mihrshahi, S.,Battistutta, D.,Magarey, A.,Daniels, L. A. Determinants of rapid weight gain during infancy: baseline results from the } \\
\text { NOURISH randomised controlled trial. BMC Pediatr.2011;11:99. }\end{array}$ & Design \\
\hline 401 & $\begin{array}{l}\text { Millar, L.,Kremer, P.,de Silva-Sanigorski, A.,McCabe, M. P.,Mavoa, H.,Moodie, M.,Utter, J.,Bell, C.,Malakellis, M.,Mathews, } \\
\text { L.,Roberts, G.,Robertson, N.,Swinburn, B. A. Reduction in overweight and obesity from a 3-year community-based intervention in } \\
\text { Australia: the 'It's Your Move!' project. Obes Rev.2011;12 Suppl 2:20-8. }\end{array}$ & IV, Age \\
\hline 402 & $\begin{array}{l}\text { Mimouni-Bloch, A.,Kachevanskaya, A.,Mimouni, F. B.,Shuper, A., Raveh, E.,Linder, N. Breastfeeding may protect from developing } \\
\text { attention-deficit/hyperactivity disorder. Breastfeed Med.2013;8:363-7. }\end{array}$ & IV \\
\hline 403 & $\begin{array}{l}\text { Mittal, S.,Gupta, M. C. Evaluation of a supplementary feeding programme through take home system. J Trop Pediatr.1980;26:50- } \\
\text { 3. }\end{array}$ & IV \\
\hline 404 & $\begin{array}{l}\text { Miura T,Fukuda M,Mizuno M,Ohte N. Need for public awareness regarding low birth weight and bottle feeding. Hypertens } \\
\text { Res.2016. }\end{array}$ & Design \\
\hline 405 & $\begin{array}{l}\text { Molgaard, C.,Larnkjaer, A.,Mark, A. B.,Michaelsen, K. F. Are early growth and nutrition related to bone health in adolescence? } \\
\text { The Copenhagen Cohort Study of infant nutrition and growth. Am J Clin Nutr.2011;94:1865s-1869s. }\end{array}$ & IV \\
\hline 406 & $\begin{array}{l}\text { Moller, L. M.,de Hoog, M. L.,van Eijsden, M.,Gemke, R. J.,Vrijkotte, T. G. Infant nutrition in relation to eating behaviour and fruit } \\
\text { and vegetable intake at age } 5 \text { years. Br J Nutr.2013;109:564-71. }\end{array}$ & DV \\
\hline
\end{tabular}




\begin{tabular}{|c|c|c|}
\hline 407 & $\begin{array}{l}\text { Monterrosa EC,Frongillo EA,Neufeld LM,Egan KA,Ramakrishnan U,Rasmussen KM. Maternal pre-pregnancy body mass index is } \\
\text { not associated with infant and young child feeding in low-income Mexican children 1-24 months old. Matern Child } \\
\text { Nutr.2015;11:215-28. }\end{array}$ & DV \\
\hline 408 & $\begin{array}{l}\text { Monterrosa,E. C.,Frongillo,E. A.,Vasquez-Garibay,E. M.,Romero-Velarde,E.,Casey,L. M.,Willows,N. D. Predominant breast- } \\
\text { feeding from birth to six months is associated with fewer gastrointestinal infections and increased risk for iron deficiency among } \\
\text { infants. J Nutr.2008;138:1499-504. }\end{array}$ & IV \\
\hline 409 & $\begin{array}{l}\text { Monti G,Viola S,Baro C,Cresi F,Tovo PA,Moro G,Ferrero MP,Conti A,Bertino E. Tolerability of donkey's milk in } 92 \text { highly- } \\
\text { problematic cow's milk allergic children. J Biol Regul Homeost Agents.2012;26:75-82. }\end{array}$ & IV \\
\hline 410 & $\begin{array}{l}\text { Moore,S. E.,Prentice,A. M.,Wagatsuma,Y.,Fulford,A. J.,Collinson,A. C.,Raqib,R.,Vahter,M.,Persson,L. A.,Arifeen,S. E. Early-life } \\
\text { nutritional and environmental determinants of thymic size in infants born in rural Bangladesh. Acta Paediatr.2009;98:1168-75. }\end{array}$ & IV, DV \\
\hline 411 & Moreno M. Early infant feeding and obesity risk. JAMA Pediatr.2014;168:1084. & Design \\
\hline 412 & Morgan JB,Mumford PM. A follow-up study of nutrition and anthropometry in pre-school children. Proc Nutr Soc.1980;39:5a. & Design \\
\hline 413 & $\begin{array}{l}\text { Morgan, J. B.,Lucas, A.,Fewtrell, M. S. Does weaning influence growth and health up to } 18 \text { months?. Archives of Disease in } \\
\text { Childhood: Education and Practice Edition.2004;89:728-733. }\end{array}$ & Design \\
\hline 414 & $\begin{array}{l}\text { Morley,R.,Abbott,R.,Fairweather-Tait,S.,MacFadyen,U.,Stephenson,T.,Lucas,A. Iron fortified follow on formula from } 9 \text { to } 18 \\
\text { months improves iron status but not development or growth: a randomised trial. Arch Dis Child.1999;81:247-52. }\end{array}$ & IV \\
\hline 415 & $\begin{array}{l}\text { Morrow-Tlucak, M.,Haude, R. H.,Ernhart, C. B. Breastfeeding and cognitive development in the first } 2 \text { years of life. Soc Sci } \\
\text { Med.1988;26:635-9. }\end{array}$ & IV \\
\hline 416 & Morton,R. E.,Nysenbaum,A.,Price,K. Iron status in the first year of life. J Pediatr Gastroenterol Nutr.1988;7:707-12. & DV \\
\hline 417 & $\begin{array}{l}\text { Mumford, P.,Morgan, J. B. A longitudinal study of nutrition and growth of infants initially on the upper and lower centile for weight } \\
\text { and age. Int J Obes.1982;6:335-41. }\end{array}$ & IV, DV \\
\hline 418 & $\begin{array}{l}\text { Musaad, S. M.,Donovan, S. M.,Fiese, B. H. Parental perception of child weight in the first two years-of-life: a potential link } \\
\text { between infant feeding and preschoolers' diet. Appetite.2015;91:90-100. }\end{array}$ & $\begin{array}{l}\text { Design, } \\
\text { DV }\end{array}$ \\
\hline 419 & $\begin{array}{l}\text { Nagahara, K.,Dobashi, K.,Itabashi, K. Feeding choice has a gender-associated effect on infant growth. Pediatr Int.2013;55:481- } \\
7 .\end{array}$ & IV \\
\hline 420 & $\begin{array}{l}\text { Nakao, R. M. Effects of an education program on the health and illness profile of rural breast-fed babies. Philipp J } \\
\text { Nurs.1988;58:12-8. }\end{array}$ & IV \\
\hline 421 & $\begin{array}{l}\text { Narese, F.,Puccio, G.,Mazzucco, W.,Falzone, A.,Venturella, V.,Narese, D.,Capra, E. Earlier appearance of the ossification center } \\
\text { of the femoral head in breast-fed versus formula-fed infants. Nutrition.2011;27:1108-11. }\end{array}$ & Design, IV \\
\hline 422 & $\begin{array}{l}\text { Nauta,A. J.,Garssen,J. Nutritional programming of immune defense against infections in early life. Nutricia Research, Utrecht, } \\
\text { Netherlands.2014;12:113-126. }\end{array}$ & Design \\
\hline 423 & $\begin{array}{l}\text { Newman,J. E.,Garces,A.,Mazariegos,M.,Michael Hambidge,K.,Manasyan,A.,Tshefu,A.,Lokangaka,A.,Sami,N.,Carlo,W. } \\
\text { A.,Bose,C. L.,Pasha,O.,Goco,N.,Chomba,E.,Goldenberg,R. L.,Wright,L. L.,Koso-Thomas,M.,Krebs,N. F. Theory-driven process }\end{array}$ & Design, \\
\hline
\end{tabular}




\begin{tabular}{|c|c|c|}
\hline & evaluation of a complementary feeding trial in four countries. Health Educ Res.2014;29:297-305. & DV \\
\hline 424 & $\begin{array}{l}\text { Ng, S. C.,Chong, Y. S.,Rauff, M.,Myo, Z. M.,Nurfarah, C.,Deurenberg, P. R. The influence of breast feeding compared to formula } \\
\text { feeding on infant adiposity. Ann Acad Med Singapore.2004;33:S75. }\end{array}$ & Design, IV \\
\hline 425 & $\begin{array}{l}\text { Nguyen, N. D.,Allen, J. R.,Peat, J. K.,Schofield, W. N.,Nossar, V.,Eisenbruch, M.,Gaskin, K. J. Growth and feeding practices of } \\
\text { Vietnamese infants in Australia. Eur J Clin Nutr.2004;58:356-62. }\end{array}$ & IV, DV \\
\hline 426 & $\begin{array}{l}\text { Nielsen GA,Thomsen BL,Michaelsen KF. Influence of breastfeeding and complementary food on growth between } 5 \text { and } 10 \\
\text { months. Acta Paediatr.1998;87:911-7. }\end{array}$ & Design \\
\hline 427 & $\begin{array}{l}\text { Niinikoski, H.,Lagstrom, H.,Jokinen, E.,Siltala, M.,Ronnemaa, T.,Viikari, J.,Raitakari, O. T.,Jula, A.,Marniemi, J.,Nanto-Salonen, } \\
\text { K.,Simell, O. Impact of repeated dietary counseling between infancy and } 14 \text { years of age on dietary intakes and serum lipids and } \\
\text { lipoproteins: the STRIP study. Circulation.2007;116:1032-40. }\end{array}$ & IV \\
\hline 428 & $\begin{array}{l}\text { Northstone, K.,Emmett, P.,Nethersole, F. The effect of age of introduction to lumpy solids on foods eaten and reported feeding } \\
\text { difficulties at } 6 \text { and } 15 \text { months. J Hum Nutr Diet.2001;14:43-54. }\end{array}$ & DV \\
\hline 429 & $\begin{array}{l}\text { Novotny, R.,Coleman, P.,Tenorio, L.,Davison, N.,Camacho, T.,Ramirez, V.,Vijayadeva, V.,Untalan, P.,Tudela, M. D. } \\
\text { Breastfeeding is associated with lower body mass index among children of the Commonwealth of the Northern Mariana Islands. } \\
\text { J Am Diet Assoc.2007;107:1743-6. }\end{array}$ & Design, IV \\
\hline 430 & $\begin{array}{l}\text { Ntouva, A., Rogers, I.,MacAdam, A.,Emmett, P. Weaning practices and iron status of exclusively breast fed infants. Journal of } \\
\text { Human Nutrition \& Dietetics.2011;24:297-298 2p. }\end{array}$ & Design \\
\hline 431 & $\begin{array}{l}\text { Nwaru BI,Takkinen HM,Niemela O,Kaila M,Erkkola M,Ahonen S,Haapala AM,Kenward MG,Pekkanen J,Lahesmaa R,Kere } \\
\text { J,Simell O,Veijola R,Ilonen J,Hyoty H,Knip M,Virtanen SM. Timing of infant feeding in relation to childhood asthma and allergic } \\
\text { diseases. J Allergy Clin Immunol.2013;131:78-86. }\end{array}$ & DV \\
\hline 432 & Obatolu, V. A. Growth pattern of infants fed with a mixture of extruded malted maize and cowpea. Nutrition.2003;19:174-8. & Country \\
\hline 433 & $\begin{array}{l}\text { Oddy, W. H.,Kendall, G. E.,Blair, E.,De Klerk, N. H.,Stanley, F. J.,Landau, L. I.,Silburn, S.,Zubrick, S. Breast feeding and } \\
\text { cognitive development in childhood: a prospective birth cohort study. Paediatr Perinat Epidemiol.2003;17:81-90. }\end{array}$ & IV \\
\hline 434 & $\begin{array}{l}\text { Oddy, W. H.,Robinson, M.,Kendall, G. E.,Li, J.,Zubrick, S. R.,Stanley, F. J. Breastfeeding and early child development: a } \\
\text { prospective cohort study. Acta Paediatr.2011;100:992-9. }\end{array}$ & IV \\
\hline 435 & $\begin{array}{l}\text { Oddy, W. H.,Sherriff, J. L. Breastfeeding, body mass index, asthma and atopy in children. Asia Pac J Public Health.2003;15 } \\
\text { Suppl:S15-7. }\end{array}$ & IV \\
\hline 436 & $\begin{array}{l}\text { O'Donovan,S. M.,O'B Hourihane J,Murray,D. M.,Kenny,L. C.,Khashan,A. S.,Chaoimh,C. N.,Irvine,A. D.,Kiely,M. Neonatal } \\
\text { adiposity increases the risk of atopic dermatitis during the first year of life. J Allergy Clin Immunol.2015. }\end{array}$ & DV \\
\hline 437 & $\begin{array}{l}\text { Oelofse,A.,Van Raaij,J. M.,Benade,A. J.,Dhansay,M. A.,Tolboom,J. J.,Hautvast,J. G. The effect of a micronutrient-fortified } \\
\text { complementary food on micronutrient status, growth and development of 6- to 12-month-old disadvantaged urban South African } \\
\text { infants. Int J Food Sci Nutr.2003;54:399-407. }\end{array}$ & Country \\
\hline 438 & Ong, K. K.,Emmett, P. M.,Noble, S.,Ness, A.,Dunger, D. B. Dietary energy intake at the age of 4 months predicts postnatal weight & IV \\
\hline
\end{tabular}




\begin{tabular}{|c|c|c|}
\hline & gain and childhood body mass index. Pediatrics.2006;117:e503-8. & \\
\hline 439 & $\begin{array}{l}\text { Ong, K. K.,Preece, M. A.,Emmett, P. M.,Ahmed, M. L.,Dunger, D. B. Size at birth and early childhood growth in relation to } \\
\text { maternal smoking, parity and infant breast-feeding: longitudinal birth cohort study and analysis. Pediatr Res.2002;52:863-7. }\end{array}$ & IV \\
\hline 440 & $\begin{array}{l}\text { Onyango, A. W.,Borghi, E.,de Onis, M.,Casanovas Mdel, C.,Garza, C. Complementary feeding and attained linear growth among } \\
\text { 6-23-month-old children. Public Health Nutr.2014;17:1975-83. }\end{array}$ & Design \\
\hline 441 & $\begin{array}{l}\text { Ou, X.,Andres, A.,Cleves, M. A.,Pivik, R. T.,Snow, J. H.,Ding, Z.,Badger, T. M. Sex-specific association between infant diet and } \\
\text { white matter integrity in 8-y-old children. Pediatr Res.2014;76:535-43. }\end{array}$ & IV \\
\hline 442 & $\begin{array}{l}\text { Ounsted, M. K.,Moar, V. A.,Scott, A. Large-for-dates babies at the age of four years: health, handicap and developmental status. } \\
\text { Early Hum Dev.1983;9:9-19. }\end{array}$ & IV \\
\hline 443 & Pachucki MA. Food pattern analysis over time: unhealthful eating trajectories predict obesity. Int J Obes (Lond).2012;36:686-94. & Age \\
\hline 444 & $\begin{array}{l}\text { Parry, J. E.,Ip, D. K.,Chau, P. Y.,Wu, K. M.,Tarrant, M. Predictors and consequences of in-hospital formula supplementation for } \\
\text { healthy breastfeeding newborns. J Hum Lact.2013;29:527-36. }\end{array}$ & IV, DV \\
\hline 445 & Parsons, T. J.,Power, C.,Manor, O. Infant feeding and obesity through the lifecourse. Arch Dis Child.2003;88:793-4. & IV \\
\hline 446 & $\begin{array}{l}\text { Patsourou, A.,Konstantinides, T.,Mantadakis, E.,Tsalkidis, A.,Zarras, C.,Balaska, A.,Simopoulos, K.,Chatzimichael, A. Growth of } \\
\text { exclusively breastfed and self-weaned children of Greece aged 0-36 months. Breastfeed Med.2012;7:521-5. }\end{array}$ & IV \\
\hline 447 & $\begin{array}{l}\text { Paul IM,Savage JS,Anzman SL,Beiler JS,Marini ME,Stokes JL,Birch LL. Preventing obesity during infancy: a pilot study. Obesity } \\
\text { (Silver Spring).2011;19:353-61. }\end{array}$ & IV \\
\hline 448 & Paul, A.,Whitehead, R. Infant feeding: the weighting game. Community Outlook.1986:11-7. & Design \\
\hline 449 & Paul, S. P.,Smith, B. A.,Taylor, T. M.,Walker, J. Take with a grain of salt. Clin Chem.2013;59:348-51. & Design \\
\hline 450 & $\begin{array}{l}\text { Peat,J. K.,Mihrshahi,S.,Kemp,A. S.,Marks,G. B.,Tovey,E. R.,Webb,K.,Mellis,C. M.,Leeder,S. R. Three-year outcomes of dietary } \\
\text { fatty acid modification and house dust mite reduction in the Childhood Asthma Prevention Study. J Allergy Clin } \\
\text { Immunol.2004;114:807-13. }\end{array}$ & IV, DV \\
\hline 451 & $\begin{array}{l}\text { Penny,M. E.,Creed-Kanashiro,H. M.,Robert,R. C.,Narro,M. R.,Caulfield,L. E.,Black,R. E. Effectiveness of an educational } \\
\text { intervention delivered through the health services to improve nutrition in young children: a cluster-randomised controlled trial. } \\
\text { Lancet.2005;365:1863-72. }\end{array}$ & IV \\
\hline 452 & $\begin{array}{l}\text { Penrod,J. C.,Anderson,K.,Acosta,P. B. Impact on iron status of introducing cow's milk in the second six months of life. J Pediatr } \\
\text { Gastroenterol Nutr.1990;10:462-7. }\end{array}$ & IV, DV \\
\hline 453 & $\begin{array}{l}\text { Perez-Escamilla, R.,Cohen, R. J.,Brown, K. H.,Rivera, L. L.,Canahuati, J.,Dewey, K. G. Maternal anthropometric status and } \\
\text { lactation performance in a low-income Honduran population: evidence for the role of infants. Am J Clin Nutr.1995;61:528-34. }\end{array}$ & IV, DV \\
\hline 454 & Persson LA. Infant feeding and growth--a longitudinal study in three Swedish communities. Ann Hum Biol.1985;12:41-52. & IV \\
\hline
\end{tabular}




\begin{tabular}{|c|c|c|}
\hline 455 & $\begin{array}{l}\text { Persson,L. A.,Lundstrom,M.,Lonnerdal,B.,Hernell,O. Are weaning foods causing impaired iron and zinc status in 1-year-old } \\
\text { Swedish infants? A cohort study. Acta Paediatr.1998;87:618-22. }\end{array}$ & IV, DV \\
\hline 456 & $\begin{array}{l}\text { Peters,R. L.,Allen,K. J.,Dharmage,S. C.,Lodge,C. J.,Koplin,J. J.,Ponsonby,A. L.,Wake,M.,Lowe,A. J.,Tang,M. L.,Matheson,M. } \\
\text { C.,Gurrin,L. C. Differential factors associated with challenge-proven food allergy phenotypes in a population cohort of infants: a } \\
\text { latent class analysis. Clin Exp Allergy.2015;45:953-63. }\end{array}$ & DV \\
\hline 457 & $\begin{array}{l}\text { Pfluger M,Winkler C,Hummel S,Ziegler AG. Early infant diet in children at high risk for type } 1 \text { diabetes. Horm Metab } \\
\text { Res.2010;42:143-8. }\end{array}$ & IV \\
\hline 458 & $\begin{array}{l}\text { Pham,V. P.,Nguyen,V. H.,Salvignol,B.,Treche,S.,Wieringa,F. T.,Dijkhuizen,M. A.,Nguyen,C. K.,Pham,D. T.,Schwartz,H.,Berger,J. } \\
\text { A six-month intervention with two different types of micronutrient-fortified complementary foods had distinct short- and long-term } \\
\text { effects on linear and ponderal growth of Vietnamese infants. J Nutr.2012;142:1735-40. }\end{array}$ & Country \\
\hline 459 & $\begin{array}{l}\text { Phu,P. V.,Hoan,N. V.,Salvignol,B.,Treche,S.,Wieringa,F. T.,Khan,N. C.,Tuong,P. D.,Berger,J. Complementary foods fortified with } \\
\text { micronutrients prevent iron deficiency and anemia in Vietnamese infants. J Nutr.2010;140:2241-7. }\end{array}$ & DV \\
\hline 460 & $\begin{array}{l}\text { Piemontese, P.,Gianni, M. L.,Braegger, C. P.,Chirico, G.,Gruber, C., Riedler, J.,Arslanoglu, S.,van Stuijvenberg, M.,Boehm, } \\
\text { G.,Jelinek, J.,Roggero, P. Tolerance and safety evaluation in a large cohort of healthy infants fed an innovative prebiotic formula: } \\
\text { a randomized controlled trial. PLoS One.2011;6:e28010. }\end{array}$ & IV \\
\hline 461 & $\begin{array}{l}\text { Pimpin, L.,Jebb, S.,Johnson, L.,Wardle, J.,Ambrosini, G. L. Dietary protein intake is associated with body mass index and weight } \\
\text { up to } 5 \text { y of age in a prospective cohort of twins. Am J Clin Nutr.2016;103:389-97. }\end{array}$ & IV \\
\hline 462 & $\begin{array}{l}\text { Pivik, R. T.,Andres, A.,Badger, T. M. Effects of diet on early stage cortical perception and discrimination of syllables differing in } \\
\text { voice-onset time: a longitudinal ERP study in } 3 \text { and } 6 \text { month old infants. Brain Lang.2012;120:27-41. }\end{array}$ & IV \\
\hline 463 & $\begin{array}{l}\text { Pivik, R. T.,Dykman, R. A.,Jing, H.,Gilchrist, J. M.,Badger, T. M. The influence of infant diet on early developmental changes in } \\
\text { processing human voice speech stimuli: ERP variations in breast and milk formula-fed infants at } 3 \text { and } 6 \text { months after birth. Dev } \\
\text { Neuropsychol.2007;31:279-335. }\end{array}$ & IV \\
\hline 464 & $\begin{array}{l}\text { Piwoz, E. G.,Black, R. E.,Lopez de Romana, G.,Creed de Kanashiro, H.,Brown, K. H. The relationship between infants' preceding } \\
\text { appetite, illness, and growth performance and mothers' subsequent feeding practice decisions. Soc Sci Med.1994;39:851-60. }\end{array}$ & IV, DV \\
\hline 465 & $\begin{array}{l}\text { Piwoz, E. G.,Creed de Kanashiro, H.,Lopez de Romana, G.,Black, R. E.,Brown, K. H. Within- and between-individual variation in } \\
\text { energy intakes by low-income Peruvian infants. Eur J Clin Nutr.1994;48:333-40. }\end{array}$ & IV, DV \\
\hline 466 & $\begin{array}{l}\text { Piwoz,E. G.,Lopez de Romana,G.,Creed de Kanashiro,H.,Black,R. E.,Brown,K. H. Indicators for monitoring the growth of } \\
\text { peruvian infants: weight and length gain vs attained weight and length. Am J Public Health.1994;84:1132-8. }\end{array}$ & IV \\
\hline 467 & $\begin{array}{l}\text { Plagemann, A.,Harder, T.,Kohlhoff, R.,Fahrenkrog, S.,Rodekamp, E.,Franke, K.,Dudenhausen, J. W. Impact of early neonatal } \\
\text { breast-feeding on psychomotor and neuropsychological development in children of diabetic mothers. Diabetes } \\
\text { Care.2005;28:573-8. }\end{array}$ & IV \\
\hline 468 & $\begin{array}{l}\text { Plonka, K. A.,Pukallus, M. L.,Barnett, A.,Holcombe, T. F.,Walsh, L. J.,Seow, W. K. A controlled, longitudinal study of home visits } \\
\text { compared to telephone contacts to prevent early childhood caries. Int J Paediatr Dent.2013;23:23-31. }\end{array}$ & DV \\
\hline 469 & $\begin{array}{l}\text { Poh,Bee Koon,Ng,Boon Koon,Siti Haslinda,Mohd Din,Nik Shanita,Safii,Wong,Jyh Eiin,Budin,Siti Balkis,Ruzita,Abd Talib,Ng,Lai } \\
\text { Oon,Khouw,Ilse,Norimah,A. Karim. Nutritional status and dietary intakes of children aged } 6 \text { months to } 12 \text { years: findings of the }\end{array}$ & Design, IV \\
\hline
\end{tabular}




\begin{tabular}{|c|c|c|}
\hline & Nutrition Survey of Malaysian Children (SEANUTS Malaysia). British Journal of Nutrition.2013;110:S21-35 1p. & \\
\hline 470 & $\begin{array}{l}\text { Polańska, K.,Muszyński, P.,Sobala, W.,Dziewirska, E.,Merecz-Kot, D.,Hanke, W. Maternal lifestyle during pregnancy and child } \\
\text { psychomotor development - Polish Mother and Child Cohort study. Early Human Development.2015;91:317-325. }\end{array}$ & IV \\
\hline 471 & $\begin{array}{l}\text { Pollitt, E.,Gorman, K. S.,Engle, P. L.,Rivera, J. A.,Martorell, R. Nutrition in early life and the fulfillment of intellectual potential. J } \\
\text { Nutr.1995;125:1111s-1118s. }\end{array}$ & Design, IV \\
\hline 472 & $\begin{array}{l}\text { Pollitt, E.,Watkins, W. E.,Husaini, M. A. Three-month nutritional supplementation in Indonesian infants and toddlers benefits } \\
\text { memory function } 8 \text { y later. Am J Clin Nutr.1997;66:1357-63. }\end{array}$ & Country \\
\hline 473 & Potur, A. H.,Kalmaz, N. An investigation into feeding errors of 0-4-month-old infants. J Trop Pediatr.1995;41:120-2. & $\begin{array}{l}\text { Design, } \\
\text { IV,DV }\end{array}$ \\
\hline 474 & Potur, A. H.,Kalmaz, N. An investigation into feeding errors of 0-4-month-old infants. J Trop Pediatr.1996;42:173-5. & $\begin{array}{l}\text { Design, } \\
\text { IV,DV }\end{array}$ \\
\hline 475 & $\begin{array}{l}\text { Prentice P,Koulman A,Matthews L,Acerini CL,Ong KK,Dunger DB. Lipidomic analyses, breast- and formula-feeding, and growth } \\
\text { in infants. J Pediatr.2015;166:276-81.e6. }\end{array}$ & IV \\
\hline 476 & $\begin{array}{l}\text { Pridham, K. F. Infant feeding and anticipatory care: supporting the adaptation of parents to their new babies. Matern Child Nurs } \\
\text { J.1981;10:111-26. }\end{array}$ & Design \\
\hline 477 & $\begin{array}{l}\text { Priego, T.,Sanchez, J.,Pico, C.,Ahrens, W.,Bammann, K.,De Henauw, S.,Fraterman, A.,lacoviello, L.,Lissner, L.,Molnar, } \\
\text { D.,Moreno, L. A.,Siani, A.,Tornaritis, M.,Veidebaum, T.,Palou, A. Influence of breastfeeding on blood-cell transcript-based } \\
\text { biomarkers of health in children. Pediatr Obes.2014;9:463-70. }\end{array}$ & IV \\
\hline 478 & $\begin{array}{l}\text { Prodam, F.,Roccio, M.,Trovato, L.,Ricotti, R.,Moia, S.,Giglione, E.,Petri, A., Walker, G. E.,Bellone, S.,Bona, G. Adiponectin } \\
\text { oligomers are similarly distributed in adequate-for-gestational-age obese children irrespective of feeding in their first year. Pediatr } \\
\text { Res.2015;77:808-13. }\end{array}$ & IV, DV \\
\hline 479 & $\begin{array}{l}\text { Puri, R.,Chawla, P.,Sharma, M.,Pershad, D. Impact of an on-going supplementary feeding programme on the mental abilities of } \\
\text { children. Indian J Pediatr.1984;51:653-7. }\end{array}$ & IV \\
\hline 480 & $\begin{array}{l}\text { Purwestri, R. C.,Scherbaum, V.,Inayati, D. A.,Wirawan, N. N.,Suryantan, J.,Bloem, M. A.,Pangaribuan, R. V.,Stuetz, } \\
\text { W.,Hoffmann, V.,Qaim, M.,Biesalski, H. K.,Bellows, A. C. Supplementary feeding with locally-produced Ready-to-Use Food } \\
\text { (RUF) for mildly wasted children on Nias Island, Indonesia: comparison of daily and weekly program outcomes. Asia Pac J Clin } \\
\text { Nutr.2012;21:374-9. }\end{array}$ & $\begin{array}{l}\text { Health } \\
\text { status, IV }\end{array}$ \\
\hline 481 & $\begin{array}{l}\text { Qureshi, B.,Morgan, J. B.,Kimer, A. C.,Donaldson, D.,Dickerson, J. W. Feeding practices and birth weights of infants in Southall, } \\
\text { Middlesex. J R Soc Health.1988;108:77-80. }\end{array}$ & IV, DV \\
\hline 482 & $\begin{array}{l}\text { Radhakrishna,K. V.,Hemalatha,R.,Geddam,J. J.,Kumar,P. A.,Balakrishna,N.,Shatrugna,V. Effectiveness of zinc supplementation } \\
\text { to full term normal infants: a community based double blind, randomized, controlled, clinical trial. PLoS One.2013;8:e61486. }\end{array}$ & IV \\
\hline 483 & $\begin{array}{l}\text { Ramokolo, V.,Lombard, C.,Chhagan, M.,Engebretsen, I. M.,Doherty, T.,Goga, A. E.,Fadnes, L. T.,Zembe, W.,Jackson, D. J.,Van } \\
\text { den Broeck, J. Effects of early feeding on growth velocity and overweight/obesity in a cohort of HIV unexposed South African } \\
\text { infants and children. Int Breastfeed J.2015;10:14. }\end{array}$ & IV \\
\hline
\end{tabular}




\begin{tabular}{|c|c|c|}
\hline 484 & $\begin{array}{l}\text { Rao,S.,Rajpathak,V. Breastfeeding and weaning practices in relation to nutritional status of infants. Indian Pediatr.1992;29:1533- } \\
\text { 9. }\end{array}$ & IV, DV \\
\hline 485 & Rapley, G. Talking about weaning. Community Pract.2011;84:40-1. & Design \\
\hline 486 & $\begin{array}{l}\text { Rask-Nissila,L.,Jokinen,E.,Terho,P.,Tammi,A.,Hakanen,M.,Ronnemaa,T.,Viikari,J.,Seppanen,R.,Valimaki,I.,Helenius,H.,Simell,O. } \\
\text { Effects of diet on the neurologic development of children at } 5 \text { years of age: the STRIP project. J Pediatr.2002;140:328-33. }\end{array}$ & IV \\
\hline 487 & $\begin{array}{l}\text { Ravelli, A. C.,van der Meulen, J. H.,Osmond, C.,Barker, D. J.,Bleker, O. P. Infant feeding and adult glucose tolerance, lipid } \\
\text { profile, blood pressure, and obesity. Arch Dis Child.2000;82:248-52. }\end{array}$ & IV \\
\hline 488 & $\begin{array}{l}\text { Reifsnider, E. Reversing growth deficiency in children: the effect of a community-based intervention. J Pediatr Health } \\
\text { Care.1998;12:305-12. }\end{array}$ & $\begin{array}{l}\text { Health } \\
\text { status, IV }\end{array}$ \\
\hline 489 & $\begin{array}{l}\text { Requejo,A. M.,Navia,B.,Ortega,R. M.,Lopez-Sobaler,A. M.,Quintas,E.,Gaspar,M. J.,Osorio,O. The age at which meat is first } \\
\text { included in the diet affects the incidence of iron deficiency and ferropenic anaemia in a group of pre-school children from Madrid. } \\
\text { Int J Vitam Nutr Res.1999;69:127-31. }\end{array}$ & Design \\
\hline 490 & $\begin{array}{l}\text { Richards, M.,Wadsworth, M.,Rahimi-Foroushani, A.,Hardy, R.,Kuh, D.,Paul, A. Infant nutrition and cognitive development in the } \\
\text { first offspring of a national UK birth cohort. Dev Med Child Neurol.1998;40:163-7. }\end{array}$ & IV \\
\hline 491 & $\begin{array}{l}\text { Rifas-Shiman SL,Sherry B,Scanlon K,Birch LL,Gillman MW,Taveras EM. Does maternal feeding restriction lead to childhood } \\
\text { obesity in a prospective cohort study?. Arch Dis Child.2011;96:265-9. }\end{array}$ & IV \\
\hline 492 & $\begin{array}{l}\text { Rios-Castillo, I.,Cerezo, S.,Corvalan, C.,Martinez, M.,Kain, J. Risk factors during the prenatal period and the first year of life } \\
\text { associated with overweight in 7-year-old low-income Chilean children. Matern Child Nutr.2015;11:595-605. }\end{array}$ & Design \\
\hline 493 & $\begin{array}{l}\text { Rivera,J. A.,Habicht,J. P. Effect of supplementary feeding on the prevention of mild-to-moderate wasting in conditions of endemic } \\
\text { malnutrition in Guatemala. Bull World Health Organ.2002;80:926-32. }\end{array}$ & IV \\
\hline 494 & Roberts, D. W. Growth of breast fed and bottle fed infants. N Z Med J.1980;92:45-6. & IV \\
\hline 495 & $\begin{array}{l}\text { Robinson, S. M.,Simmonds, S. J.,Jameson, K. A.,Syddall, H. E.,Dennison, E. M.,Cooper, C.,Sayer, A. A. Muscle strength in older } \\
\text { community-dwelling men is related to type of milk feeding in infancy. J Gerontol A Biol Sci Med Sci.2012;67:990-6. }\end{array}$ & IV \\
\hline 496 & $\begin{array}{l}\text { Robinson, S.,Ntani, G.,Simmonds, S.,Syddall, H.,Dennison, E.,Sayer, A. A.,Barker, D.,Cooper, C. Type of milk feeding in infancy } \\
\text { and health behaviours in adult life: findings from the Hertfordshire Cohort Study. Br J Nutr.2013;109:1114-22. }\end{array}$ & IV, DV \\
\hline 497 & Rogan, W. J.,Gladen, B. C. Breast-feeding and cognitive development. Early Hum Dev.1993;31:181-93. & IV \\
\hline 498 & $\begin{array}{l}\text { Rolland-Cachera,M. F.,Maillot,M.,Deheeger,M.,Souberbielle,J. C.,Peneau,S.,Hercberg,S. Association of nutrition in early life with } \\
\text { body fat and serum leptin at adult age. Int J Obes (Lond).2013;37:1116-22. }\end{array}$ & IV \\
\hline 499 & $\begin{array}{l}\text { Rosado,J. L.,Lopez,P.,Garcia,O. P.,Alatorre,J.,Alvarado,C. Effectiveness of the nutritional supplement used in the Mexican } \\
\text { Oportunidades programme on growth, anaemia, morbidity and cognitive development in children aged 12-24 months. Public } \\
\text { Health Nutr.2011;14:931-7. }\end{array}$ & IV \\
\hline 500 & Routi, T.,Ronnemaa, T.,Lapinleimu, H.,Salo, P.,Viikari, J.,Leino, A.,Valimaki, I.,Jokinen, E.,Simell, O. Effect of weaning on serum & IV, DV \\
\hline
\end{tabular}




\begin{tabular}{|c|c|c|}
\hline & lipoprotein(a) concentration: the STRIP baby study. Pediatr Res.1995;38:522-7. & \\
\hline 501 & $\begin{array}{l}\text { Rowland, M. G. The "why" and "when" of introducing food to infants: growth in young breast-fed infants and some nutritional } \\
\text { implications. Am J Clin Nutr.1985;41:459-63. }\end{array}$ & Design \\
\hline 502 & $\begin{array}{l}\text { Roy, S. K.,Fuchs, G. J.,Mahmud, Z.,Ara, G.,Islam, S.,Shafique, S.,Akter, S. S.,Chakraborty, B. Intensive nutrition education with } \\
\text { or without supplementary feeding improves the nutritional status of moderately-malnourished children in Bangladesh. J Health } \\
\text { Popul Nutr.2005;23:320-30. }\end{array}$ & $\begin{array}{l}\text { Health } \\
\text { status }\end{array}$ \\
\hline 503 & $\begin{array}{l}\text { Rudnicka, A. R., Owen, C. G.,Richards, M.,Wadsworth, M. E.,Strachan, D. P. Effect of breastfeeding and sociodemographic } \\
\text { factors on visual outcome in childhood and adolescence. Am J Clin Nutr.2008;87:1392-9. }\end{array}$ & IV \\
\hline 504 & Rudy,C. A. Vegetarian diets for children. Pediatr Nurs.1984;10:329-33. & Design \\
\hline 505 & $\begin{array}{l}\text { Ruel, M. T.,Rivera, J.,Habicht, J. P.,Martorell, R. Differential response to early nutrition supplementation: long-term effects on } \\
\text { height at adolescence. Int J Epidemiol.1995;24:404-12. }\end{array}$ & IV, Date \\
\hline 506 & $\begin{array}{l}\text { Russell, C. G.,Taki, S.,Azadi, L.,Campbell, K. J.,Laws, R.,Elliott, R.,Denney-Wilson, E. A qualitative study of the infant feeding } \\
\text { beliefs and behaviours of mothers with low educational attainment. BMC Pediatr.2016;16:69. }\end{array}$ & DV \\
\hline 507 & Ryan, A. S.,Gussler, J. D. Biocultural factors affecting infant feeding and growth. Med Anthropol.1985;9:93-5. & Design \\
\hline 508 & $\begin{array}{l}\text { Rzehak, P.,Sausenthaler, S.,Koletzko, S., Reinhardt, D.,von Berg, A.,Kramer, U.,Berdel, D.,Bollrath, C.,Grubl, A.,Bauer, C. } \\
\text { P.,Wichmann, H. E.,Heinrich, J. Long-term effects of hydrolyzed protein infant formulas on growth--extended follow-up to } 10 \text { y of } \\
\text { age: results from the German Infant Nutritional Intervention (GINI) study. Am J Clin Nutr.2011;94:1803s-1807s. }\end{array}$ & IV \\
\hline 509 & $\begin{array}{l}\text { Saco-Pollitt, C.,Triana, N.,Harahap, H.,Husaini, M.,Jahari, A. B.,Pollitt, E. The eco-cultural context of the undernourished children } \\
\text { in a study on the effects of early supplementary feeding in Indonesia. Eur J Clin Nutr.2000;54 Suppl 2:S11-5. }\end{array}$ & $\begin{array}{l}\text { Design, } \\
\text { Health } \\
\text { status }\end{array}$ \\
\hline 510 & $\begin{array}{l}\text { Sacrey, L. A.,Karl, J. M.,Whishaw, I. Q. Development of rotational movements, hand shaping, and accuracy in advance and } \\
\text { withdrawal for the reach-to-eat movement in human infants aged 6-12 months. Infant Behav Dev.2012;35:543-60. }\end{array}$ & IV, DV \\
\hline 511 & $\begin{array}{l}\text { Saha, K. K.,Frongillo, E. A.,Alam, D. S.,Arifeen, S. E.,Persson, L. A., Rasmussen, K. M. Appropriate infant feeding practices result } \\
\text { in better growth of infants and young children in rural Bangladesh. Am J Clin Nutr.2008;87:1852-9. }\end{array}$ & IV \\
\hline 512 & $\begin{array}{l}\text { Samuel, T. M.,Thomas, T.,Bhat, S.,Kurpad, A. V. Are infants born in baby-friendly hospitals being exclusively breastfed until } 6 \\
\text { months of age?. Eur J Clin Nutr.2012;66:459-65. }\end{array}$ & IV \\
\hline 513 & Saner G,Dagoglu T,Uzkan I,Neyzi O. Promotion of breastfeeding in the postpartum mother. Turk J Pediatr.1985;27:63-8. & IV \\
\hline 514 & Sanger, R. G.,Bystrom, E. B. Breast feeding: does it affect oral facial growth?. Dent Hyg (Chic).1982;56:44-7. & Design \\
\hline 515 & $\begin{array}{l}\text { Santos, I. S.,Matijasevich, A.,Assuncao, M. C.,Valle, N. C.,Horta, B. L.,Goncalves, H. D.,Gigante, D. P.,Martines, J. C.,Pelto, } \\
\text { G.,Victora, C. G. Promotion of Weight Gain in Early Childhood Does Not Increase Metabolic Risk in Adolescents: A 15-Year } \\
\text { Follow-Up of a Cluster-Randomized Controlled Trial. J Nutr.2015;145:2749-55. }\end{array}$ & IV \\
\hline
\end{tabular}




\begin{tabular}{|c|c|c|}
\hline 516 & $\begin{array}{l}\text { Santos,I.,Victora,C. G.,Martines,J.,Goncalves,H.,Gigante,D. P.,Valle,N. J.,Pelto,G. Nutrition counseling increases weight gain } \\
\text { among Brazilian children. J Nutr.2001;131:2866-73. }\end{array}$ & IV \\
\hline 517 & $\begin{array}{l}\text { Satter, Ma,Jabin, Sa,Abedin, N.,Mf, Mf,Parvin, R.,Dhali, M.,Amin, Mz. Development and Evaluation of Weaning Foods Using } \\
\text { Locally Available Nutritious Fruits in Bangladesh. Malaysian Journal of Nutrition.2014;20:83-92. }\end{array}$ & $\begin{array}{l}\text { Design, IV, } \\
\text { DV }\end{array}$ \\
\hline 518 & $\begin{array}{l}\text { Savino, F.,Maccario, S.,Cresi, F.,Grasso, G.,Oggero, R.,Silvestro, L.,Mussa, G. C. Bioimpedance vector analysis in breastfed and } \\
\text { formula-fed infants in the first six months of life. Adv Exp Med Biol.2004;554:501-4. }\end{array}$ & Design, IV \\
\hline 519 & $\begin{array}{l}\text { Savino, F.,Sorrenti, M.,Benetti, S.,Lupica, M. M.,Liguori, S. A.,Oggero, R. Resistin and leptin in breast milk and infants in early } \\
\text { life. Early Hum Dev.2012;88:779-82. }\end{array}$ & Design, IV \\
\hline 520 & Sawley, L. Infant feeding. Nursing (Lond).1989;3:18-23. & Design \\
\hline 521 & $\begin{array}{l}\text { Sazawal,S.,Dhingra,P.,Dhingra,U.,Gupta,S.,Iyengar,V.,Menon,V. P.,Sarkar,A.,Black,R. E. Compliance with home-based } \\
\text { fortification strategies for delivery of iron and zinc: its effect on haematological and growth markers among 6-24 months old } \\
\text { children in north India. J Health Popul Nutr.2014;32:217-26. }\end{array}$ & Country \\
\hline 522 & $\begin{array}{l}\text { Scaglioni, S.,Agostoni, C.,Notaris, R. D.,Radaelli, G.,Radice, N.,Valenti, M.,Giovannini, M.,Riva, E. Early macronutrient intake and } \\
\text { overweight at five years of age. Int J Obes Relat Metab Disord.2000;24:777-81. }\end{array}$ & IV \\
\hline 523 & $\begin{array}{l}\text { Scheiwe, A.,Hardy, R.,Watt, R. G. Four-year follow-up of a randomized controlled trial of a social support intervention on infant } \\
\text { feeding practices. Matern Child Nutr.2010;6:328-37. }\end{array}$ & IV \\
\hline 524 & Schmalz, M.,Boos, K.,Schmalz, G.,Huntington, M. K. Failure to thrive. J Fam Pract.2009;58:539-44. & Design \\
\hline 525 & $\begin{array}{l}\text { Schmidt,M. K.,Muslimatun,S.,West,C. E.,Schultink,W.,Gross,R.,Hautvast,J. G. Nutritional status and linear growth of Indonesian } \\
\text { infants in west java are determined more by prenatal environment than by postnatal factors. J Nutr.2002;132:2202-7. }\end{array}$ & Country \\
\hline 526 & $\begin{array}{l}\text { Schroeder, D. G.,Martorell, R.,Rivera, J. A.,Ruel, M. T.,Habicht, J. P. Age differences in the impact of nutritional supplementation } \\
\text { on growth. J Nutr.1995;125:1051s-1059s. }\end{array}$ & IV \\
\hline 527 & $\begin{array}{l}\text { Schroeder, N.,Rushovich, B.,Bartlett, E.,Sharma, S.,Gittelsohn, J.,Caballero, B. Early Obesity Prevention: A Randomized Trial of } \\
\text { a Practice-Based Intervention in 0-24-Month Infants. J Obes.2015;2015:795859. }\end{array}$ & IV \\
\hline 528 & $\begin{array}{l}\text { Schwartz, R.,Vigo, A.,de Oliveira, L. D.,Justo Giugliani, E. R. The Effect of a Pro-Breastfeeding and Healthy Complementary } \\
\text { Feeding Intervention Targeting Adolescent Mothers and Grandmothers on Growth and Prevalence of Overweight of Preschool } \\
\text { Children. PLoS One.2015;10:e0131884. }\end{array}$ & IV \\
\hline 529 & $\begin{array}{l}\text { Scott, J. A.,Binns, C. W.,Graham, K. I.,Oddy, W. H. Predictors of the early introduction of solid foods in infants: results of a cohort } \\
\text { study. BMC Pediatr.2009;9:60. }\end{array}$ & DV \\
\hline 530 & $\begin{array}{l}\text { Scott, J. A.,Dashti, M.,Al-Sughayer, M.,Edwards, C. A. Timing and Determinants of the Introduction of Complementary Foods in } \\
\text { Kuwait: Results of a Prospective Cohort Study. J Hum Lact.2015;31:467-73. }\end{array}$ & DV \\
\hline 531 & Scully, T. Obesity. Nature.2014;508:S49. & Design \\
\hline 532 & Seal, N.,Broome, M. E. Prepregnancy Body Mass Index and Feeding Practices in Relation to Infants' Growth. J Nurse & Design \\
\hline
\end{tabular}




\begin{tabular}{|c|c|c|}
\hline & Pract.2013;9. & \\
\hline 533 & $\begin{array}{l}\text { Seksaria,S. A.,Sheth,M. K. Mass media as a means to bring about behavioral changes in infant and young child feeding practices } \\
\text { amongst tribal mothers of Chikhli taluka, Gujarat. Indian Journal of Public Health Research and Development.2015;6:113-118. }\end{array}$ & Design, IV \\
\hline 534 & $\begin{array}{l}\text { Sellen, D. W.,Thompson, A. L.,Hruschka, D. J.,Stein, A. D.,Martorell, R. Early determinants of non-exclusive breastfeeding } \\
\text { among Guatemalan infants. Adv Exp Med Biol.2004;554:299-301. }\end{array}$ & IV, DV \\
\hline 535 & $\begin{array}{l}\text { Senarath, U.,Godakandage, S. S.,Jayawickrama, H.,Siriwardena, I.,Dibley, M. J. Determinants of inappropriate complementary } \\
\text { feeding practices in young children in Sri Lanka: secondary data analysis of Demographic and Health Survey 2006-2007. Matern } \\
\text { Child Nutr.2012;8 Suppl 1:60-77. }\end{array}$ & Design,DV \\
\hline 536 & $\begin{array}{l}\text { Sethi, V.,Kashyap, S.,Seth, V. Effect of nutrition education of mothers on infant feeding practices. Indian J Pediatr.2003;70:463- } \\
6 .\end{array}$ & IV \\
\hline 537 & $\begin{array}{l}\text { Sezer,R. G.,Aydemir,G.,Akcan,A. B.,Bayoglu,D. S.,Guran,T.,Bozaykut,A. Effect of breastfeeding on serum zinc levels and growth } \\
\text { in healthy infants. Breastfeed Med.2013;8:159-63. }\end{array}$ & Design \\
\hline 538 & $\begin{array}{l}\text { Shaternikov,V. A.,Fateeva,E. M.,Chernikov,M. N. Protein nutrition in early infancy and subsequent periods: its effect on further } \\
\text { development. Bibl Nutr Dieta.1982:95-111. }\end{array}$ & Design \\
\hline 539 & $\begin{array}{l}\text { Shehadeh, N.,Weitzer-Kish, H.,Shamir, R.,Shihab, S.,Weiss, R. Impact of early postnatal weight gain and feeding patterns on } \\
\text { body mass index in adolescence. J Pediatr Endocrinol Metab.2008;21:9-15. }\end{array}$ & IV \\
\hline 540 & $\begin{array}{l}\text { Shi,L.,Zhang,J.,Wang,Y.,Caulfield,L. E.,Guyer,B. Effectiveness of an educational intervention on complementary feeding } \\
\text { practices and growth in rural China: a cluster randomised controlled trial. Public Health Nutr.2010;13:556-65. }\end{array}$ & IV \\
\hline 541 & $\begin{array}{l}\text { Shim, J. E.,Kim, J.,Mathai, R. A. Associations of infant feeding practices and picky eating behaviors of preschool children. J Am } \\
\text { Diet Assoc.2011;111:1363-8. }\end{array}$ & DV \\
\hline 542 & $\begin{array}{l}\text { Simell, O.,Niinikoski, H.,Viikari, J.,Rask-Nissila, L., Tammi, A., Ronnemaa, T. Cardiovascular disease risk factors in young children } \\
\text { in the STRIP baby project. Special Turku coronary Risk factor Intervention Project for children. Ann Med.1999;31 Suppl 1:55-61. }\end{array}$ & IV \\
\hline 543 & $\begin{array}{l}\text { Simondon, K. B.,Gartner, A.,Berger, J.,Cornu, A.,Massamba, J. P.,San Miguel, J. L.,Ly, C.,Missotte, I.,Simondon, F., Traissac, } \\
\text { P.,Delpeuch, F.,Maire, B. Effect of early, short-term supplementation on weight and linear growth of 4-7-mo-old infants in } \\
\text { developing countries: a four-country randomized trial. Am J Clin Nutr.1996;64:537-45. }\end{array}$ & IV,Country \\
\hline 544 & $\begin{array}{l}\text { Singhal A,Cole TJ,Fewtrell M,Kennedy K,Stephenson T,Elias-Jones A,Lucas A. Promotion of faster weight gain in infants born } \\
\text { small for gestational age: is there an adverse effect on later blood pressure?. Circulation.2007;115:213-20. }\end{array}$ & $\begin{array}{l}\text { Health } \\
\text { status, IV }\end{array}$ \\
\hline 545 & Singhal, A.,Lucas, A. Early origins of cardiovascular disease: is there a unifying hypothesis?. Lancet.2004;363:1642-5. & Design \\
\hline 546 & $\begin{array}{l}\text { Skau,J. K.,Touch,B.,Chhoun,C.,Chea,M.,Unni,U. S.,Makurat,J.,Filteau,S.,Wieringa,F. T.,Dijkhuizen,M. A.,Ritz,C.,Wells,J. } \\
\text { C.,Berger,J.,Friis,H.,Michaelsen,K. F.,Roos,N. Effects of animal source food and micronutrient fortification in complementary food } \\
\text { products on body composition, iron status, and linear growth: a randomized trial in Cambodia. Am J Clin Nutr.2015;101:742-51. }\end{array}$ & IV \\
\hline 547 & $\begin{array}{l}\text { Skledar, M. T.,Milosevic, M. Breastfeeding and time of complementary food introduction as predictors of obesity in children. Cent } \\
\text { Eur J Public Health.2015;23:26-31. }\end{array}$ & Design \\
\hline
\end{tabular}




\begin{tabular}{|c|c|c|}
\hline 548 & $\begin{array}{l}\text { Sloan, S., Gildea, A.,Stewart, M.,Sneddon, H.,Iwaniec, D. Early weaning is related to weight and rate of weight gain in infancy. } \\
\text { Child Care Health Dev.2008;34:59-64. }\end{array}$ & Design \\
\hline 549 & $\begin{array}{l}\text { Snijders, B. E.,Thijs, C.,van Ree, R.,van den Brandt, P. A. Age at first introduction of cow milk products and other food products } \\
\text { in relation to infant atopic manifestations in the first } 2 \text { years of life: the KOALA Birth Cohort Study. Pediatrics.2008;122:e115-22. }\end{array}$ & DV \\
\hline 550 & $\begin{array}{l}\text { Soto-Ramirez, N.,Karmaus, W.,Zhang, H.,Davis, S.,Agarwal, S.,Albergottie, A. Modes of infant feeding and the occurrence of } \\
\text { coughing/wheezing in the first year of life. J Hum Lact.2013;29:71-80. }\end{array}$ & IV, DV \\
\hline 551 & Sparks, J. W. Fetal growth and diet. Mead Johnson Symp Perinat Dev Med.1984:21-7. & Design \\
\hline 552 & $\begin{array}{l}\text { Specker BL,Beck A,Kalkwarf H,Ho M. Randomized trial of varying mineral intake on total body bone mineral accretion during the } \\
\text { first year of life. Pediatrics.1997;99:E12. }\end{array}$ & IV \\
\hline 553 & $\begin{array}{l}\text { Spence,A. C.,Campbell,K. J.,Crawford,D. A.,McNaughton,S. A.,Hesketh,K. D. Mediators of improved child diet quality following a } \\
\text { health promotion intervention: the Melbourne InFANT Program. Int J Behav Nutr Phys Act.2014;11:137. }\end{array}$ & IV, DV \\
\hline 554 & $\begin{array}{l}\text { Sreedhara, M. S.,Banapurmath, C. R. A study of nutritional status of infants in relation to their complementary feeding practices. } \\
\text { Current Pediatric Research.2014;18:39-41. }\end{array}$ & Design \\
\hline 555 & Stahl, M. D.,Guida, D. A. Slow weight gain in the breast-fed infant: management options. Pediatr Nurs.1984;10:117-20, 164. & Design \\
\hline 556 & Stang,J. Improving the eating patterns of infants and toddlers. J Am Diet Assoc.2006;106:S7-9. & Design \\
\hline 557 & $\begin{array}{l}\text { Stifter, C. A.,Anzman-Frasca, S.,Birch, L. L.,Voegtline, K. Parent use of food to soothe infant/toddler distress and child weight } \\
\text { status. An exploratory study. Appetite.2011;57:693-9. }\end{array}$ & Design, IV \\
\hline 558 & $\begin{array}{l}\text { Strbak, V.,Skultetyova, M.,Hromadova, M.,Randuskova, A.,Macho, L. Late effects of breast-feeding and early weaning: seven- } \\
\text { year prospective study in children. Endocr Regul.1991;25:53-7. }\end{array}$ & IV \\
\hline 559 & $\begin{array}{l}\text { Stuff, J. E.,Garza, C.,Boutte, C.,Fraley, J. K.,Smith, E. O.,Klein, E. R.,Nichols, B. L. Sources of variance in milk and caloric } \\
\text { intakes in breast-fed infants: implications for lactation study design and interpretation. Am J Clin Nutr.1986;43:361-6. }\end{array}$ & Design, IV \\
\hline 560 & Stuff, J. E.,Nichols, B. L. Nutrient intake and growth performance of older infants fed human milk. J Pediatr.1989;115:959-68. & DV \\
\hline 561 & $\begin{array}{l}\text { Stunkard, A. J.,Berkowitz, R. I.,Schoeller, D.,Maislin, G.,Stallings, V. A. Predictors of body size in the first } 2 \text { y of life: a high-risk } \\
\text { study of human obesity. Int J Obes Relat Metab Disord.2004;28:503-13. }\end{array}$ & IV \\
\hline 562 & $\begin{array}{l}\text { Stunkard, A. J.,Berkowitz, R. I.,Stallings, V. A.,Schoeller, D. A. Energy intake, not energy output, is a determinant of body size in } \\
\text { infants. Am J Clin Nutr.1999;69:524-30. }\end{array}$ & IV \\
\hline 563 & $\begin{array}{l}\text { Super, C. M.,Herrera, M. G.,Mora, J. O. Long-term effects of food supplementation and psychosocial intervention on the physical } \\
\text { growth of Colombian infants at risk of malnutrition. Child Dev.1990;61:29-49. }\end{array}$ & IV \\
\hline 564 & $\begin{array}{l}\text { Svahn,J. C.,Feldl,F.,Raiha,N. C.,Koletzko,B.,Axelsson,I. E. Different quantities and quality of fat in milk products given to young } \\
\text { children: effects on long chain polyunsaturated fatty acids and trans fatty acids in plasma. Acta Paediatr.2002;91:20-9. }\end{array}$ & DV \\
\hline 565 & $\begin{array}{l}\text { Taitz, L. S.,Lukmanji, Z. Alterations in feeding patterns and rates of weight gain in South Yorkshire infants, 1971-1977. Hum } \\
\text { Biol.1981;53:313-20. }\end{array}$ & IV, Date \\
\hline
\end{tabular}




\begin{tabular}{|c|c|c|}
\hline 566 & Taitz, L. Feeding children in the first year of life. Midwife Health Visitor \& Community Nurse.1990;26:81-84. & Design \\
\hline 567 & Tann, S. P.,Wheeler, E. F. Food intakes and growth of young Chinese children in London. Community Med.1980;2:20-4. & IV, DV \\
\hline 568 & $\begin{array}{l}\text { Tantracheewathorn,S.,Lohajaroensub,S. Incidence and risk factors of iron deficiency anemia in term infants. J Med Assoc } \\
\text { Thai.2005;88:45-51. }\end{array}$ & IV, DV \\
\hline 569 & $\begin{array}{l}\text { Tanzer F,Gumuser C. A study of the growth of } 200 \text { newborn babies for a period of } 6 \text { months according to the type of nutrition. } \\
\text { Ann Trop Paediatr.1989;9:54-8. }\end{array}$ & IV \\
\hline 570 & $\begin{array}{l}\text { Tarrant, M.,Fong, D. Y.,Wu, K. M.,Lee, I. L.,Wong, E. M.,Sham, A.,Lam, C., Dodgson, J. E. Breastfeeding and weaning practices } \\
\text { among Hong Kong mothers: a prospective study. BMC Pregnancy Childbirth.2010;10:27. }\end{array}$ & DV \\
\hline 571 & $\begin{array}{l}\text { Taveras EM,Blackburn K,Gillman MW,Haines J,McDonald J,Price S,Oken E. First steps for mommy and me: a pilot intervention } \\
\text { to improve nutrition and physical activity behaviors of postpartum mothers and their infants. Matern Child Health J.2011;15:1217- } \\
27 \text {. }\end{array}$ & IV \\
\hline 572 & $\begin{array}{l}\text { Tawia, S. Breastfeeding, brain structure and function, cognitive development and educational attainment. Breastfeed } \\
\text { Rev.2013;21:15-20. }\end{array}$ & Design \\
\hline 573 & Tawia, S. Childhood obesity and being breastfed. Breastfeed Rev.2013;21:42-8. & Design \\
\hline 574 & $\begin{array}{l}\text { Taylor,A.,Redworth,E. W.,Morgan,J. B. Influence of diet on iron, copper, and zinc status in children under } 24 \text { months of age. Biol } \\
\text { Trace Elem Res.2004;97:197-214. }\end{array}$ & DV \\
\hline 575 & $\begin{array}{l}\text { Thakur, R.,Singh, M. G.,Chaudhary, S.,Manuja, N. Effect of mode of delivery and feeding practices on acquisition of oral } \\
\text { Streptococcus mutans in infants. Int J Paediatr Dent.2012;22:197-202. }\end{array}$ & DV \\
\hline 576 & $\begin{array}{l}\text { Theron, M.,Amissah, A.,Kleynhans, I. C.,Albertse, E.,Maclntyre, U. E. Inadequate dietary intake is not the cause of stunting } \\
\text { amongst young children living in an informal settlement in Gauteng and rural Limpopo Province in South Africa: the NutriGro } \\
\text { study. Public Health Nutr.2007;10:379-89. }\end{array}$ & Design \\
\hline 577 & $\begin{array}{l}\text { Thomson, J. L.,Tussing-Humphreys, L. M.,Goodman, M. H. Delta Healthy Sprouts: a randomized comparative effectiveness trial } \\
\text { to promote maternal weight control and reduce childhood obesity in the Mississippi Delta. Contemp Clin Trials.2014;38:82-91. }\end{array}$ & Design, IV \\
\hline 578 & $\begin{array}{l}\text { Thorisdottir, B.,Gunnarsdottir, I.,Thorisdottir, A. V.,Palsson, G. I.,Halldorsson, T. I.,Thorsdottir, I. Nutrient intake in infancy and } \\
\text { body mass index at six years in two population-based cohorts recruited before and after revision of infant dietary } \\
\text { recommendations. Ann Nutr Metab.2013;63:145-51. }\end{array}$ & IV \\
\hline 579 & $\begin{array}{l}\text { Thorsdottir, I.,Gunnarsdottir, I.,Palsson, G. I. Birth weight, growth and feeding in infancy: relation to serum lipid concentration in } \\
\text { 12-month-old infants. Eur J Clin Nutr.2003;57:1479-85. }\end{array}$ & IV, DV \\
\hline 580 & $\begin{array}{l}\text { Timby, N.,Domellof, E.,Hernell, O.,Lonnerdal, B.,Domellof, M. Neurodevelopment, nutrition, and growth until } 12 \text { mo of age in } \\
\text { infants fed a low-energy, low-protein formula supplemented with bovine milk fat globule membranes: a randomized controlled } \\
\text { trial. Am J Clin Nutr.2014;99:860-8. }\end{array}$ & IV \\
\hline 581 & $\begin{array}{l}\text { Townsend, E.,Pitchford, N. J. Baby knows best? The impact of weaning style on food preferences and body mass index in early } \\
\text { childhood in a case-controlled sample. BMJ Open.2012;2:e000298. }\end{array}$ & IV \\
\hline
\end{tabular}




\begin{tabular}{|c|c|c|}
\hline 582 & $\begin{array}{l}\text { Tripathy, R.,Das, R. N.,Das, M. M.,Parija, A. C. Growth in the first year in children following IAP Policy on Infant Feeding. Indian } \\
\text { Pediatr.2000;37:1051-9. }\end{array}$ & IV \\
\hline 583 & $\begin{array}{l}\text { Tulldahl, J.,Pettersson, K.,Andersson, S. W.,Hulthen, L. Mode of infant feeding and achieved growth in adolescence: early } \\
\text { feeding patterns in relation to growth and body composition in adolescence. Obes Res.1999;7:431-7. }\end{array}$ & Design \\
\hline 584 & $\begin{array}{l}\text { Umer, A.,Hamilton, C.,Britton, C. M.,Mullett, M. D.,John, C.,Neal, W.,Lilly, C. L. Association between Breastfeeding and } \\
\text { Childhood Obesity: Analysis of a Linked Longitudinal Study of Rural Appalachian Fifth-Grade Children. Child Obes.2015;11:449- } \\
55 .\end{array}$ & IV \\
\hline 585 & $\begin{array}{l}\text { Unni, J. C.,Richard, J. Growth and morbidity of breast-fed and artificially-fed infants in urban south Indian families. J Trop } \\
\text { Pediatr.1988;34:179-81. }\end{array}$ & IV \\
\hline 586 & $\begin{array}{l}\text { Vail, B.,Prentice, P.,Dunger, D. B.,Hughes, I. A.,Acerini, C. L.,Ong, K. K. Age at Weaning and Infant Growth: Primary Analysis } \\
\text { and Systematic Review. Journal of Pediatrics.2015;167:317-324.e1. }\end{array}$ & Design \\
\hline 587 & Valman, H. B. The first year of life: feeding and feeding problems. Br Med J.1980;280:457-60. & Design \\
\hline 588 & $\begin{array}{l}\text { van der Willik, E. M.,Vrijkotte, T. G.,Altenburg, T. M.,Gademan, M. G.,Kist-van Holthe, J. Exclusively breastfed overweight infants } \\
\text { are at the same risk of childhood overweight as formula fed overweight infants. Arch Dis Child.2015;100:932-7. }\end{array}$ & IV \\
\hline 589 & $\begin{array}{l}\text { van Dijk, C. E.,Innis, S. M. Growth-curve standards and the assessment of early excess weight gain in infancy. } \\
\text { Pediatrics.2009;123:102-8. }\end{array}$ & IV, DV \\
\hline 590 & $\begin{array}{l}\text { van Eijsden, M.,Meijers, C. M.,Jansen, J. E.,de Kroon, M. L.,Vrijkotte, T. G. Cultural variation in early feeding pattern and } \\
\text { maternal perceptions of infant growth. Br J Nutr.2015;114:481-8. }\end{array}$ & DV \\
\hline 591 & $\begin{array}{l}\text { van Rheenen,P. F.,de Moor,L. T.,Eschbach,S.,Brabin,B. J. A cohort study of haemoglobin and zinc protoporphyrin levels in term } \\
\text { Zambian infants: effects of iron stores at birth, complementary food and placental malaria. Eur J Clin Nutr.2008;62:1379-87. }\end{array}$ & Country \\
\hline 592 & $\begin{array}{l}\text { van } t \text { Hof Msc, M. A. The influence of breastfeeding and complementary foods on growth until three years of age in the Euro- } \\
\text { Growth Study. Pediatrics.2000;106:1281a-1281. }\end{array}$ & Design \\
\hline 593 & $\begin{array}{l}\text { Vazir,S.,Engle,P.,Balakrishna,N.,Griffiths,P. L.,Johnson,S. L.,Creed-Kanashiro,H.,Fernandez Rao,S.,Shroff,M. R.,Bentley,M. E. } \\
\text { Cluster-randomized trial on complementary and responsive feeding education to caregivers found improved dietary intake, growth } \\
\text { and development among rural Indian toddlers. Matern Child Nutr.2013;9:99-117. }\end{array}$ & IV \\
\hline 594 & $\begin{array}{l}\text { Veena SR,Krishnaveni GV,Srinivasan K,Wills AK,Hill JC,Kurpad AV,Muthayya S,Karat SC,Nalinakshi M,Fall CH. Infant feeding } \\
\text { practice and childhood cognitive performance in South India. Arch Dis Child.2010;95:347-54. }\end{array}$ & Country \\
\hline 595 & $\begin{array}{l}\text { Veena, S. R.,Krishnaveni, G. V.,Wills, A. K.,Hill, J. C.,Karat, S. C.,Fall, C. H. Glucose tolerance and insulin resistance in Indian } \\
\text { children: relationship to infant feeding pattern. Diabetologia.2011;54:2533-7. }\end{array}$ & Country \\
\hline 596 & $\begin{array}{l}\text { Vehapoglu, A.,Yazici, M.,Demir, A. D.,Turkmen, S.,Nursoy, M.,Ozkaya, E. Early infant feeding practice and childhood obesity: the } \\
\text { relation of breast-feeding and timing of solid food introduction with childhood obesity. J Pediatr Endocrinol Metab.2014;27:1181- } \\
7 \text {. }\end{array}$ & Design \\
\hline 597 & $\begin{array}{l}\text { Venancio, S. I.,Saldiva, S. R.,Mondini, L.,Levy, R. B.,Escuder, M. M. Early interruption of exclusive breastfeeding and associated } \\
\text { factors, state of Sao Paulo, Brazil. J Hum Lact.2008;24:168-74. }\end{array}$ & $\begin{array}{l}\text { Design, } \\
\text { DV }\end{array}$ \\
\hline
\end{tabular}




\begin{tabular}{|c|c|c|}
\hline 598 & $\begin{array}{l}\text { Verd S,Barriuso L,Gich I,Gutierrez A,Nadal-Amat J,Carreras E. Risk of early breastfeeding cessation among symmetrical, small } \\
\text { for gestational age infants. Ann Hum Biol.2013;40:146-51. }\end{array}$ & $\begin{array}{l}\text { Health } \\
\text { status, DV }\end{array}$ \\
\hline 599 & $\begin{array}{l}\text { Victora, C. G.,Matijasevich, A.,Santos, I. S.,Barros, A. J.,Horta, B. L.,Barros, F. C. Breastfeeding and feeding patterns in three } \\
\text { birth cohorts in Southern Brazil: trends and differentials. Cad Saude Publica.2008;24 Suppl 3:S409-16. }\end{array}$ & IV, DV \\
\hline 600 & $\begin{array}{l}\text { Victora,C. G.,Vaughan,J. P.,Martines,J. C.,Barcelos,L. B. Is prolonged breast-feeding associated with malnutrition?. Am J Clin } \\
\text { Nutr.1984;39:307-14. }\end{array}$ & IV \\
\hline 601 & Villalpando, S. Feeding mode, infections, and anthropometric status in early childhood. Pediatrics.2000;106:1282-3. & Design \\
\hline 602 & $\begin{array}{l}\text { Virtanen, S. M.,Laara, E.,Hypponen, E.,Reijonen, H.,Rasanen, L.,Aro, A.,Knip, M.,llonen, J.,Akerblom, H. K. Cow's milk } \\
\text { consumption, HLA-DQB1 genotype, and type } 1 \text { diabetes: a nested case-control study of siblings of children with diabetes. } \\
\text { Childhood diabetes in Finland study group. Diabetes.2000;49:912-7. }\end{array}$ & DV \\
\hline 603 & $\begin{array}{l}\text { Virtanen, S. M.,Rasanen, L.,Ylonen, K.,Aro, A.,Clayton, D.,Langholz, B.,Pitkaniemi, J.,Savilahti, E.,Lounamaa, R.,Tuomilehto, } \\
\text { J.,et al.,. Early introduction of dairy products associated with increased risk of IDDM in Finnish children. The Childhood in } \\
\text { Diabetes in Finland Study Group. Diabetes.1993;42:1786-90. }\end{array}$ & DV \\
\hline 604 & $\begin{array}{l}\text { Vobecky,J. S.,Vobecky,J.,Shapcott,D.,Demers,P. P. Nutrient intake patterns and nutritional status with regard to relative weight in } \\
\text { early infancy. Am J Clin Nutr.1983;38:730-8. }\end{array}$ & IV \\
\hline 605 & $\begin{array}{l}\text { Wandel, M.,Fagerli, R. Aa,Olsen, P. T.,Borch-lohnsen, B.,Ek, J. Iron status and weaning practices among Norwegian and } \\
\text { immigrant infants. Nutrition Research.1996;16:251-265. }\end{array}$ & Design \\
\hline 606 & $\begin{array}{l}\text { Wang RJ,Trehan I,LaGrone LN,Weisz AJ,Thakwalakwa CM,Maleta KM,Manary MJ. Investigation of food acceptability and } \\
\text { feeding practices for lipid nutrient supplements and blended flours used to treat moderate malnutrition. J Nutr Educ } \\
\text { Behav.2013;45:258-63. }\end{array}$ & $\begin{array}{l}\text { Health } \\
\text { status, } \\
\text { Country }\end{array}$ \\
\hline 607 & $\begin{array}{l}\text { Watt, R. G.,Tull, K. I.,Hardy, R.,Wiggins, M.,Kelly, Y.,Molloy, B.,Dowler, E.,Apps, J.,McGlone, P. Effectiveness of a social support } \\
\text { intervention on infant feeding practices: randomised controlled trial. J Epidemiol Community Health.2009;63:156-62. }\end{array}$ & IV, DV \\
\hline 608 & $\begin{array}{l}\text { Weber, M.,Grote, V.,Closa-Monasterolo, R.,Escribano, J.,Langhendries, J. P.,Dain, E.,Giovannini, M.,Verduci, E.,Gruszfeld, } \\
\text { D.,Socha, P.,Koletzko, B. Lower protein content in infant formula reduces BMl and obesity risk at school age: follow-up of a } \\
\text { randomized trial. Am J Clin Nutr.2014;99:1041-51. }\end{array}$ & IV \\
\hline 609 & $\begin{array}{l}\text { Weijs, P. J.,Kool, L. M.,van Baar, N. M.,van der Zee, S. C. High beverage sugar as well as high animal protein intake at infancy } \\
\text { may increase overweight risk at } 8 \text { years: a prospective longitudinal pilot study. Nutr J.2011;10:95. }\end{array}$ & IV \\
\hline 610 & $\begin{array}{l}\text { Wen, L. M.,Baur, L. A.,Simpson, J. M.,Xu, H.,Hayes, A. J.,Hardy, L. L.,Williams, M.,Rissel, C. Sustainability of Effects of an Early } \\
\text { Childhood Obesity Prevention Trial Over Time: A Further 3-Year Follow-up of the Healthy Beginnings Trial. JAMA } \\
\text { Pediatr.2015;169:543-51. }\end{array}$ & IV \\
\hline 611 & $\begin{array}{l}\text { West, C. E.,Hernell, O.,Andersson, Y.,Sjostedt, M.,Hammarstrom, M. L. Probiotic effects on T-cell maturation in infants during } \\
\text { weaning. Clin Exp Allergy.2012;42:540-9. }\end{array}$ & IV, DV \\
\hline 612 & Westphal, R.,Phillips, G.,Irwig, L. M. Infant care and feeding in an urban black population. S Afr Med J.1981;60:778-81. & DV, Date \\
\hline
\end{tabular}




\begin{tabular}{|c|c|c|}
\hline 613 & $\begin{array}{l}\text { Weyermann, M.,Rothenbacher, D.,Brenner, H. Duration of breastfeeding and risk of overweight in childhood: a prospective birth } \\
\text { cohort study from Germany. Int J Obes (Lond).2006;30:1281-7. }\end{array}$ & IV \\
\hline 614 & $\begin{array}{l}\text { Wharf,S. G.,Fox,T. E.,Fairweather-Tait,S. J.,Cook,J. D. Factors affecting iron stores in infants 4-18 months of age. Eur J Clin } \\
\text { Nutr.1997;51:504-9. }\end{array}$ & Design \\
\hline 615 & $\begin{array}{l}\text { Whitehead, R. G.,Paul, A. A.,Ahmed, E. A. Weaning practices in the United Kingdom and variations in anthropometric } \\
\text { development. Acta Paediatr Scand Suppl.1986;323:14-23. }\end{array}$ & Design, IV \\
\hline 616 & Whitehead, R. G.,Paul, A. A. Infant growth and human milk requirements. A fresh approach. Lancet.1981;2:161-3. & IV \\
\hline 617 & $\begin{array}{l}\text { Whitten, C. F.,Stewart, R. A. The effect of dietary sodium in infancy on blood pressure and related factors. Studies of infants fed } \\
\text { salted and unsalted diets for five months at eight months and eight years of age. Acta Paediatr Scand Suppl.1980;279:1-17. }\end{array}$ & IV \\
\hline 618 & $\begin{array}{l}\text { Wiberger, M.,Eiben, G.,Lissner, L.,Mehlig, K.,Papoutsou, S.,Hunsberger, M. Children consuming milk cereal drink are at } \\
\text { increased risk for overweight: The IDEFICS Sweden study, on behalf of the IDEFICS Consortium. Scand J Public } \\
\text { Health.2014;42:518-24. }\end{array}$ & Design \\
\hline 619 & $\begin{array}{l}\text { Wigg, N. R.,Tong, S.,McMichael, A. J.,Baghurst, P. A.,Vimpani, G.,Roberts, R. Does breastfeeding at six months predict cognitive } \\
\text { development?. Aust N Z J Public Health.1998;22:232-6. }\end{array}$ & IV \\
\hline 620 & $\begin{array}{l}\text { Wijga,A.,Vyas,U.,Vyas,A.,Sharma,V.,Pandya,N.,Nabarro,D. Feeding, illness and nutritional status of young children in rural } \\
\text { Gujarat. Hum Nutr Clin Nutr.1983;37:255-69. }\end{array}$ & Design, IV \\
\hline 621 & $\begin{array}{l}\text { Williams, D. M.,Martin, R. M.,Davey Smith, G.,Alberti, K. G.,Ben-Shlomo, Y.,McCarthy, A. Associations of infant nutrition with } \\
\text { insulin resistance measures in early adulthood: evidence from the Barry-Caerphilly Growth (BCG) study. PLoS } \\
\text { One.2012;7:e34161. }\end{array}$ & IV, DV \\
\hline 622 & $\begin{array}{l}\text { Williams, J.,Wolff, A.,Daly, A.,MacDonald, A.,Aukett, A.,Booth, I. W. Iron supplemented formula milk related to reduction in } \\
\text { psychomotor decline in infants from inner city areas: randomised study. Bmj.1999;318:693-7. }\end{array}$ & IV \\
\hline 623 & Winick, M. The role of early nutrition in subsequent development and optimal future health. Bull N Y Acad Med.1989;65:1020-5. & Design \\
\hline 624 & Winkelstein,M. L. Overfeeding in infancy: the early introduction of solid foods. Pediatr Nurs.1984;10:205-8, 236. & Design \\
\hline 625 & $\begin{array}{l}\text { Wölfle, J. Growth and puberty in German children: is there still a positive secular trend? In reply..Consumption of milk as a vital } \\
\text { factor in growth development. Melnik B, Dtsch Arzetebl 2009, volume 206. Deutsches Aerzteblatt International.2009;106:656- } \\
656 .\end{array}$ & Design \\
\hline 626 & $\begin{array}{l}\text { Wright, C. M.,Parkinson, K. N.,Drewett, R. F. Why are babies weaned early? Data from a prospective population based cohort } \\
\text { study. Arch Dis Child.2004;89:813-6. }\end{array}$ & DV \\
\hline 627 & $\begin{array}{l}\text { Wright, C. M.,Parkinson, K.,Scott, J. Breast-feeding in a UK urban context: who breast-feeds, for how long and does it matter?. } \\
\text { Public Health Nutr.2006;9:686-91. }\end{array}$ & IV \\
\hline 628 & $\begin{array}{l}\text { Wright, M. J.,Bentley, M. E.,Mendez, M. A.,Adair, L. S. The interactive association of dietary diversity scores and breast-feeding } \\
\text { status with weight and length in Filipino infants aged 6-24 months. Public Health Nutr.2015;18:1762-73. }\end{array}$ & Country \\
\hline
\end{tabular}




\begin{tabular}{|c|c|c|}
\hline 629 & $\begin{array}{l}\text { Yew, K. S.,Webber, B.,Hodges, J.,Carter, N. J. Clinical inquiries: are there any known health risks to early introduction of solids to } \\
\text { an infant's diet?. J Fam Pract.2009;58:219-20. }\end{array}$ & Design \\
\hline 630 & $\begin{array}{l}\text { Young RJ,Antonson DL,Ferguson PW,Murray ND,Merkel K,Moore TE. Neonatal and infant feeding: effect on bone density at } 4 \\
\text { years. J Pediatr Gastroenterol Nutr.2005;41:88-93. }\end{array}$ & Design \\
\hline 631 & $\begin{array}{l}\text { Yousafzai, A. K.,Rasheed, M. A.,Rizvi, A.,Armstrong, R.,Bhutta, Z. A. Effect of integrated responsive stimulation and nutrition } \\
\text { interventions in the Lady Health Worker programme in Pakistan on child development, growth, and health outcomes: a cluster- } \\
\text { randomised factorial effectiveness trial. Lancet.2014;384:1282-93. }\end{array}$ & Country \\
\hline 632 & $\begin{array}{l}\text { Ystrom, E. Breastfeeding cessation and symptoms of anxiety and depression: a longitudinal cohort study. BMC Pregnancy } \\
\text { Childbirth.2012;12:36. }\end{array}$ & IV, DV \\
\hline 633 & $\begin{array}{l}\text { Zadik Z,Borondukov E,Zung A,Reifen R. Adult height and weight of breast-fed and bottle-fed Israeli infants. J Pediatr } \\
\text { Gastroenterol Nutr.2003;37:462-7. }\end{array}$ & IV \\
\hline 634 & $\begin{array}{l}\text { Zaman, S.,Jalil, F.,Saleemi, M. A.,Mellander, L.,Ashraf, R. N.,Hanson, L. A. Changes in feeding patterns affect growth in children } \\
0-24 \text { months of age living in socioeconomically different areas of Lahore, Pakistan. Adv Exp Med Biol.2002;503:49-56. }\end{array}$ & Country \\
\hline 635 & $\begin{array}{l}\text { Zaman,S.,Ashraf,R. N.,Martines,J. Training in complementary feeding counselling of healthcare workers and its influence on } \\
\text { maternal behaviours and child growth: a cluster-randomized controlled trial in Lahore, Pakistan. J Health Popul } \\
\text { Nutr.2008;26:210-22. }\end{array}$ & $\begin{array}{l}\text { IV, } \\
\text { Country }\end{array}$ \\
\hline 636 & $\begin{array}{l}\text { Zavaleta,N.,Kvistgaard,A. S.,Graverholt,G.,Respicio,G.,Guija,H.,Valencia,N.,Lonnerdal,B. Efficacy of an MFGM-enriched } \\
\text { complementary food in diarrhea, anemia, and micronutrient status in infants. J Pediatr Gastroenterol Nutr.2011;53:561-8. }\end{array}$ & DV \\
\hline 637 & $\begin{array}{l}\text { Zhang,J.,Shi,L.,Chen,D. F.,Wang,J.,Wang,Y. Effectiveness of an educational intervention to improve child feeding practices and } \\
\text { growth in rural China: updated results at } 18 \text { months of age. Matern Child Nutr.2013;9:118-29. }\end{array}$ & IV \\
\hline 638 & $\begin{array}{l}\text { Zhu, B.,Zhang, J.,Qiu, L.,Binns, C.,Shao, J.,Zhao, Y.,Zhao, Z. Breastfeeding Rates and Growth Charts--the Zhejiang Infant } \\
\text { Feeding Trial. Int J Environ Res Public Health.2015;12:7337-47. }\end{array}$ & IV, DV \\
\hline 639 & $\begin{array}{l}\text { Ziegler, E. E.,Fields, D. A.,Chernausek, S. D.,Steenhout, P.,Grathwohl, D.,Jeter, J. M.,Nelson, S. E.,Haschke, F. Adequacy of } \\
\text { Infant Formula With Protein Content of } 1.6 \mathrm{~g} / 100 \mathrm{kcal} \text { for Infants Between } 3 \text { and } 12 \text { Months. J Pediatr Gastroenterol } \\
\text { Nutr.2015;61:596-603. }\end{array}$ & IV \\
\hline 640 & $\begin{array}{l}\text { Ziegler,E. E.,Fomon,S. J.,Nelson,S. E.,Rebouche,C. J.,Edwards,B. B.,Rogers,R. R., Lehman,L. J. Cow milk feeding in infancy: } \\
\text { further observations on blood loss from the gastrointestinal tract. J Pediatr.1990;116:11-8. }\end{array}$ & IV, DV \\
\hline 641 & $\begin{array}{l}\text { Zive, M. M.,McKay, H.,Frank-Spohrer, G. C.,Broyles, S. L.,Nelson, J. A.,Nader, P. R. Infant-feeding practices and adiposity in 4-y- } \\
\text { old Anglo- and Mexican-Americans. Am J Clin Nutr.1992;55:1104-8. }\end{array}$ & Design \\
\hline 642 & $\begin{array}{l}\text { Zutavern, A.,Brockow, I.,Schaaf, B.,von Berg, A.,Diez, U.,Borte, M.,Kraemer, U.,Herbarth, O.,Behrendt, H.,Wichmann, H. } \\
\text { E.,Heinrich, J. Timing of solid food introduction in relation to eczema, asthma, allergic rhinitis, and food and inhalant sensitization } \\
\text { at the age of } 6 \text { years: results from the prospective birth cohort study LISA. Pediatrics.2008;121:e44-52. }\end{array}$ & DV \\
\hline
\end{tabular}


${ }^{1}$ Abbreviations: DV- Dependent variable; IV- Independent variable/exposure/intervention 
